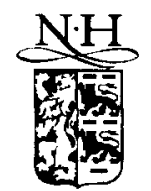

ELSEVIER

\title{
Gravitational fields near space-like and null infinity
}

\author{
Helmut Friedrich ${ }^{1}$ \\ Albert-Einstein-Institut, Max-Planck-Institut für Gravitationsphysik. Schlaatzweg I. \\ 14473 Potsdam, Germany
}

Received 19 December 1996

\begin{abstract}
Near space-like infinity an initial value problem for the conformal Einstein equations is formulated such that: (i) the data and equations are regular, (ii) space-like and null infinity have a finite representation, with their structure and location known a priori, and (iii) the setting relies entirely on general properties of conformal structures.

A first analysis of this problem shows that the solutions develop in general a certain type of logarithmic singularity at the set where null infinity touches space-like infinity. These singularities form an intrinsic part of the solutions' conformal structure. Conditions on the free initial data near space-like infinity are derived which ensure that for solutions developing from these data singularities of this type cannot occur.
\end{abstract}

Suhj. Class: General relativity

1991 MSC: 83C05

Keywords: Initial value problem; Einstein equations

\section{Introduction}

The conformal Einstein equations, obtained in [7,8] and elaborated in later papers, provide a highly efficient tool in analysing solutions to Einstein's vacuum field equation. In the case of non-vanishing cosmological constant they enable us to demonstrate for Einstein's equation the existence of semi-global and global solutions, to gain perfect control on the asymptotic structure of the latter, and to derive a wealth of important information on the solutions. In the case of Einstein's vacuum field equations with vanishing cosmological constant the conformal field equations have been used to show the existence of solutions

\footnotetext{
'Fax: 49(331) 275 37-98; e-mail: hef@ aei-potsdam.mpg.de.
} 
possessing a smooth asymptotic structure either in the past or in the future. These results are based on the use of hyperboloidal initial data which presuppose asymptotic smoothness on the initial hypersurface.

In all these cases Penrose's suggestion $[19,20]$ that the asymptotic behaviour of solutions to Einstein's equation can he characterized in terms of the conformal structure has been confirmed (cf. [13] for a recent survey and relevant references). It remained to be shown that smooth hyperboloidal vacuum data can arise by Einstein evolution from asymptotically flat standard Cauchy data.

Analysing solutions to the conformal field equations in the region where "null infinity touches space-like infinity" appeared to be impossibly difficult. In the model case of Minkowski space $\left(\mathbb{R}^{4}, \tilde{\eta}=\eta_{\mu \nu} \mathrm{d} x^{\mu} \mathrm{d} x^{\nu}\right)$ with metric coefficients $\eta_{\mu \nu}=\operatorname{diag}(1,-1$, $-1,-1)$ this region is obviously contained in the domain $D \equiv\left\{x_{\mu} x^{\mu}<0\right\}$. The coordinate transformation $x^{\mu} \rightarrow z^{\mu}=-\left(x_{\lambda} x^{\lambda}\right)^{-1} x^{\mu}$, which maps $D$ onto itself, renders the metric in the form $\tilde{\eta}=\left(z_{\lambda} z^{\lambda}\right)^{-2} \eta_{\mu \nu} \mathrm{d} z^{\mu} \mathrm{d} z^{\nu}$ and regions where the quantities $\left|z^{\mu}\right|$ are small represent neighbourhoods of space-like infinity. Rescaling $\tilde{\eta}$ with the conformal factor $\Omega=-z_{\lambda} z^{\lambda}$ we obtain the "conformal metric" $\eta \equiv \Omega^{2} \tilde{\eta}=\eta_{\mu \nu} \mathrm{d} z^{\mu} \mathrm{d} z^{\nu}$, which extends smoothly through the boundary $\partial D$ of $D$ in $\mathbb{R}^{4}$. We denote its extension to $D \cup \partial D$ again by $\eta$ and the smoothly extended conformal factor again by $\Omega$.

The set $\partial D$ decomposes into the sets scri ${ }^{+}=\left\{z^{\mu} \in \mathbb{R}^{4} \mid z^{0}>0, z_{\mu} z^{\mu}=0\right\}$, scri ${ }^{-}=$ $\left\{z^{\mu} \in \mathbb{R}^{4} \mid z^{0}<0, z_{\mu} z^{\mu}=0\right\}$, and the point $i^{0}=\left\{z^{\mu}=0\right\}$. The hypersurface scri ${ }^{+}$ ( $\left(\mathrm{scri}^{-}\right.$), which is null for the metric $\eta$, can be thought of as being generated by endpoints of future (past) directed null geodesics and thus represents part of future (past) null infinity for Minkowski space. Since all space-like geodesics on Minkowski space run ultimately into $i^{0}$, this point represents space-like infinity. In the extended space-time the hypersurfaces $\mathrm{scri}^{ \pm}$form the null cone through the point $i^{0}$. Notice that $\Omega$ vanishes quadratically at $i^{0}$ while it vanishes only linearly on $\mathrm{scri}^{ \pm}$.

Any Cauchy hypersurface $\tilde{S}$ of Minkowski space approaches $i^{0}$. If this point is added to $\tilde{S}$, the resulting set $S$ inherits a topology which makes it a compact manifold homeomorphic to $S^{3}$ and the point $i^{0}$ represents space-like infinity for the initial data induced on $\tilde{S}$. We can assume $\tilde{S}$ to be chosen such that $S$ acquires a smooth differentiable structure. The process of "conformal compactification" which embeds $\tilde{S}$ into $S$ and maps the Minkowskian initial data on $\tilde{S}$ onto conformally related fields can, of course, also be described intrinsically in terms of $\tilde{S}$ and the given data. The conformal factor on $S$ will then be positive on $\tilde{S}$, vanish quadratically at $i$, and have non-degenerate Hessian there. It will be convenient to distinguish between the notion of space-like infinity which refers to the four-dimensional space and the notion of space-like infinity which refers to a three-dimensional Cauchy hypersurface. We shall denote the former hy the symbol $i^{0}$ and the latter by $i$.

Consider now a space-like, asymptotically flat initial data set $\left(\tilde{S}, \tilde{h}_{\mu v}, \tilde{\chi}_{\mu \nu}\right)$ for the vacuum field equation, where $\tilde{h}_{\mu \nu}$ is a (negative definite) metric and $\tilde{\chi}_{\mu \nu}$ represents the second fundamental form. We may ask whether the solution determined by these data admits smooth asymptotic structures like those found above for Minkowski space.

If this were the case, the sets scri ${ }^{ \pm}$would, as a consequence of the field equations, necessarily be null hypersurfaces for the extended metric. The schematic pictures used to 
illustrate causal relationships in space-times suggest that for our solution space-like infinity should again be depicted as a point $i^{0}$. But the precise relationship between space-like and null infinity can, of course, not be inferred from schematic causal pictures and it appears extremely difficult to obtain any information about it by using the field equations. The source of this difficulty can be found directly in the behaviour of the initial data near spacelike infinity. The situation differs in general drastically from that observed in the case of Minkowski space.

We perform a conformal compactification $\tilde{S} \rightarrow S=\tilde{S} \cup\{i\}$ and an associated rescaling of the data as outlined above. It turns out that even under the most fortunate assumptions, i.e. if we had chosen data such that $S$ and the conformally rescaled data were smooth near $i$, the conformal Weyl tensor of the time evolution determined by the data would diverge at $i$ unless the ADM mass of the initial data vanished.

Thus we find in general the following property of the conformal structure. At its lowest level, at which it determines the causal relations, it suggests representing space-like infinity by a point. However, at a higher level such a representation will be too narrow for ficlds associated with the conformal structure to be well behaved.

For this reason it has been surmised occasionally that it does not suffice for the description of space-like infinity to consider exclusively the conformal structure. It has been suggested that other structures, e.g. the projective structure, should be taken into account as well. The singular behaviour of the conformal Weyl tensor makes the application of the conformal field equations quite difficult, the more so since one of the basic variables in these equations is the rescaled conformal Weyl tensor, which diverges even more strongly at $i$ (cf. (2.38) and (2.39)).

It will be shown in this article that it is possible to formulate for the conformal field equations an initial value problem near space-like infinity with the following properties. The data and the equations are regular. The relationship between space-like and null infinity and their location are known a priori. The form of the problem allows us to analyse the fields near space-like and null infinity in a finite picture. The setting relies entirely on general properties of conformal structures.

To explore the nature and demonstrate the efficiency of the setting we give a first analysis of the solutions near space-like infinity. It is shown that in general solutions develop a certain type of logarithmic singularity at the "set where null infinity touches space-like infinity". Since our setting is based entirely on general conformal properties, we can conclude that these singularities form an intrinsic part of the solutions' conformal structure. The origin of these singularities can be traced back to properties of the initial data. We derive (mild) conditions on the initial data near space-like infinity which ensure that singularities of this type cannot occur.

These investigations require a very detailed analysis of the structure of the data and the inter-relationships of the propagation equations, the constraint equations, and the geometry of the solution. It is only for this reason that we restrict the discussion in this article to time-symmetric initial data. The conformal properties we use to set up the initial value problem and the resulting form of the conformal field equations are independent of any such assumption. Since the constraint equations pose only weak restrictions on the regularity of 
the data at space-like infinity, we have, of course, to impose smoothness requirements there. To shorten the arguments we assume the data to be in fact analytic at space-like infinity. Results analogous to the ones derived here can, at the expense of complicated and lengthy recursion arguments, be obtained also for weaker differentiability assumptions.

To arrive at our initial value problem we have to make full use of the conformal structure. Besides conformal rescalings we need to use not just Levi-Civita connections but also conformal connections, i.e. torsion free connections for which parallel transport maps frames which are conformal for the given metric again onto such frames.

The natural place for such a discussion is the theory of normal conformal Cartan connections. It has been used in [12] to obtain the general conformal representation of the Einstein equation and the newly acquired conformal freedom is exploited to introduce gauge conditions for the conformal Einstein equations which are based on conformal geodesics. Certain properties of conformal geodesics derived in [12] are basic for the fact that we obtain a regular problem and that we can control the (finite) location of null infinity in terms of the initial data and some free function.

The final picture of the initial value problem near space-like infinity which we obtain here is different from any representation of space-like infinity considered before. The initial manifold $S$ is replaced by a compact manifold $\bar{S}$ with boundary by blowing up the point $i$ to a 2 -sphere which we denote by $I^{0}$. We thus write $\tilde{S}=\bar{S} \backslash I^{0}$. Close to $I^{0}$ we introduce a "radial" coordinate $\rho$ on $\vec{S}$ which vanishes on $I^{0}$ and is positive elsewhere. Near $I^{0}$ the manifold on which the solution is to be constructed is, in a suitable gauge, of the form $\bar{M}=\{(\tau, q) \in \mathbb{R} \times \bar{S} \| \tau \mid \leq 1+\rho(q)\}$, where we identify the initial data hypersurface $\bar{S}$ with $\{0\} \times \bar{S}$. Space-like infinity, thought of before as the point $i^{0}$, is now represented by the cylinder $I=\{|\tau|<1, \rho=0\}$.

Close to $I$ the hypersurfaces $\mathcal{I}^{ \pm}=\{\tau= \pm(1+\rho(q)), q \in \tilde{S}\}$ will represent null infinity for the solution, if the latter extends smoothly far enough into the future and the past. They "touch" $I$ at the sets $I^{ \pm}=\{\tau= \pm 1, \rho=0\}$. The curves $\{|\tau| \leq 1+\rho(q)\}$, with $q \in \tilde{S}$ fixed, are by construction conformal geodesics with natural parameter $\tau$.

In this setting the conformal Cauchy data on $\tilde{S}$ extend smoothly to $I^{0}$ and the propagation equations implied by the conformal field equations are symmetric hyperbolic near $\bar{S}$. Seen from the four-dimensional geometry, the set $I$ arises partly from a coordinate representation which in the radial direction is not adapted to the metric relations. For the conformal field equations the set $I$ is a regular hypersurface. Near $\vec{S}$ the existence of a smooth solution to our initial value problem follows from known results on symmetric hyperbolic systems.

The set $I$ is of a very special nature though. This hypersurface is "totally characteristic" in the sense that the symmetric hyperholic system of propagation equations reduces completely to an interior system on $I$. This allows us to determine all fields on $I$ from the data on $I^{0}$. As to be expected, no boundary values can be prescribed on $I$.

Moreover, by taking formal derivatives of the equations with respect to $\rho$, we get at any order transport equations on $I$ for the derivatives of the unknown fields. These derivatives on $I$ are determined, apart from calculational complexities, in this article. They define power series on $I$ which are convergent near $I^{0}$. 
At the sets $I^{ \pm}$the total characteristic $I$ is approached in $\bar{M}$ transversely by the sets $\mathcal{I}^{ \pm}$ which are necessarily null hypersurfaces and thus also characteristic. Therefore, a degeneracy of the propagation equations occurs at $I^{ \pm}$which entails a degeneracy of the transport equations when the sets $I^{ \pm}$are approached on $I$. An analysis of the transport equations, which can be used to discuss the fields near $I$ to any desired degree of precision, allows us to identify a certain class of logarithmic singularities which develop at $I^{ \pm}$. By the transport equations the occurrence of these singularities is related to properties of the initial data. For a certain type of expansion near $I^{0}$ of the free data on $\bar{S}$ those terms which generate the singularities can be identified and we obtain a precise criterion under which circumstances the singularities are avoided.

Whether the necessary conditions for regularity obtained by us are also sufficient to ensure the smoothness of the structure at null infinity will be investigated elsewhere. For us it is important that for the first time a setting is provided in which such questions can be analysed. It opens the door to a number of further investigations (cf. Section 9). The results of this article clearly demonstrate its efficiency. The final analysis should tell us under which circumstances Penrose's idea of asymptotic flatness is feasible. Whether these conditions will be realized for a sufficiently general class of solutions or not, in any case we will gain further insights into the structure of the equations, obtain thus important practical tools for the numerical construction of solutions, and clarify conceptual problems associated with the idea of an "isolated gravitating system" (cr. [13] for a discussion of some of the conceptual issues which arise in this context).

\section{Asymptotically flat initial data}

\subsection{General considerations}

We want to construct asymptotically flat solutions $(\tilde{M}, \tilde{g})$ of signature $(+,-,-,-$, to Einstein's equation from smooth data on some three-dimensional manifold $\tilde{S}$. The construction is to be arranged such that we may think of $\tilde{S}$ as being identified diffeomorphically with a smoothly embedded space-like Cauchy hypersurface of $(\tilde{M}, \tilde{g})$ and such that the data given on $\tilde{S}$ coincide with certain data implied on $\tilde{S}$ by $\tilde{g}$ and possibly some other fields. We shall need to investigate the behaviour of the solutions near space-like infinity in some detail. The inclusion of long range source fields would render the analysis quite complicated. On the other hand, when sources of compact support (e.g. fluid balls) are considered, the fields behave near space-like infinity as in the pure vacuum case. Therefore, we shall consider solutions to Einstein's vacuum field equation

$$
\operatorname{Ric}(\tilde{g})=0 \text {. }
$$

The relevant data on $\tilde{S}$ are then given by a metric $\tilde{h}_{\alpha \beta}$ of signature $(-,-,-)$ and a symmetric tensor field $\tilde{\chi}_{\alpha \beta}$, fields which represent the first and the second fundamental form induced by the metric $\tilde{g}$ on $\tilde{S}$, respectively (cf. [5]). Eq. (2.1) entails the constraint equations 


$$
\tilde{D}^{\alpha} \tilde{\chi}_{\alpha \beta}-\tilde{D}_{\beta} \tilde{\chi}^{\alpha}{ }_{\alpha}=0, \quad \tilde{r}-\left(\tilde{\chi}^{\alpha}{ }_{\alpha}\right)^{2}+\tilde{\chi}^{\alpha \beta} \tilde{\chi}_{\alpha \beta}=0,
$$

where $\tilde{D}$ denotes the Levi-Civita connection and $\tilde{r}$ the Ricci scalar of the metric $\tilde{h}$.

Since our goal is to understand the behaviour of far fields of isolated gravitating systems we shall require the data set $\left(\tilde{S}, \tilde{h}_{\alpha \beta}, \tilde{\chi}_{\alpha \beta}\right)$ to be "asymptotically flat" in the following sense. We assume that $(\tilde{S}, \tilde{h})$ be "asymptotically Euclidean", i.e. that there exists a compact subset of $\tilde{S}$ such that its complement is the union of disjoint subsets $\tilde{S}_{k}, k=1,2, \ldots, K$, of $\tilde{S}$, the "asymptotically flat ends", each of which can be identificd diffeomorphically with $\left\{y^{\alpha} \in\right.$ $\left.\mathbb{R}^{3}|| y \mid \equiv\left(\sum_{\alpha=1}^{3} y^{\alpha 2}\right)^{1 / 2}>r_{0}\right\}$ where $r_{0}$ is some positive real number. It is assumed that in the coordinates $y^{\alpha}$ introduced by this identification on $\tilde{S}_{k}$ the metric coefficients satisfy

$$
\tilde{h}_{\alpha \beta}=-\left(1+\frac{2 m_{k}}{|y|}\right) \delta_{\alpha \beta}+\mathrm{O}\left(\frac{1}{|y|^{2}}\right) \text { as }|y| \rightarrow \infty, m_{k}=\text { const. }
$$

A corresponding fall-off condition, namely

$$
\tilde{\chi}_{\alpha \beta}=\mathrm{O}\left(\frac{1}{|y|^{2}}\right), \quad \text { as }|y| \rightarrow \infty,
$$

is imposed on the second fundamental form.

In the context of Cauchy problems for Einstein's equations more general notions of asymptotical flatness have been considered (cf. [3-6] and the references given there). As an example we quote [6]. Here data are constructed which satisfy in standard coordinates $y^{\alpha}$ on $\mathbb{R}^{3}$

$$
\tilde{h}_{\alpha \beta}+\delta_{\alpha \beta} \in H_{s, \delta}\left(\mathbb{R}^{3}\right), \quad \tilde{\chi}_{\alpha \beta} \in H_{s, \delta+1}\left(\mathbb{R}^{3}\right), \quad s \geq 4, \delta>-\frac{3}{2} .
$$

The weighted Sobolev spaces $H_{s, \delta}\left(\mathbb{R}^{3}\right)$ used here are defined as sets of (measurable etc.) complex functions $u$ on $\mathbb{R}^{3}$ for which

$$
|u|_{s, \delta}=\left(\sum_{|\alpha|<s} \int\left(1+|y|^{2}\right)^{\delta+|\alpha|}\left|D^{\alpha} u\right|^{2} \mathrm{~d} y\right)^{1 / 2}<\infty .
$$

Data satisfying (2.3) and (2.4) are special cases of data which are characterized in terms of analogues on $\tilde{S}$ of the weighted Sobolev spaces $H_{s, \delta}\left(\mathbb{R}^{3}\right)$.

While conditions like (2.5) specify the smoothness of fields in a precise way in finite regions of the initial hypersurface, they leave considerable freedom for undesirable "nonsmoothness at space-like infinity". The detailed behaviour of the data near space-like infinity will most likely affect the asymptotic behaviour of their evolution in time. To have sufficient control on the smoothness of the data "at infinity", we shall strengthen our requirements. The conformal structure of the metric $\tilde{h}$ constitutes a basic part of the initial data. Apart from the condition of asymptotical flatness, it can be prescribed freely. Our requirements are concerned with the detailed behaviour of the conformal structure near infinity. We assume $(\tilde{S}, \tilde{h})$ to be "asymptotically Euclidean and regular" in the following sense.

There exists a three-dimensional, orientable, smooth, compact Riemannian manifold $(S, h)$ with points $i_{k} \in S, k=1,2, \ldots, q, q$ some positive integer, a diffeomorphism $\Phi$ of 
$S \backslash\left\{i_{1}, \ldots, i_{q}\right\}$ onto $\tilde{S}$, and a function $\Omega \in C^{2}(S) \cap C^{\infty}\left(S \backslash\left\{i_{1}, \ldots, i_{q}\right\}\right)$ with the following properties:

$$
\begin{aligned}
& \Omega=0, \mathrm{~d} \Omega=0, \operatorname{Hess}(\Omega)=-2 h \text { at the points } i_{k}, \\
& \Omega>0 \text { on } S \backslash\left\{i_{1} \ldots, i_{q}\right\} . \\
& h=\Omega^{2} \Phi_{*} \tilde{h} \quad \text { on } S \backslash\left\{i_{1}, \ldots, i_{q}\right\} .
\end{aligned}
$$

Suitable punctured neighbourhoods of the points $i_{k}$ correspond to the asymptotically flat ends of $(\tilde{S}, \tilde{h})$. Thus each point $i_{k}$ represents a space-like infinity. We shall be concerned with the precise behaviour of certain fields near a given space-like infinity. Therefore. we shall assume, without loss of generality, that there is only one asymptotically flat end. The point representing space-like infinity will be denoted by $i$. We shall consider in the following $\tilde{S}$ to be identified by $\Phi$ with $S \backslash\{i\}$. The set $S$ was assumed to be compact because of the special interest of this case.

Not all compact Riemannian spaces $(S, h)$ can give rise to Riemannian manifolds $(\tilde{S}=$ $S \backslash\{i\}, \tilde{h}=\Omega^{-2} h$ ) which are asymptotically Euclidean and regular and satisfy for suitable choices of $\Omega$ and $\tilde{\chi}$ the constraint equations [2]. To ensure the possibility, we require that $(S, h)$ has positive Yamabe number

$$
Y(h) \equiv-\inf _{\phi \in C^{\prime}(S), \phi>0} \frac{\int_{S}\left(8 h^{\alpha \beta} D_{\alpha} \phi D_{\beta} \phi+r_{h} \phi^{2}\right) \mathrm{d} \mu_{h}}{\left(\int_{S} \phi^{6} \mathrm{~d} \mu_{h}\right)^{1 / 3}} .
$$

With this assumption the scaling of the metric $h$, which determines only the conformal structure of $\tilde{h}$, may be chosen (cf. [18,22]) such that its Ricci scalar satisfies

$$
r_{h}<0 \text { on } S .
$$

Later we shall be led to consider further conditions on the conformal structure of $(S, h)$ in arbitrarily small neighbourhoods of the point $i$. That those conditions entail only rather mild restrictions in the class of three-dimensional conformal structures with positive Yamabe number will then be seen from the following lemma.

Lemma 2.1. Suppose $(N, k)$ is a smooth three-dimensional Riemannian manifold of signature $(-,-,-)$ and $p$ a point in $N$. Then, for $0<\epsilon \leq a$, with some small positive number $a$, we find smooth metrics $h_{\epsilon}$ and a smooth positive function $\omega$ on $S$ with the following properties. There exist open neighbourhoods $V_{\epsilon}, W_{\epsilon}$ of $i$, satisfying $V_{\epsilon} \subset W_{\epsilon}$ and $W_{\epsilon} \rightarrow\{i\}$ as $\epsilon \rightarrow 0$, and a diffeomorphism $\Psi$ of $V_{a}$ onto an open neighbourhood of $p$ such that:

(i) $h_{\epsilon}$ is conformal to $\Psi_{*} k$ on $V_{\epsilon}$,

(ii) $h_{\epsilon}=\omega^{2} h$ on $S \backslash W_{\epsilon}$.

(iii) in the $C^{2}$-topology on the set of smooth metrics on $S$ we have $h_{\epsilon} \rightarrow \omega^{2} h$ as $\epsilon \rightarrow 0$,

(iv) the metrics $h_{\epsilon}$ have Yamabe number $Y\left(h_{\epsilon}\right)>0$.

A metric can always be conformally rescaled in a neighbourhood of a given point such that the Ricci tensor of the rescaled metric vanishes at that point. In three dimensions 
the curvature tensor is determined by the Ricci tensor. Thus we can find smooth positive conformal factors $\omega$ and $\omega_{k}$ on $S$ and $N$, respectively, such that the curvature tensors of the metrics $h^{\prime}=\omega^{2} h, k^{\prime}=\omega_{k}^{2} k$ satisfy $r_{j k l m}\left[h^{\prime}\right]=0$ at $i, r_{j k l m}\left[k^{\prime}\right]=0$ at $p$.

Choose $a>0$ sufficiently small such that the $h^{\prime}$-metric ball $B_{3 a}(i)$ is an $h^{\prime}$-normal neighbourhood of $i$ and the $k^{\prime}$-metric ball $B_{3 a}(p)$ is a $k^{\prime}$-normal neighbourhood of $p$. Denote by $x^{\alpha}$ a system of $h^{\prime}$-normal coordinates on $B_{3 a}(i)$ centred at $i$ which is based on an $h^{\prime}$-orthonormal frame and let $x^{\alpha^{\prime}}$ be an analogous system of $k^{\prime}$-normal coordinates on $B_{3 a}(p)$. Expressing the metrics in these coordinates, we have $h_{\alpha \beta}^{\prime}=-\delta_{\alpha \beta}+\tilde{h}_{\alpha \beta}$, $k_{\alpha^{\prime} \beta^{\prime}}^{\prime}=-\delta_{\alpha^{\prime} \beta^{\prime}}+\tilde{k}_{\alpha^{\prime} \beta^{\prime}}$ with

$$
\tilde{h}_{\alpha \beta}=\mathrm{O}\left(|x|^{3}\right), \quad \tilde{k}_{\alpha^{\prime} \beta^{\prime}}=\mathrm{O}\left(\left|x^{\prime}\right|^{3}\right) \quad \text { as }|x|,\left|x^{\prime}\right| \rightarrow 0 .
$$

We denote by $\Psi$ the diffeomorphism of $B_{3 a}(i)$ onto $B_{3 a}(p)$ whose local expression in the normal coordinates is given by $\Psi^{\alpha^{\prime}}\left(x^{\beta}\right)=x^{\alpha^{\prime}}$. Choosing $f \in C^{\infty}(\mathbb{R}, \mathbb{R})$ with $f^{\prime} \leq 0$, $f(z)=1$ for $z \leq 1$, and $f(z)=0$ for $z \geq 2$, we define for given $\epsilon>0$ the function $\psi_{\epsilon} \in C_{0}^{\infty}\left(\mathbb{R}^{3}, \mathbb{R}\right)$ by $\psi_{\epsilon}(x)=f(|x| / \epsilon)$. A family of smooth metrics is then given by

$$
h_{\epsilon}=\psi_{\epsilon} \Psi_{*} k^{\prime}+\left(1-\psi_{\epsilon}\right) h^{\prime}, \quad 0<\epsilon<a,
$$

where the first tensor field on the right-hand side vanishes by definition outside the support of the funtion $\psi_{\epsilon}$. These metrics satisfy

$$
\text { (i') } h_{\epsilon}=\left(\Psi_{*} \omega_{k}\right)^{2} \Psi_{*} k \quad \text { on } B_{\epsilon}(i), \quad \text { (ii') } h_{\epsilon}=\omega_{h}^{2} h \quad \text { on } S \backslash B_{2 \epsilon}(i)
$$

and thus have properties (i), (ii) asserted in the lemma.

Since the Yamabe number is an invariant of the conformal structure and defines a continuous functional on the set of smooth metrics on $S$ endowed with the $C^{2}$-topology (cf. [1]), condition (iv) follows from (iii) once we have chosen the number $a$ small enough.

Because of property (ii'), it is sufficient to control the behaviour of the metrics $h_{\epsilon}$ to second order in terms of the coordinates $x^{\alpha}$ on $B_{3 a}(i)$. We identify $\omega_{k}^{2} k$ with $\Psi_{*}\left(\omega_{k}^{2} k\right)$ on $B_{3 a}(i)$. We observe that

$$
\left|\psi_{\epsilon}(x)\right| \leq C, \quad\left|\partial_{\alpha} \psi_{\epsilon}(x)\right| \leq \frac{C}{\epsilon}, \quad\left|\partial_{\alpha} \partial_{\beta} \psi_{\epsilon}(x)\right| \leq \frac{3 C}{\epsilon^{2}}
$$

for some constant $C$ and, by (2.9), we find on $B_{3 a}(i)$

$$
\left|\partial_{\mu_{1}}^{p_{1}} \partial_{\mu_{2}}^{p_{2}} \partial_{\mu_{3}}^{p_{3}}\left(h_{\alpha \beta}(x)-\left(\omega_{k}^{2} k\right)_{\alpha \beta}(x)\right)\right| \leq K \epsilon^{(3-p)}, \quad p \equiv p_{1}+p_{2}+p_{3}=0,1,2 .
$$

with some constant $K$. This implies our result.

Remarks. In a similar way we can obtain metrics with positive Yamabe number which have prescribed conformal structure near given points $i_{k} \in S, 1 \leq k \leq q$. The set of such metrics is dense in the set of smooth metrics with respect to the $C^{2}$-topology. If the metric $h$ is analytic in normal coordinates near $i$ and the metric $k$ is analytic in normal coordinates near $p$, the metrics $h_{\epsilon}$ can be arranged to be analytic near $i$. It should be noted that due to the constraint equations a change of the conformal structure of $\tilde{h}$ near space-like infinity entails a change of the metric $\tilde{h}$ in finite regions. 


\subsection{The conformal constraint equations}

Later we shall discuss the existence of solutions to Einstein's equation (2.1) by rewriting it as an equation for a suitably chosen conformal factor $\Omega$ and the conformal metric

$$
g_{\mu \nu}=\Omega^{2} \tilde{g}_{\mu \nu} .
$$

The first and second fundamental forms determined by the metrics $g_{\mu{ }^{\prime}}, \tilde{g}_{\mu \mu^{\prime}}$ on $\tilde{S}$ are then related by

$$
h_{\alpha \beta}=\Omega^{2} \tilde{h}_{\alpha \beta}, \quad \chi_{\alpha \beta}=\Omega\left(\tilde{\chi}_{\alpha \beta}+\Sigma \tilde{h}_{\alpha \beta}\right),
$$

where the function $\Sigma$ on $\tilde{S}$ denotes the derivative of $\Omega$ in the direction of the future directed $g$-unit normal of $\tilde{S}$. This entails for the traces $\chi=h^{\alpha \beta} \chi_{\alpha \beta}, \tilde{\chi}=\tilde{h}^{\alpha \beta} \tilde{\chi}_{\alpha \beta}$ the relation

$$
\Omega \chi=\tilde{\chi}+3 \Sigma .
$$

In terms of the conformal fields the constraint equations (2.2) take the form (cf. [10])

$$
\begin{aligned}
& 2 \Omega D_{\alpha} D^{\alpha} \Omega-3 D_{\alpha} \Omega D^{\alpha} \Omega+\frac{1}{2} \Omega^{2} r-3 \Sigma^{2} \\
& \quad-\frac{1}{2} \Omega^{2}\left\{\left(\chi_{\alpha}{ }^{\alpha}\right)^{2}-\chi_{\alpha \beta} \chi^{\alpha \beta}\right\}+2 \Omega \Sigma \chi_{\alpha}{ }^{\alpha}=0, \\
& \Omega^{3} D^{\alpha}\left(\Omega^{-2} \chi_{\alpha \beta}\right)-\Omega\left\{D_{\beta}\left(\chi_{\alpha}{ }^{\alpha}\right)-2 \Omega^{-1} D_{\beta} \Sigma\right\}=0 .
\end{aligned}
$$

where $D$ denotes the Levi-Civita connection and $r$ the Ricci scalar of the metric $h$.

We shall assume in the following that

$$
\Sigma=0 . \quad \chi_{\alpha}{ }^{\alpha}=0 \quad \text { on } \tilde{S} .
$$

The first condition is concerned with the choice of the conformal factor which is completely at our disposal so far. Both conditions together say that the hypersurface $\tilde{S}$ is maximal in $\tilde{M}$ with respect to the metrics $\tilde{g}_{\mu \nu}, g_{\mu \nu}$. With these assumptions the conformal constraints on $\tilde{S}$ reduce to

$$
\begin{aligned}
& \left(D_{\alpha} D^{\alpha}-\frac{1}{8} r\right) \theta=\frac{1}{8} \chi_{\alpha \beta} \chi^{\alpha \beta} \theta \quad \text { with } \theta=\Omega^{-1 / 2}, \\
& D^{\alpha}\left(\Omega^{-2} \chi_{\alpha \beta}\right)=0 .
\end{aligned}
$$

These equations suggest constructing initial data $h_{\alpha \beta}, \chi_{\alpha \beta}, \Omega$ directly, without referring first to the fields $\tilde{h}_{\alpha \beta}, \tilde{\chi}_{\alpha \beta}$. The known technique (cf. [5]) to construct solutions to the vacuum constraints (2.2) suggests the following procedure:

(i) Choose a smooth negative definite metric $h$ on a three-dimensional, orientable, smooth. compact manifold $S$, pick a point $i \in S$ and set $\tilde{S}=S \backslash\{i\}$.

(ii) Find a smooth, symmetric, tensor field $\psi_{\alpha \beta}$ on $\tilde{S}$ which is trace-free with respect to $h$ and satisfies

$$
D^{\alpha} \psi_{\alpha \beta}=0
$$


Such tensor fields may be obtained by performing a York-splitting [26] as follows: Choose a smooth, symmetric, trace-free tensor field $\psi_{\alpha \beta}^{\prime}$ on $\tilde{S}$ and set

$$
\psi_{\alpha \beta}=D_{\alpha} X_{\beta}+D_{\beta} X_{\alpha}-\frac{2}{3} h_{\alpha \beta} D_{\gamma} X^{\gamma}+\psi_{\alpha \beta}^{\prime},
$$

where $X_{\alpha}$ is some 1-form on $\tilde{S}$. Eq. (2.15) then implies an elliptic system of PDEs for $X_{\alpha}$ which under suitable assumptions can be solved to obtain $\psi_{\alpha \beta}$.

(iii) Set $\chi_{\alpha \beta}=\theta^{-4} \psi \psi_{\alpha \beta}$ in Eq. (2.13) and find on $\tilde{S}$ a positive solution $\theta$ of the resulting Lichnerowicz equation

$$
\left(D_{\alpha} D^{\alpha}-\frac{1}{8} r\right) \theta=\frac{1}{8} \psi_{\alpha \beta} \psi^{\alpha \beta} \theta^{-7} .
$$

The fields $h, \Omega=\theta^{-2}$, and $\chi_{\alpha \beta}=\Omega^{2} \psi_{\alpha \beta}$ then provide a solution to the conformal constraints on $\tilde{S}$.

In this procedure the freedom to prescribe the function $\Omega$ is reflected in the freedom to determine the scaling of the metric $h$. If $\phi$ is a positive function on $S$ the transition

$$
h_{\alpha \beta} \rightarrow \phi^{4} h_{\alpha \beta}, \quad \psi_{\alpha \beta} \rightarrow \phi^{-2} \psi_{\alpha \beta}, \quad \Omega \rightarrow \phi^{2} \Omega, \quad \chi_{\alpha \beta} \rightarrow \phi^{2} \chi_{\alpha \beta}
$$

yields another solution to Eqs. (2.13)-(2.16) which corresponds to the same physical data.

Since we considered Eqs. (2.15) and (2.16) only on $\tilde{S}$, we need to specify the behaviour of the solutions (respectively of $\psi_{\alpha \beta}^{\prime}$ and $X_{\alpha}$ ) near the point $i$. Assume $a>0$ small enough such that the $h$-metric ball $B_{2 a}(i)$ is a strictly convex normal neighbourhood of $i$ and let $x^{\alpha}$ be normal coordinates with origin at $i$ which are based on an $h$-orthonormal frame $\mathrm{e}_{k}$ at $i$. Condition (2.6) entails that $\Omega=|x|^{2} f(x)$ near $i$, where $f$ is a continuous function with $f(0)=1$. Thus we need to impose the condition

$$
|x| \theta \rightarrow 1 \quad \text { as } x \rightarrow 0 .
$$

If we define on $B_{a}(i) \backslash\{i\}$ coordinates $y^{\alpha}=x^{\alpha} /|x|^{2}$ and write $\tilde{h}=\Omega^{-2} h=\tilde{h}_{\alpha \beta} \mathrm{d} y^{\alpha} \mathrm{d} y^{\beta}$, we find $\tilde{h}_{\alpha \beta}=-\delta_{\alpha \beta}+O(1 /|y|)$ as $|y| \rightarrow \infty$. Thus $\tilde{h}$ satisfies a basic requirement of asymptotic euclidicity. Later we shall be more specific about the coefficient of $1 /|y|$ in the expansion of $\tilde{h}_{\alpha \beta}$ in terms of $1 /|y|^{p}$. It determines the ADM-energy

$$
m=E=\frac{1}{16 \pi} \lim _{r \rightarrow \infty} \int_{S_{r}} \delta^{\alpha \beta}\left(\partial_{\alpha} \tilde{h}_{\beta \gamma}-\partial_{\gamma} \tilde{h}_{\alpha \beta}\right) n^{\gamma} \mathrm{d} S_{r} .
$$

Here $S_{r}$ denotes the 2-sphere $\{|y|=r\}, \mathrm{d} S_{r}$ the $\tilde{h}$-area element on it, and $n$ the outward directed $\tilde{h}$-unit normal to $S_{r}$.

The physical second fundamental form $\tilde{\chi}_{\alpha \beta}$, the conformal fundamental form $\chi_{\alpha \beta}$, and the solution $\psi_{\alpha \beta}$ to (2.15) are related by

$$
\tilde{\chi}_{\alpha \beta}=\Omega^{-1} \chi_{\alpha \beta}=\Omega \psi_{\alpha \beta} .
$$

Let $\tilde{\chi}_{\alpha \beta}$ be given in the coordinates $y^{\alpha}$. Then (2.4) suggests that $\tilde{\chi}_{\alpha \beta}=\mathrm{O}\left(1 /|y|^{2}\right)$ as $|y| \rightarrow \infty$ on $B_{a}(i) \backslash\{i\}$. This entails for the coefficients $\chi_{\alpha \beta}, \psi_{\alpha \beta}$ in the coordinates $x^{\alpha}$ the conditions 


$$
\chi_{\alpha \beta}=\mathrm{O}(1), \quad \psi_{\alpha \beta}=\mathrm{O}\left(\frac{1}{|x|^{4}}\right) \quad \text { as } x \rightarrow 0 .
$$

In the case where the solution $\psi_{\alpha \beta}$ to Eq. (2.15) extends smoothly to all of $S$ the coefficients $\tilde{\chi}_{\alpha \beta}$, given in the coordinates $y^{\alpha}$, satisfy the strong fall-off conditions $\tilde{\chi}_{\alpha \beta}=\mathrm{O}\left(1 /|y|^{6}\right)$ as $|y| \rightarrow \infty$. If we wish to consider data for which the ADM-momentum and the ADM-angular momentum, given by

$$
\begin{aligned}
& P_{\alpha}=\frac{1}{8 \pi} \lim _{r \rightarrow \infty} \int_{S_{r}}\left(\tilde{\chi}_{\alpha \beta}-\tilde{\chi} \tilde{h}_{\alpha \beta}\right) n^{\beta} \mathrm{d} S_{r}, \\
& J_{\alpha}=\frac{1}{8 \pi} \lim _{r \rightarrow \infty} \int_{S_{r}} \tilde{\epsilon}_{\alpha \beta \gamma} y^{\beta}\left(\tilde{\chi}^{\gamma \rho}-\tilde{\chi} \tilde{h}^{\gamma \rho}\right) n_{\rho} \mathrm{d} S_{r},
\end{aligned}
$$

respectively, do not vanish, we would need to analyse fields $\psi_{\alpha \beta}$ which are singular at $i$.

Though a detailed discussion of the smoothness of the second fundamental form $\chi_{\alpha \beta}$ near $\{i\}$ may be a delicate matter, the source of our main problem lies elsewhere. If we try to analyse the time evolution of the data in terms of the "regular conformal field equations" deduced in [7,8], we have to consider as unknowns a number of tensor fields derived from the basic data $h_{\alpha \beta}, \chi_{\alpha \beta}$, and $\Omega$. The data on $S$ for the conformal field equations in the form used in this article are listed in (2.27), (2.31)-(2.33), (2.35), (2.36), (2.38) and (2.39). To determine the electric and magnetic part (2.38) and (2.39) of the rescaled Weyl tensor. which represent in a sense the most important data, we have to divide by powers of $\Omega$. This leads to singularities in the fields at $i$ which cannot be avoided unless the ADM-energy $m$ of the data vanishes, i.e. unless the conformal Weyl tensor vanishes everywhere on $\tilde{S}$ and we deal with Minkowski space (cf. $[22,25]$ ). These "structural singularities" pose the essential problem in any detailed analysis of the time evolution of the fields near space-like infinity. The best we can do to simplify the analysis is to impose smoothness conditions near $i$ to avoid the occurrence of "spurious singularities" in the evolution, which may arise from unsatisfactory regularity properties of the metric and the second fundamental form at space-like infinity.

In this article we shall use the conformal representation of Einstein's equation introduced in [12] to analyse the time evolution near space-like infinity. Though the focus of our investigation will be on the structure of the evolution equations contained in the conformal field equations, we shall need quite detailed information on the behaviour of the data near $i$. To simplify the discussion we shall therefore consider in this article only the time-symmetric case

$$
\chi_{\alpha \beta}=0, \quad \tilde{\chi}_{\alpha \beta}=0 \quad \text { on } \tilde{S} .
$$

The comparison of expressions (2.38) and (2.39) with expression (2.40), which is obtained under the assumption above, shows that this leads to considerable simplifications.

Nevertheless, the essential difficulty which arises from an ADM-energy $m>0$ will still be present as is seen from expressions (2.42) and (2.43) which take into account (2.19). We shall see that even with (2.19) and the smoothness assumption on the metric $h$, there will 
be, depending on the choice of $h$, interesting distinctions in the asymptotic behaviour of the time evolution of the data.

The construction of constrained inital data is now reduced to solving (2.16) on $\tilde{S}$ with vanishing right-hand side such that condition (2.17) is satisfied. For convenience we shall assume that

\section{$h$ is analytic near $i$}

in the sense that the metric coefficients of $h$ with respect to the normal coordinates $x^{\alpha}$ on $B_{2 a}(i)$ are real analytic functions if $a$ is chosen small enough.

Under conformal rescalings $h \rightarrow h^{\prime}=\phi^{2} h$ with smooth positive conformal factors the Yamabe operator $L_{h}[u]=\left(h^{\alpha \beta} D_{\alpha} D_{\beta}-\frac{1}{8} r_{h}\right)[u]$ transforms as

$$
\phi^{-(n+2) / 2} L_{h}[u]=L_{h^{\prime}}\left[\phi^{-(n-2) / 2} u\right] .
$$

This allows us to scale $h$ such that (2.8) holds. We use Hadamard's construction to obtain a parametrix for $L \equiv L_{h}$. The function $\Gamma=|x|^{2}$ is analytic on $B_{2 a}(i)$ and satisfies

$$
D_{\alpha} \Gamma D^{\alpha} \Gamma=-4 \Gamma
$$

A function $U\left(x^{\alpha}\right)$ on $B_{2 a}(i)$ which is analytic and satisfies $U(i)=1$ and $L\left[U \Gamma^{-1 / 2}\right]=0$ on $B_{2 a}(i) \backslash\{i\}$ is obtained as follows (cf. [14]). The equation

$$
0=-\Gamma^{3 / 2} L\left[U \Gamma^{-1 / 2}\right]=D^{\alpha} \Gamma D_{\alpha} U+\frac{1}{2}\left(D^{\alpha} D_{\alpha} \Gamma+6\right) U-\Gamma L[U]
$$

will be satisfied on $B_{2 a}(i) \backslash\{i\}$ by a function of the form

$$
U=\sum_{p=0}^{\infty} U_{p} \Gamma^{p},
$$

if the coefficient functions $U_{p}$ are analytic, if they solve the equations

$$
\begin{aligned}
D^{\alpha} \Gamma D_{\alpha} U_{0}= & -\frac{1}{2}\left(D^{\alpha} D_{\alpha} \Gamma+6\right) U_{0}, \quad U(i)=1, \\
D^{\alpha} \Gamma D_{\alpha} U_{p}= & -\frac{1}{2}\left(D^{\alpha} D_{\alpha} \Gamma+6-4 p\right) U_{p} \\
& -\frac{1}{2 p-1} L\left[U_{p-1}\right], \quad p=1,2, \ldots
\end{aligned}
$$

and if they define a convergent series on $B_{2 a}(i)$ by (2.24). Such functions are determincd recursively by

$$
\begin{aligned}
& U_{0}=\exp \left\{\frac{1}{4} \int_{0}^{\Gamma^{1 / 2}}\left(D_{\alpha} D^{\alpha} \Gamma+6\right) \frac{\mathrm{d} s}{s}\right\} \\
& U_{p \mid 1}=-\frac{U_{0}}{(4 p-2) \Gamma^{(p+1) / 2}} \int_{0}^{\Gamma^{1 / 2}} \frac{L\left[U_{p}\right] s^{p}}{U_{0}} \mathrm{~d} s, \quad p=0,1, \ldots
\end{aligned}
$$


where the integration is performed in terms of the affine parameter $s=\Gamma^{1 / 2}=|x|$ along the geodesics emanating from $i$. Notice that the function $U$ is determined completely in terms of the local geometry near $i$

We now choose a function $\theta^{*} \in C^{\infty}(\tilde{S})$ which coincides with $U \Gamma^{-1 / 2}$ on $B_{a}(i) \backslash\{i\}$. Then $L\left[\theta^{*}\right]=f$ on $\tilde{S}$ with some function $f \in C^{\infty}(S)$ which vanishes on $B_{a}(i)$. To obtain the solution $\theta$ to our problem we need to add a function which contains information on the global geometry. We find a weak solution $W$ to the equation $L[W]=-f$ on $S$ by observing that in the weak form

$$
\int_{S}\left(-D^{\alpha} W D_{\alpha} \phi-\frac{1}{8} r W \phi\right) \mathrm{d} \mu_{h}=-\int_{S} f \phi \mathrm{d} \mu_{h} . \quad \phi \in C^{\infty}(S),
$$

of this equation the left-hand side defines a bilinear form which is continuous and coercive on the Sobolev space $H^{1,2}(S)$. The existence of a unique weak solution $W \in H^{1,2}(S)$ to our equation thus follows by the Lax-Milgram theorem (cf. [15]). Elliptic regularity theory shows that this solution is smooth on $S$ and analytic on $B_{a}(i)$ where it satisfies $L[W]=0$. The function $\theta=\theta^{*}+W$ is the unique solution to (2.16) and (2.17). Since $\theta>0$ on a punctured neighbourhood of $i$ it follows from (2.8) and the strong maximum principle that $\theta>0$ on $\tilde{S}$. Using again the inversion as coordinate transformation we find that $\tilde{h}$ is of the form (2.3) with ADM-energy $m=2 W(i)$.

The function $\theta^{\prime}=\phi^{-1 / 2} \theta$ solves the Yamabe equation with respect to the metric $h^{\prime}=$ $\phi^{2} h$ and $\tilde{h}=\Omega^{-2} h=\Omega^{\prime}{ }^{-2} h^{\prime}$ is independent of the scaling condition (2.8).

Near $i$ let $x^{\alpha^{\prime}}$ be the $h^{\prime}$-normal coordinates with origin at $i$ based on the $h^{\prime}$-orthonormal frame $e_{k}^{\prime}=\phi^{-1} \mathrm{e}_{k}$. The relation $\delta^{\alpha}{ }_{k} \partial_{x^{\alpha}}=\mathrm{e}_{k}=\phi e_{k}^{\prime}=\phi \delta^{\alpha^{\prime}}{ }_{k}\left(\partial x^{\alpha} / \partial x^{\alpha^{\prime}}\right) \partial_{x^{\alpha}}$ at $i$ entails $x^{\alpha}\left(x^{\alpha^{\prime}}\right)=x^{\alpha^{\prime}}\left(\phi(i)^{-1}+\mathrm{O}\left(\left|x^{\prime}\right|\right)\right)$. This implies that $\theta^{\prime}=\phi^{-1 / 2} \theta=\phi^{-1 / 2}(U /|x|+W)$ has the form $\theta^{\prime}=U^{\prime} /\left|x^{\prime}\right|+W^{\prime}$ near $i$ with $U^{\prime}(i)=1$ if and only if $\phi(i)=1$ and in this case we have $2 W^{\prime}(i)=2 W(i)$. In other words, if we fix our procedure of solving the constraints by imposing condition (2.17), we are only free to choose $h$ in the subclass of metrics in the conformal structure of $h$ which all coincide at $i$. Transition to another such class results in a rescaling of the "physical" metric $\tilde{h}$ by a constant conformal factor.

\subsection{Initial data for the conformal Einstein equations}

To study the propagation of the fields, we shall use the conformal representation of Einstein's equation (2.1) in the form discussed in [12]. Here the equation is considered as an equation for the metric $g$ in the conformal class of the "physical" metric $\tilde{g}$, where the conformal factor in (2.10) is fixed by some gauge condition, and a connection $\hat{\nabla}$ which preserves the conformal structure in the sense that parallel transport maps conformal frames onto conformal frames. The connection $\hat{\nabla}$ need not be metric for any metric in the conformal class of $\tilde{g}$. The following associated fields appear as unknowns in the equations:

(i) The coefficients

$$
\mathrm{e}^{\mu}{ }_{k}=\left\langle\mathrm{d} x^{\mu}, \mathrm{e}_{k}\right\rangle, \quad k, \mu=0,1,2,3 .
$$


of a local frame $\mathrm{e}_{k}$ with respect to some local space-time coordinates $x^{\mu}$. We require that $g\left(\mathrm{e}_{j}, \mathrm{e}_{k}\right)=\eta_{j k}$. The coordinates and the frame are subject to gauge conditions which will be discussed in detail later on. It will be assumed that

$$
x^{0}=0 \quad \text { on } \tilde{S}, \quad \mathrm{e}_{0} \text { is future directed and normal to } \tilde{S},
$$

such that $\mathrm{e}_{k}^{0}=0$ for $k=1,2,3$. In the following all spatial tensors on $S$ will be expressed with respect to the local frame $\mathrm{e}_{j}, j=1,2,3$, on $S$.

(ii) The connection coefficients $\hat{\Gamma}_{i}{ }^{j}{ }_{k}$ of $\hat{\nabla}$ in the frame $\mathrm{e}_{k}$ which satisfy $\hat{\nabla}_{\mathrm{e}_{i}} \mathrm{e}_{k}=\hat{\Gamma}_{i}{ }_{k} c_{j}$. Since the connection respects the conformal structure we can write

$$
\hat{\Gamma}_{i}^{j}{ }_{k}=\Gamma_{i}{ }_{k}{ }^{j}+\delta^{j}{ }_{i} f_{k}+\delta^{j}{ }_{k} f_{i}-\eta_{i k} \eta^{j l} f_{l} \text {, }
$$

where the $\Gamma_{i}{ }_{k}$ are the connection coefficients in the frame $\mathrm{e}_{k}$ of the Levi-Civita connection $\nabla$ of $g$ and

$$
f_{k}=\frac{1}{4} \hat{\Gamma}_{k}{ }^{j}{ }_{j}
$$

The 1-form $f_{k}$, which is exact if and only if $\hat{\nabla}$ is locally the Levi-Civita connection of a metric in the conformal class, is subject to gauge conditions. We shall require that

$$
\hat{\Gamma}_{0}{ }^{j} k=0, \quad j, k=0,1,2,3 \text { on } S
$$

and assign certain values to the functions $f_{j}, j=1,2,3$ on $\tilde{S}$. Then we have on $S$

$$
\begin{aligned}
& \hat{\Gamma}_{k}{ }^{0}{ }_{0}=0, \quad \hat{\Gamma}_{i}{ }^{j}{ }_{k}=\Gamma_{i}{ }_{k}{ }_{k}+\delta^{j}{ }_{i} f_{k}+\delta^{j}{ }_{k} f_{i}-h_{i k} h^{j \prime} f_{l} \\
& -\hat{\Gamma}_{i}{ }^{0}{ }_{k} h^{k j}=\hat{\Gamma}_{i}{ }^{j}{ }_{0}=\chi_{i}{ }^{j}=\chi_{\alpha} \beta \mathrm{e}^{\alpha}{ }_{i} \mathrm{e}^{\beta}{ }_{k} h^{k j}, \quad i, j, k=1,2,3 .
\end{aligned}
$$

with

$$
\Gamma_{i}^{j}{ }_{k} \mathrm{e}_{j}=D_{\mathrm{e}_{i}} \mathrm{e}_{k}, \quad \chi_{i j}=g\left(\hat{\nabla}_{\mathrm{e}_{i}} \mathrm{e}_{0}, \mathrm{e}_{j}\right)
$$

With assumption (2.19) the data for the connection coefficients are given completely by the connection coefficients for the interior connection $D$ and the functions $f_{j}$ on $\tilde{S}$.

(iii) The tensor

$$
A_{j k}=\frac{1}{2} \hat{R}_{(j k)}-\frac{1}{12} \eta^{i l} \hat{R}_{i l} \eta_{j k}+\frac{1}{4} \hat{R}_{[j k]}
$$

derived from the Ricci tensor $\hat{R}_{j k}$ of $\hat{\nabla}$. It will be subject to the gauge condition

$$
A_{j 0}=0 .
$$

The remaining coefficients will be given on $\tilde{S}$ in our choice of gauge by

$$
\begin{aligned}
& A_{j k}=-\Omega^{-1}\left\{D_{j} D_{k} \Omega-\frac{1}{3} D_{l} D^{l} \Omega h_{j k}\right\}+\frac{1}{12}\left\{r-\left(\chi_{l}^{l}\right)^{2}+\chi_{i l} \chi^{i l}\right\} h_{j k}, \\
& A_{0 k}=\Omega^{-1} \chi_{k}^{l} D_{l} \Omega, \quad i, j, k, l=1,2,3,
\end{aligned}
$$

such that $A_{j k}$ is represented under assumption (2.19) by the spatial tensor

$$
A_{j k}=-\Omega^{-1}\left\{D_{j} D_{k} \Omega-\frac{1}{3} D_{l} D^{l} \Omega h_{j k}\right\}+\frac{1}{12} r h_{j k} .
$$

All other projections vanish on $\tilde{S}$. 
(iv) The "rescaled Weyl tensor" $d^{i}{ }_{j k l}=\Omega^{-1} C^{i}{ }_{j k l}$, where $C^{i}{ }_{j k l}$ is the Weyl tensor of $g$. It is specified on $\tilde{S}$ in terms of its electric part

$$
\begin{aligned}
d_{i j} \equiv d_{i 0 j 0}= & \Omega^{-2}\left\{D_{i} D_{j} \Omega-\frac{1}{3} h_{i j} D^{k} D_{k} \Omega+\Omega s_{i j}\right\} \\
& -\Omega{ }^{1}\left\{\chi^{k}{ }_{k} \chi_{i j}-\chi^{k}{ }_{i} \chi_{k j}-\frac{1}{3} h_{i j}\left(\left(\chi^{k}{ }_{k}\right)^{2}-\chi^{k l} \chi_{k l}\right)\right\}
\end{aligned}
$$

and its magnetic part

$$
d_{i j}^{*} \equiv d_{i 0 j 0}^{*}=-\Omega^{-1} D_{k} \chi_{l(i} \epsilon_{j)}^{k l},
$$

which are both spatial tensors on $\tilde{S}$. Here $s_{j k}$ denotes the trace-free part of the Ricci tensor of $h$ and $\epsilon_{i j k}$ the corresponding Levi-Civita symbol. Under assumption (2.19) these data reduce to

$$
d_{i j} \equiv d_{i 0 j 0}=\Omega^{-2}\left\{D_{i} D_{j} \Omega-\frac{1}{3} h_{i j} D^{k} D_{k} \Omega+\Omega s_{i j}\right\}, \quad d_{i j}^{*}=0 .
$$

Using the local form $\Omega=\Gamma /(U+|x| W)^{2}$, we get near $i$ a more detailed expression for the field above. If $d_{i j}$ is represented in the space-spinor notation by the spinor field $\phi_{a b c d}$, we get a splitting

$$
\phi_{a b c d}=\phi_{a b c d}^{\prime}+\phi_{a b c d}^{W}
$$

Here

$$
\begin{aligned}
\phi_{a b c d}^{\prime}= & \Omega^{\prime}-2\left\{D_{(a b} D_{c d)} \Omega^{\prime}+\Omega^{\prime} s_{a b c d}\right\} \\
= & \Gamma^{-2}\left\{U^{2} D_{(a b} D_{c d)} \Gamma-4 U D_{(a b} \Gamma D_{c d)} U\right. \\
& \left.-2 \Gamma U D_{(a b} D_{c d)} U+6 \Gamma D_{(a b} U D_{c d)} U+\Gamma U^{2} s_{a b c d}\right\}
\end{aligned}
$$

is the part derived from the function $\Omega^{\prime}=\Gamma / U^{2}$ which contains only information on the local geometry near $i$. The information on the global geometry is contained in

$$
\begin{aligned}
\phi_{a b c d}^{W}= & \Gamma^{-2}\left\{-\frac{3}{2} \frac{1}{|x|} U W D_{(a b} \Gamma D_{c d)} \Gamma+U W|x| D_{(a b} D_{c d)} \Gamma\right. \\
+ & 2|x|\left(W D_{(a b} \Gamma D_{c d)} U-3 U D_{(a b} \Gamma D_{c d)} W\right) \\
& +2|x| \Gamma\left(-U D_{(a b} D_{c d)} W-W D_{(a b} D_{c d)} U\right. \\
& \left.+6 D_{(a b} U D_{c d)} W+U W s_{a b c d}\right) \\
& \left.+\Gamma^{2}\left(-2 W D_{(a b} D_{c d)} W+6 D_{(a b} W D_{c d)} W+W^{2} s_{a b c d}\right)\right\} .
\end{aligned}
$$

We shall call $\phi_{a b c d}^{\prime}$ the "massless" and $\phi_{a b c d}^{W}$ the "massive" part of the rescaled Weyl tensor.

While under suitable assumptions on the metric $h$ the massless part can be extended to an analytic tensor field on $B_{a}(i)$, the massive part always hehaves like $O\left(\Gamma^{-3 / 2}\right)$ as $\Gamma \rightarrow 0$ unless $m=0$. If we admit cases where compact subsets of $\tilde{S}$ have been removed from our initial hypersurface, we may consider initial data with $\phi_{a b c d}^{W}=0$ but $\phi_{a b c d}^{\prime} \neq 0$ near $i$. 


\section{Normal expansions at space-like infinity}

We shall now consider a certain manifold $C_{a}$ and a certain type of expansion which will allow us a convenient discussion of data which are singular at $i$. The initial value problem near space-like infinity, to be discussed later, will also make use of the manifold $C_{a}$.

\subsection{The construction of $C_{a}$}

The construction of $C_{a}$ involves a blow up of the point $i$ to a sphere and an extension through this sphere. The following discussion will be given in analogy to a similar discussion in [9] to which we refer for further details. We shall employ the space-spinor formalism on $S$ in the notation used in [12]. It will be obvious from our subsequent considerations that for our purpose it is irrelevant which spin structure were chosen if there existed more than one. Let $S U(S)$ denote the bundle of normalized spin frames over $S$ with structure group $S U(2, C)$ and projection $\pi$ onto $S$. Later we shall assume that $\tilde{S}$ is a space-like hypersurface of a four-dimensional space-time and that the restriction of $S U(S)$ to $\tilde{S}$ arises as the set of spin frames $\delta=\left\{\delta_{a}\right\}_{a=0,1}$ which are normalized with respect to the alternating form $\epsilon$. such that

$$
\epsilon\left(\delta_{a}, \delta_{b}\right)=\epsilon_{a b}, \quad \epsilon_{01}=1
$$

as well as with respect to the Hermitian form on spinors which is defined by contraction with the future directed normal vector field $\tau$ of $\tilde{S}$, such that

$$
g\left(\tau, \delta_{a} \bar{\delta}_{a^{\prime}}\right)=\epsilon_{a}^{0} \epsilon_{a^{\prime}} 0^{\prime}+\epsilon_{a}^{1} \epsilon_{a^{\prime}} 1^{\prime} .
$$

Here we assume that $g(\tau, \tau)=2$ and $g$ denotes the space-time metric. Given with respect to such a spin frame, the vector $\tau$ has representation

$$
\tau^{a a^{\prime}}=\epsilon_{0}{ }^{a} \epsilon_{0^{\prime}}^{a^{\prime}}+\epsilon_{1}^{a} \epsilon_{1^{\prime}}^{a^{\prime}}
$$

which we will assume in the following. We use the realizations

$$
\begin{aligned}
& S L(2, C)=\left\{t^{a}{ }_{b} \in G L(2, C) \mid \epsilon_{a c} t^{a}{ }_{b} t^{c}{ }_{d}=\epsilon_{b d}\right\}, \\
& S U(2, C)=\left\{t^{a}{ }_{b} \in S L(2, C) \mid \tau_{a a^{\prime}} t^{a}{ }_{b} \bar{t}^{a^{\prime}}{ }_{b^{\prime}}=\tau_{b b^{\prime}}\right\} .
\end{aligned}
$$

If $\delta \in S U(S)$, the action of $t \in S U(2, C)$ maps $\delta$ onto $\delta t$ given by basis spinors $\delta_{b} t^{b}{ }_{a}$, $a=0,1$. Using the Van der Waerden symbols for space spinors

$$
\sigma_{j}^{a b}=\sigma_{j}^{(a}{ }_{a^{\prime}} \tau^{b) a^{\prime}}, \quad \sigma_{a b}^{j}=\tau_{(b} a^{a^{\prime}}{ }_{a) a^{\prime}}, \quad j=1,2,3,
$$

such that

$$
\begin{aligned}
& \delta_{k}^{j}=\sigma_{a b}^{j}{ }_{a k}^{a b}, \quad j=1,2,3, \quad \epsilon_{a}^{b} \epsilon_{a^{\prime}}^{b^{\prime}}=\frac{1}{2} \tau_{a a^{\prime}} \tau^{b b^{\prime}}+\sigma^{j}{ }_{a f} \tau_{a^{\prime}}^{f} \tau^{e b^{\prime}} \sigma_{j e}{ }^{b}, \\
& \eta_{j k} \sigma_{a b}^{j}{ }_{a b}^{k}{ }_{c d}=-\delta_{j k} \sigma_{a b}^{j} \sigma_{c d}^{k}=-\epsilon_{a(c} \epsilon_{d) b} \equiv h_{a b c d},
\end{aligned}
$$


the covering map onto the connected component of the rotation group is given by

$$
S U(2, C) \ni t^{a}{ }_{b} \stackrel{\Phi^{\prime \prime}}{\rightarrow} t^{i}{ }_{j}=\sigma^{i}{ }_{a b} t^{a}{ }_{c} t^{b}{ }_{d} \sigma_{j}{ }^{c d} \in \mathrm{O}_{+}(3, \mathbb{R}),
$$

the induced isomorphism of Lie algebras by

$$
s u(2, C) \ni u_{b}^{a} \stackrel{\Phi_{*}^{\prime \prime}}{\rightarrow} u_{j}^{i}=2 \sigma_{a b}^{i} u_{c}^{a} \sigma_{j}{ }^{c b} \in \mathrm{o}(3 . \mathbb{R}) .
$$

and the inverse of the latter by

$$
\mathrm{o}(3, \mathbb{R}) \ni u_{j}^{i} \stackrel{\Phi_{*}^{\prime \prime}-1}{\rightarrow} u^{a}{ }_{b}=\frac{1}{2} u^{i}{ }_{j} \sigma_{i}^{a c} \sigma^{j}{ }_{b c} \in \operatorname{su}(2, C) .
$$

Finally, the covering morphism of $S U(S)$ onto the bundle $\mathrm{O}_{+}(S)$ of positively oriented orthonormal frames on $S$ maps the frame $\delta \in S U(S)$ onto the frame on $S$ with vectors $\sigma_{j}{ }^{a b} \delta_{a} \delta_{b}, j=1,2,3$. We use this map to pull back to $S U(S)$ the Levi-Civita connection form on $S O(S)$. Combining this with the map $\Phi_{*}^{\prime \prime}-1$, the connection is represented by an $s u(2)$-valued connection form $\check{\omega}^{a} b$ on $S U(S)$. Similarly, pulling back the $\mathbb{R}^{3}$-valued solder form on $O_{+}(S)$ and contracting with the Van der Waerden symbols results in a 1-form $\sigma^{a b}$ on $S U(S)$ which we call the solder form on $S U(S)$.

Let $\check{H}$ denote the real horizontal vector field on $S U(S)$ satisfying $\left\langle\sigma^{a b} . \check{H}\right\rangle=\epsilon_{0}{ }^{(a} \epsilon_{1}{ }^{h)}$ or, equivalently.

$$
T_{\delta}(\pi) \check{H}(\delta)=\delta_{(0} \tau_{1)}{ }^{b^{\prime}} \bar{\delta}_{b^{\prime}}=\frac{1}{2}\left(\delta_{0} \bar{\delta}_{0^{\prime}}-\delta_{1} \bar{\delta}_{1^{\prime}}\right), \quad \delta \in S U(S) .
$$

It follows that $T_{\delta t}(\pi) \check{H}(\delta t)=T_{\delta}(\pi) \check{H}(\delta)$ if and only if

$$
t \in U(1) \equiv\left\{t \in S U(2, C) \mid t=\left(\begin{array}{cc}
\mathrm{e}^{\mathrm{i} \phi} & 0 \\
0 & \mathrm{e}^{-\mathrm{i} \phi}
\end{array}\right), \phi \in \mathbb{R}\right\}
$$

The construction of the manifold $C_{a}$ will be described now. Choose a fixed spin frame $\delta^{*}$ in the fibre $\pi^{-1}(i)$ over $i$. The action of the group on this spin frame defines a smooth parametrization $\delta(t)$ of the fibre in terms of $t \in S U(2, C)$ such that $\delta(e)=\delta^{*}$ with $e$ the unit in $S U(2, C)$. Choose $a>0$ such that the metric ball $B_{a}(i)$ in $S$ with centre $i$ and radius $a$ is strictly geodesically convex and that the metric $h$ is analytic on $B_{a}(i)$. Let $1-a, a[\exists \rho \rightarrow \delta(\rho, t) \in S U(S)$ be the integral curve of the vector field $\sqrt{2} \breve{H}$ satisfying $\delta(0, t)=\delta(t)$. We set $C_{a}=\{\delta(\rho, t) \in S U(S) \| \rho \mid<a, t \in S U(2, C)\}$. This set defines a smooth submanifold of $S U(S)$ diffeomorphic to ] $-a, a[\times S U(2, C)$ which is in fact an analytic submanifold of the restriction of $S U(S)$ to $B_{a}(i)$. Since the integral curves of $\sqrt{2} \breve{H}$ through $\pi^{-1}(i)$ project onto the geodesics through $i$ with tangent vector of unit length, the restriction of $\pi$ to $C_{a}$. denoted in the following by $\pi^{\prime}$, maps $C_{u}$ onto $B_{a}(i)$.

The action of $U(1)$ on $S U(S)$ implies an action of this group on $C_{a}$. While $I^{0} \equiv \pi^{-1}(i)=$ $\{\rho=0\}$ is diffeomorphic to $S U(2)$, for a point $p$ in "the punctured disk" $B_{a} \equiv B_{a}(i) \backslash\{i\}$ the fibre $\pi^{\prime-1}(p)$ consists of an orbit of $U(1)$ on which the function $\rho$ is positive and of another orbit on which it is negative, both being diffeomorphic to $U(1)$. The map $\pi^{\prime}$ factorizes as $C_{a} \stackrel{\pi_{1}}{\rightarrow} C_{a}^{\prime} \stackrel{\pi_{2}}{\rightarrow} B_{a}(i)$ where the quotient space $C_{a}^{\prime}=C_{a} / U(1)$ is diffcomorphic to ]$-a, a\left[\times S^{2}\right.$. Since the function $\rho$ on $C_{a}$ is invariant under the action of $U(1)$, it induces 
a function on $C_{a}^{\prime}$ which will also be denoted by $\rho$. For a fixed value of $\rho$ the map $\pi_{1}$ is the Hopf fibration realized by

$$
S U(2, C) \ni t \rightarrow \sqrt{2} \sigma^{k}{ }_{a b} t^{a}{ }_{0} t^{b}{ }_{1} \in S^{2} \subset \mathbb{R}^{3} .
$$

The set $\pi_{2}^{-1}\left(B_{a}\right)$ consists of a component $C_{a}^{\prime+}$ on which $\rho>0$ and a component $C_{a}^{\prime-}$ on which $\rho<0$. Each component is mapped diffeomorphically onto the punctured disk by $\pi_{2}$. Using $\pi_{2}$ to identify $C_{a}^{\prime+}$ with $B_{a}$ we obtain an extension $S^{*}$ of $\tilde{S}$. The point $i$ has been replaced here by the set $\pi_{1}\left(I^{0}\right)=\pi_{2}^{-1}(i)$ diffeomorphic to $S^{2}$, to which is glued the set $C_{a}^{\prime}$. Of course, from the point of view of the metric $h$ the set $\pi_{1}\left(I^{0}\right)$ still corresponds to a point and the function $\rho$ defined near it on $\tilde{S}$ is the geodesic distance from that point. Nevertheless, the initial value problem considered later will essentially be given an extension of the original initial hypersurface $\tilde{S}$ which is similar to $S^{*}$

Close to $i$ various properties of the data are analysed conveniently in terms of the $U(1)-$ bundle space $C_{a}$. The pull backs to $C_{a}$ of the connection form and the solder form on $S U(S)$ are analytic I-forms on $C_{a}$ which will be denoted again by $\check{\omega}^{a}{ }_{b}$ and $\sigma^{a b}$, respectively. Any smooth spinor field $\xi$ on an open subset $U$ of $B_{a}(i)$ is represented on the subset $\pi^{\prime}-1(U)$ of $C_{a}$ in a natural way by a smooth "spinor-valued function" $\xi_{a_{1} \ldots . . . a_{k}}$ which is given at $\delta \in \pi^{\prime-1}(U)$ by the components of $\xi$ in the frame defined by $\delta$. We shall refer to this function as to the "lift" of $\xi$.

The structure equations on $S U(S)$ imply on $C_{a}$ the equations

$$
\begin{aligned}
& \mathrm{d} \sigma^{a b}=-\check{\omega}^{a}{ }_{e} \wedge \sigma^{e b}-\check{\omega}^{b}{ }_{e} \wedge \sigma^{a e}, \\
& \mathrm{~d} \check{\omega}^{a}{ }_{b}=-\check{\omega}^{a}{ }_{e} \wedge \check{\omega}^{e}{ }_{b}+\check{\Omega}^{a}{ }_{b}
\end{aligned}
$$

with

$$
\check{\Omega}_{b}^{a}=\frac{1}{2} r^{a}{ }_{b c d e f} \sigma^{c d} \wedge \sigma^{e f}
$$

denoting the curvature form determined by the curvature spinor $r_{a b c d e f}$. It holds

$$
r_{a b c d e f}=\left(\frac{1}{2} s_{a b c c}-\frac{1}{12} r h_{a b c e}\right) \epsilon_{d f}+\left(\frac{1}{2} s_{a b d f}-\frac{1}{12} r h_{a b d f}\right) \epsilon_{c e},
$$

where $s_{a b c e}=s_{(a b c e)}$ is the trace-free part of the Ricci tensor of $h$ and $r$ its Ricci scalar. The curvature tensor of $h$ is given by

$$
r_{a g b h c d e f}=-r_{a b c d e f} \epsilon_{g h}-r_{g h c d e f} \epsilon_{a b}
$$

and the Bianchi identity reads

$$
D^{a b} s_{a b c d}=\frac{1}{6} D_{c d} r .
$$

We use $t \in S U(2, C)$ and $x^{1} \equiv \rho$ as coordinates on $C_{a}$. The vector field $\check{H}$ tangent to $C_{a}$ then takes the form $\sqrt{2} \breve{H}=\partial_{\rho}$. Further vector fields are defined on $C_{a}$ as follows. Consider the basis

$$
u_{1}=\frac{1}{2}\left(\begin{array}{cc}
0 & \mathrm{i} \\
\mathrm{i} & 0
\end{array}\right), \quad u_{2}=\frac{1}{2}\left(\begin{array}{cc}
0 & -1 \\
1 & 0
\end{array}\right), \quad u_{3}=\frac{1}{2}\left(\begin{array}{cc}
\mathrm{i} & 0 \\
0 & -\mathrm{i}
\end{array}\right)
$$


of the Lie algebra $s u(2, C)$, were $u_{3}$ is the generator of the group $U(1)$. We denote by $Z_{u_{i}}, i=0,1,2$, the Killing vector fields generated on $S U(S)$ by $u_{i}$ and the action of $S U\left(2, C^{\prime}\right)$. These fields are tangent to $I^{0}$ where we set

$$
X_{+}=-\left(Z_{u_{2}}+\mathrm{i} Z_{u_{1}}\right), \quad X_{-}=-\left(Z_{u_{2}}-\mathrm{i} Z_{u_{1}}\right), \quad X=-2 \mathrm{i} Z_{u_{3}} .
$$

These vector fields are extended to $C_{a}$ by the requirements

$$
[\check{H}, X]=0, \quad\left[\check{H}, X_{+}\right]=0, \quad\left[\check{H}, X_{-}\right]=0 .
$$

The analytic vector fields $\check{H}, X, X_{+}, X_{-}$constitute a frame field on $C_{a}$. It follows immediately from their definition that we also have

$$
\left[X, X_{+}\right]=2 X_{+}, \quad\left[X, X_{-}\right]=-2 X_{-}, \quad\left[X_{+}, X_{-}\right]=-X .
$$

For $p$ in the punctured disk $B_{a}$ the vector field $i X$ is tangent to the fibres $\pi^{\prime-1}(p)$. The complex vector fields $X_{+}, X_{-}$are complex conjugates of each other such that $\overline{X_{-} W}=$ $X_{+} W$ for any real-valued function $W$.

The definitions yield immediately

$$
\left\langle\sigma^{a b}, X\right\rangle=0, \quad\left\langle\check{\omega}^{a}{ }_{b}, X\right\rangle=\epsilon_{0}{ }^{a} \epsilon_{b}{ }^{0}-\epsilon_{1}{ }^{a} \epsilon_{b}{ }^{1} .
$$

By considerations similar to those used in [9] we find

$$
\begin{aligned}
& \left\langle\sigma^{a b}, X_{+}\right\rangle=\rho \sqrt{2} \epsilon_{0}{ }^{a} \epsilon_{0}{ }^{b}+\mathrm{O}\left(\rho^{2}\right), \quad\left\langle\check{\omega}^{a}{ }_{b}, X_{+}\right\rangle=\epsilon_{0}{ }^{a} \epsilon_{b}{ }^{1}+\mathrm{O}\left(\rho^{2}\right), \\
& \left\langle\sigma^{a b}, X_{-}\right\rangle=-\rho \sqrt{2} \epsilon_{1}{ }^{a} \epsilon_{1}{ }^{b}+\mathrm{O}\left(\rho^{2}\right), \quad\left\langle\check{\omega}^{a}{ }_{b}, X_{-}\right\rangle=-\epsilon_{1}{ }^{a} \epsilon_{b}{ }^{0}+\mathrm{O}\left(\rho^{2}\right)
\end{aligned}
$$

as $\rho \rightarrow 0$. It follows that for $p$ in the punctured disk the projections of $\breve{H}, X_{+}, X_{-}$span the tangent space at $p$.

We define furthermore vector fields $c_{a b}=c_{(a b)}$ on $C_{a} \backslash I^{0}$ by the requirements

$$
\left\langle\sigma^{a b}, c_{c d}\right\rangle=\epsilon_{(c}{ }^{a} \epsilon_{d)}^{b}, \quad c_{c d}=c^{1}{ }_{c d} \partial_{\rho}+c^{+}{ }_{c d} X_{+}+c^{-}{ }_{c d} X_{-} .
$$

The first condition implies $T_{\delta}\left(\pi^{\prime}\right) c_{a b}=\delta_{(a} \tau_{b} b^{\prime} \bar{\delta}_{b^{\prime}}$ for $\delta \in C_{a} \backslash I^{0}$. The second condition is imposed to ensure that the vector fields do not pick up a component in the direction of $X$. Using the properties of the solder and the connection form discussed above we find

$$
c_{a b}^{1}=x_{a b}+\check{c}_{a b}^{1}, \quad c^{+}{ }_{a b}=\frac{1}{\rho} z_{a b}+\check{c}^{+} a b, \quad c^{-}{ }_{a b}=\frac{1}{\rho} y_{a b}+\check{c}^{-} a b,
$$

where we have on the right-hand sides

$$
x_{a b} \equiv \sqrt{2} \epsilon_{(a}{ }^{0} \epsilon_{b)}{ }^{1}, \quad y_{a b} \equiv-\frac{1}{\sqrt{2}} \epsilon_{a}{ }^{1} \epsilon_{b}{ }^{1}, \quad z_{a b} \equiv \frac{1}{\sqrt{2}} \epsilon_{a}{ }^{0} \epsilon_{b}{ }^{0}
$$

and analytic functions satisfying

$$
\check{c}_{a b}^{\alpha}=\mathrm{O}(\rho), \quad \check{c}_{01}^{\alpha}=0, \quad \alpha=1,+,-
$$


Here the second equation is obtained because we have by construction $c_{01}=c_{10}=\check{H}$. It will be seen later as a consequence of the structure equations that

$$
\check{c}_{a b}^{l}=0 .
$$

We have connection coefficients

$$
\gamma_{c d}{ }^{a}{ }_{b} \equiv\left\langle\check{\omega}^{a}{ }_{b}, c_{c d}\right\rangle=\frac{1}{\rho} \gamma_{c d}^{*}{ }^{a}{ }_{b}+\check{\gamma}_{c d}{ }^{a}{ }_{b}
$$

with

$$
\gamma_{a b c d}^{*}=\frac{1}{2}\left(\epsilon_{a c} x_{b d}+\epsilon_{b d} x_{a c}\right), \quad \check{\gamma}_{01 c d}=0, \quad \check{\gamma}_{a b c d}=\mathrm{O}(\rho) .
$$

The analyticity of the solder form and of the vector fields $\breve{H}, X_{+}, X_{-}$entails that the vector fields $\rho c_{c d}$ whence the functions

$$
\rho \gamma_{c d}^{a}{ }_{b}, \quad c_{c d}^{1}, \quad \rho c^{+}{ }_{c d}, \quad \rho c^{-} c d
$$

extend to analytic fields on $C_{a}$.

A smooth function $F$ on an open subset of $C_{a}$ is said to have spin weight $s$ if

$$
X(F)=2 s F
$$

on this set with $2 s$ an integer. The construction of $C_{a}$ entails that any spinor-valued function on it has a well-defined spin weight.

The evaluation of the structure equations on the vector fields $c_{a b}$ and $X$ gives equations which show that the functions $c^{\alpha} a b, \gamma_{a b c d}$ have a well-defined spin weight. In view of (3.11), the evaluation of (3.5) on $X \wedge c_{c d}$ gives

$$
\left\langle\sigma^{a b},\left[X, c_{c d}\right]\right\rangle=\epsilon_{0}{ }^{a} \epsilon_{(c}{ }^{0} \epsilon_{d)}{ }^{b}-\epsilon_{1}{ }^{a} \epsilon_{(c}{ }^{1} \epsilon_{d)}{ }^{b}+\epsilon_{0}{ }^{b} \epsilon_{(c}{ }^{0} \epsilon_{d)}{ }^{a}-\epsilon_{1}{ }^{b} \epsilon_{(c}{ }^{1} \epsilon_{d)}{ }^{a} .
$$

Since it follows from (3.9) and (3.10) that the commutator of $X$ and $c_{a b}$ can be expanded in terms of the fields $c_{a b}$, this implies

$$
\left[X, c_{a b}\right]=2\left(\epsilon_{(a}^{0} c_{b) 0}-\epsilon_{(a}^{1} c_{b) 1}\right)
$$

Using (3.10) and the expansion of the frame vectors in (3.12), we arrive at the equations

$$
X c_{a b}^{1}=2(1-a-b) c_{a b}^{1}, \quad X c_{a b}^{ \pm}=2(1-( \pm 1)-a-b) c_{a b}^{ \pm},
$$

which determine the spin weights of the functions $c^{\alpha}{ }_{a b}$. The evaluation of (3.6) on $X \wedge c_{c d}$ gives

$$
X \gamma_{a b c d}=2(2-a-b-c-d) \gamma_{a b c d} .
$$

The evaluation of the structure equations on the vector fields $c_{a b}$ yields the essential content of the structure equations. By (3.12) the commutators of the vector fields $c_{a b}$ are of the form $\left[c_{a b}, c_{c d}\right]=A_{a b}{ }^{e f}{ }_{c d} c_{e f}+B_{a b c d} X$. Since $B_{a b c d}=c^{-}{ }_{a b} c^{+}{ }_{c d}-c^{-}{ }_{c d} c^{+}{ }_{a b}$, 
the vector fields $c_{a b}$ are nowhere tangent to a local section of $C_{a} \backslash I^{0}$ over the punctured disk. We get in particular

$$
\left[c_{01}, c_{c d}\right]=\frac{1}{\sqrt{2}}\left\{\partial_{\rho} c^{1} c d \partial_{\rho}+\partial_{\rho} c^{+}{ }_{c d} X_{+}+\partial_{\rho} c^{-}{ }_{c d} X_{-}\right\}
$$

The evaluation of (3.5) on $c_{c d} \wedge c_{e f}$ gives after contraction with $c_{a b}$ the equations

$$
A_{c d}{ }^{a b}{ }_{e f} c_{a b}=2\left\{\gamma_{c d}{ }^{a}{ }_{(e} \epsilon_{f)}{ }^{b}-\gamma_{e f}{ }^{a}{ }_{(c} \epsilon_{d)}{ }^{b}\right\} c_{a b} \text {. }
$$

These equations entail the radial equations

$$
\frac{1}{\sqrt{2}} \partial_{\rho} c^{\alpha}{ }_{a b}=-2 \gamma_{a b}{ }^{c}{ }_{(0} \epsilon_{1)}{ }^{d} c^{\alpha}{ }_{c d}, \quad \alpha=1,+,-,
$$

which are satisfied trivially if $a \neq b$. Observing (3.13) and (3.17), the non-trivial equations can be rewritten in the form

$$
\frac{1}{\sqrt{2}} \partial_{\rho}\left(\rho \check{c}_{a a}^{1}\right)=\check{\gamma}_{a a 00}\left(\rho \check{c}_{11}^{1}\right)-\check{\gamma}_{a a 11}\left(\rho \check{c}^{1}{ }_{00}\right) \text {, }
$$

which implies (3.16), and

$$
\begin{aligned}
& \frac{1}{\sqrt{2}} \partial_{\rho}\left(\rho \check{c}^{+}{ }_{a a}\right)=\check{\gamma}_{a a 00}\left(\rho \check{c}^{+}{ }_{11}\right)-\check{\gamma}_{a a 11}\left(\rho \check{c}^{+}{ }_{00}\right)-\frac{1}{\sqrt{2}} \check{\gamma}_{a a 11}, \\
& \frac{1}{\sqrt{2}} \partial_{\rho}\left(\rho \check{c}^{-}{ }_{a a}\right)=\check{\gamma}_{a a 00}\left(\rho \check{c}^{-}{ }_{11}\right)-\check{\gamma}_{a a 11}\left(\rho \check{c}^{-}{ }_{00}\right)-\frac{1}{\sqrt{2}} \check{\gamma}_{a a 00},
\end{aligned}
$$

which contain the non-trivial content of the radial equations. The evaluation of (3.6) on $c_{c d} \wedge c_{\text {ef }}$ gives the equations

$$
\begin{aligned}
& c_{c d}\left(\gamma_{e f}{ }^{a}{ }_{b}\right)-c_{e f}\left(\gamma_{c d}{ }^{a} b\right)+\gamma_{c d}{ }^{a}{ }_{h} \gamma_{e f}{ }^{h}{ }_{b}-\gamma_{e f}{ }^{a}{ }_{h} \gamma_{c d}{ }^{h}{ }_{b} \\
& \left.-2 \gamma_{g h}{ }^{a}{ }_{b}\left\{\gamma_{c d}{ }^{g}{ }_{(e} \epsilon_{f}\right)^{h}-\gamma_{e f}{ }^{g}{ }_{(c} \epsilon_{d)}{ }^{h}\right\} \\
& \quad-\left\{c^{-}{ }_{c d} c^{+}{ }_{e f}-c^{-}{ }_{e f} c^{+}{ }_{c d}\right\}\left(\epsilon_{0}{ }^{a} \epsilon_{b}{ }^{0}-\epsilon_{1}{ }^{a} \epsilon_{b}{ }^{l}\right)=r^{a} b c d e f .
\end{aligned}
$$

They entail the radial equations

$$
\begin{aligned}
\frac{1}{\sqrt{2}} \partial_{\rho} \gamma_{e f a b}= & -2 \gamma_{g h a b} \gamma_{e f}{ }^{g}{ }_{(0} \epsilon_{1)}{ }^{h} \\
& +\frac{1}{2}\left\{\left(s_{a b 0 e}-\frac{r}{6} h_{a b 0 e}\right) \epsilon_{1 f}+\left(s_{a b 1 f}-\frac{r}{6} h_{a b 1 f}\right) \epsilon_{0 e}\right\},
\end{aligned}
$$

which are satisfied trivially if $a \neq b$. Observing (3.13) and (3.17), the non-trivial equations can be rewritten in the form

$$
\begin{aligned}
& \frac{1}{\sqrt{2}}\left\{\partial_{\rho} \check{\gamma}_{00 c d}+\frac{1}{\rho}\left(\epsilon_{c}{ }^{0} \epsilon_{d}{ }^{0} \check{\gamma}_{0000}+\epsilon_{c}{ }^{1} \epsilon_{d}{ }^{1} \check{\gamma}_{0011}+\check{\gamma}_{00 c d}\right)\right\} \\
& =\check{\gamma}_{0000} \check{\gamma}_{11 c d}-\check{\gamma}_{0011} \check{\gamma}_{00 c d}-\frac{1}{2} s_{00 c d}+\frac{1}{12} r \epsilon_{\mathrm{c}}{ }^{1} \epsilon_{d}{ }^{1} \text {, } \\
& \frac{1}{\sqrt{2}}\left\{\partial_{\rho} \check{\gamma}_{11 c d}+\frac{1}{\rho}\left(\epsilon_{c}{ }^{0} \epsilon_{d}{ }^{0} \check{\gamma}_{1100}+\epsilon_{c}{ }^{1} \epsilon_{d}{ }^{1} \check{\gamma}_{1111}+\check{\gamma}_{11 c d}\right)\right\}
\end{aligned}
$$

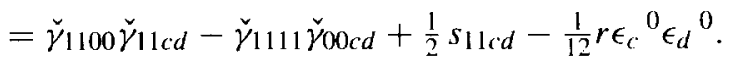


As a consequence of these equations we have

$$
\frac{1}{\sqrt{2}} \dot{\partial}_{\rho}\left\{\rho^{2}\left(\check{\gamma}_{1100}+\check{\gamma}_{0011}\right)\right\}=\left\{\rho^{2}\left(\check{\gamma}_{1100}+\check{\gamma}_{0011}\right)\right\}\left(\check{\gamma}_{1100}-\check{\gamma}_{0011}\right),
$$

which implies

$$
\check{\gamma}_{1100}=-\check{\gamma}_{0011} \text {. }
$$

The unknowns in the radial equations are not independent of each other. The vector fields $c_{a b}$ satisfy the reality condition $\left(c_{a b}\right)^{+} \equiv \tau_{a}{ }^{a^{\prime}} \tau_{a}{ }^{a^{\prime}} \bar{c}_{a^{\prime} b^{\prime}}=-c_{a b}$, the connection coefficients satisfy $\left(\gamma_{a b c d}\right)^{+}=-\gamma_{a b c d}$, and we have $\left(s_{a b c d}\right)^{+}=s_{a b c d}$.

Covariant differentiation is expressed on $C_{a} \backslash I^{0}$ by the "usual" rules. If $F$ denotes the lift of a smooth function $f$ on $B_{a}$, the covariant differential $D f$ is represented on $C_{a} \backslash I^{0}$ by the invariant function $D_{a b} f=c_{a b}(F)$. In the following we shall use the same symbol for a function and its lift. Let $\mu_{a b}$ be the invariant function representing a spatial spinor ficld $\mu$ on $B_{a}$. To derive an cxpression for the invariant function representing the covariant differential $D \mu$ consider $C_{a}$ as being embedded into $S U(S)$ and denote at $\delta \in C_{a} \backslash I^{0}$ the horizontal lift of $T_{\delta}(\pi) c_{a b}$ with respect to $\breve{\omega}^{a}{ }_{b}$ (which is not necessarily tangent to $C_{a} \backslash I^{0}$ ) by $c_{a b}^{\prime}$. The invariant function implied by $D \mu$ is then given on $C_{a} \backslash I^{0}$ by

$$
D_{a b} \mu_{c d}=c_{a b}^{\prime}\left(\mu_{c d}\right)=c_{a b}\left(\mu_{c d}\right)-\gamma_{a b}{ }^{e}{ }_{c} \mu_{e d}-\gamma_{a b}{ }^{e}{ }_{d} \mu_{c e} .
$$

Analogous formulas are obtained for covariant differentials of spinor fields of higher valence.

\section{2. $C_{a}$ versus $B_{a}(i)$}

We note some relations between $C_{a}$ and $B_{a}(i)$ and introduce notation needed later on. Let $x^{\alpha}$ be the normal coordinates on $B_{a}(i)$ centred at $i$ and based on the orthonormal frame

$$
c_{j}^{*}=\sigma_{j}^{a b} c_{a b}^{*} \equiv \sigma_{j}^{a b} \delta_{a}^{*} \delta_{b}^{*}
$$

at $i$ and let $V=x^{\beta} \partial / \partial x^{\beta}$ be the radial vector field, which is tangent to the geodesics through $i$ and satisfies $h(V, V)=-|x|^{2}$. Denote the spin frame on $B_{a}(i)$ which coincides with $\delta^{*}$ at $i$ and is parallely transported in the direction of $V$ again by $\delta^{*}=\delta^{*}\left(x^{\alpha}\right)$, and denote the frame field on $B_{a}(i)$ which is parallely transported along the geodesics through $i$ and coincides with $c_{j}^{*}$ at $i$ again by $c_{j}^{*}$ such that (3.26) holds everywhere on $B_{a}(i)$. The local expression of the projection $\pi^{\prime}$ is then given by

$$
\pi^{\prime}:(\rho, t) \rightarrow x^{\alpha}(\rho, t)=\rho \sqrt{2} c_{c d}^{* \alpha}(i) t^{c}{ }_{0} t^{d}{ }_{1}=\rho \sqrt{2} \delta^{\alpha}{ }_{j} \sigma^{j}{ }_{c d} t^{c}{ }_{0} t^{d}{ }_{1} .
$$

Thus the pull back of the function $|x|$ by $\pi^{\prime}$ is $|\rho|$. We write

$$
V(q)=V^{b c} c_{b c}^{*}=|x| v^{b c} c_{b c}^{*}
$$

on $B_{a}(i)$ with coefficients $V^{a b}, v^{a b}$ satisfying $V^{c d} V_{c d}=-|x|^{2}, v^{c d} v_{c d}=-1$, respectively. The latter are given by

$$
v^{a b}\left(x^{\alpha}(\rho, t)\right)=\sqrt{2} t_{0}^{(a} t_{1}^{b)}{ }_{1} .
$$


The pull back of the function $\Gamma$ by $\pi^{\prime}$ will be denoted again by $\Gamma$ such that we have $\Gamma=\rho^{2}$ on $C_{a}$. The map $\pi^{\prime}$ projects the frame $c_{a b}$ at the point $(\rho, t), \rho \neq 0$, onto the frame

$$
T\left(\pi^{\prime}\right) c_{a b}=\delta_{a}(\rho, t) \delta_{b}(\rho, t)=\delta_{c}^{*}\left(x^{\alpha}(\rho, t)\right) t_{a}^{c} \delta_{d}^{*}\left(x^{\alpha}(\rho, t)\right) t^{d}{ }_{b} .
$$

\subsection{Normal expansions at $i$}

Since the singular fields which we will have to analyse are usually built from fields on $S$ which are analytic near $i$, we begin by recalling a certain type of expansion of analytic fields near $i$. We use the notation introduced above and write $D_{j}$ for the covariant derivative in the direction of $c_{j}^{*}$.

Suppose $T$ is an analytic tensor field of rank $(r, s)$ on $B_{a}(i)$ which has components $T^{* i_{1} \cdots i_{r}} j_{1} \ldots j_{s}$ with respect to the frame $c_{j}^{*}$. Since $D_{V} c_{j}^{*}=0$, we find for $x^{\alpha} \in B_{a}(i)$, $|t| \leq 1$,

$$
\begin{aligned}
& \frac{\mathrm{d}}{\mathrm{d} t}\left(T^{* i_{1} \cdots i_{r}}{ }_{j_{1} \cdots j_{\mathrm{s}}}\left(t x^{\alpha}\right)\right)=x^{\beta}\left(\frac{\partial}{\partial x^{\beta}} T^{* i_{1} \cdots i_{r}} j_{1} \cdots j_{s}\right)\left(t x^{\alpha}\right) \\
& =V^{l}\left(x^{\alpha}\right)\left(D_{l} T^{* i_{1} \cdots i_{r}}{ }_{j_{1} \cdots j_{s}}\right)\left(t x^{\alpha}\right) \text {. }
\end{aligned}
$$

Observing that such formulae also hold for the covariant differentials of $T$, we get by induction

$$
\begin{aligned}
& \frac{\mathrm{d}^{p}}{\mathrm{~d} t^{p}} T^{* i_{1} \cdots i_{r}}{ }_{j_{1} \cdots j_{s}}\left(t x^{\alpha}\right)=V^{l_{p}} \ldots V^{l_{1}} D_{l_{p}} \cdots D_{l_{1}}\left(T_{j_{1} \cdots j_{s}}^{* i_{1} \cdots i_{r}}\right)\left(t x^{\alpha}\right), \\
& \quad p=0,1,2, \ldots
\end{aligned}
$$

Evaluating the Taylor expansion

$$
T_{j_{1} \cdots j_{s}}^{* i_{1} \cdots i_{r}}\left(t x^{\alpha}\right)=\left.\sum_{p=0}^{\infty} t^{p} \frac{1}{p !} \frac{\mathrm{d}^{p}}{\mathrm{~d} t^{p}}\left(T^{* i_{1} \cdots i_{r}} j_{1} \cdots j_{s}\left(t x^{\alpha}\right)\right)\right|_{t=0}
$$

at $t=1$, we get

$$
\begin{aligned}
& T_{j_{1} \cdots j_{s}}^{* i_{1} \cdots i_{r}}(q)=\sum_{p=0}^{\infty} \frac{1}{p !} V^{l_{p}}(q) \cdots V^{l_{1}}(q) D_{l_{p}} \cdots D_{l_{1}} T^{* i_{1} \cdots i_{r}}{ }_{j_{1} \cdots j_{s}}(i), \\
& q \in B_{a}(i) .
\end{aligned}
$$

Since only covariant operations occur on the right hand side, the final result does neither depend on the normal coordinates $x^{\alpha}$ nor on the frame $\mathrm{e}_{k}$ and it can be written (with the bracket indicating the obvious contraction) in the short form

$$
T(q)=\sum_{p=0}^{\infty} \frac{1}{p !}\left(\left\langle V(q)^{\otimes^{p}}, D^{p}\right\rangle T\right)(i) .
$$

Analogous expansions are obtained for analytic spinor fields. Let $\xi_{a_{1} \cdots a_{l}}^{*}$ be the components of the symmetric analytic spinor field $\xi$ on $B_{a}(i)$ with respect to the spin frame $\delta^{*}\left(x^{\alpha}\right)$. By similar arguments as above we find the expansion 


$$
\xi_{a_{1} \cdots a_{l}}^{*}(q)=\sum_{p=0}^{p=\infty}|x|^{p} \frac{1}{p !} v^{b_{p} c_{p}} \ldots v^{b_{1} c_{1}} D_{b_{p} c_{p}} \cdots D_{b_{1} c_{1}} \xi_{a_{1} \cdots a_{l}}^{*}
$$

with $v^{a b}=v^{a b}(q), q \in B_{a}(i)$, and the derivatives of the spinor field evaluated at the point $i$. Since the index pairs $b_{j} c_{j}, j=1,2, \ldots, p$, are all transvected with the same spinor $v^{c d}$, the derivatives in (3.30) can be replaced by the symmetrized (in the pairs on indices) derivatives. If in the symmetrized derivatives a contraction of indices $c_{j}, c_{k}, j \neq k$, is performed, the resulting expression is antisymmetric in the indices $b_{j}, b_{k}$. Thus, decomposing into irreducible parts (with respect to the indices $b_{p}, \ldots, c_{1}$ ) yields in each summand of $(3.30)$ with $p \geq 2$ an expansion

$$
\begin{aligned}
v^{b_{p} c_{p}} \cdots v^{b_{1} c_{1}} D_{b_{p} c_{p}} & \cdots D_{b_{1} c_{1}} \xi_{a_{1} \cdots a_{l}}^{*} \\
=v^{b_{p} c_{p}} \cdots v^{b_{1} c_{1}} & \xi_{p, 0 ; b_{p} c_{p} \cdots b_{1} c_{1} a_{1} \ldots a_{l}}^{*} \\
& \left.+\xi_{p, 1 ; b_{p} c_{p} \cdots b_{3} c_{3} a_{1} \cdots a_{l}}^{*} h_{b_{1} c_{1} b_{2} c_{2}}+\cdots\right)
\end{aligned}
$$

where the dots indicate terms (vanishing for $p \leq 3$ ) which are at least quadratic in the metric tensor $h_{a b c d}$. The spinors satisfy the symmetry condition

$$
\xi_{p, i: b_{p} c_{p} \cdots b_{2 i+1} c_{2 i+1} a_{1} \cdots a_{l}}^{*}=\xi_{p, i:\left(b_{p} c_{p} \cdots b_{2 i+1} c_{2 i+1}\right)\left(a_{1} \cdots a_{l}\right)}^{*} \quad i=0, \ldots,\left[\frac{1}{2} p\right]
$$

with $\left[\frac{1}{2} p\right]=\frac{1}{2} p$ if $p$ is even, $\left[\frac{1}{2} p\right]=\frac{1}{2}(p-1)$ if $p$ is odd. In particular

$$
\begin{aligned}
& \xi_{p, 0 ; b_{p} c_{p} \cdots b_{1} c_{1} a_{1} \cdots a_{l}}^{*}=D_{\left(b_{p} c_{p}\right.} \cdots D_{\left.b_{1} c_{1}\right)} \xi_{a_{1} \cdots a_{l}}^{*}, \\
& \xi_{p, 1 ; b_{p} c_{p} \cdots b_{3} c_{3} a_{1} \cdots a_{l}}^{*} \\
& \quad=\frac{1}{2 p-1} \sum_{1 \leq k<h \leq p} D_{\left(b_{p} c_{p}\right.} \cdots D_{|e f|} \cdots D^{e f} \cdots D_{\left.b_{3} c_{3}\right)} \xi_{a_{1} \cdots a_{l}}^{*},
\end{aligned}
$$

where the dots on the right-hand side of the last equation indicate operators of the form $D_{b_{j} c_{j}}$ and where $D_{e f}, D^{e f}$ are assumed to stand in the $k$ th and $h$ th position respectively.

To obtain the complete decomposition of the coefficients of (3.30) into irreducible parts one finally has to observe the expansion

$$
\begin{aligned}
& \xi_{p, i ; b_{p} c_{p} \cdots b_{2 i+1} c_{2 i+1}}^{a_{1} \cdots a_{l}} \\
& =\xi_{p, i: b_{p} c_{p} \cdots b_{2 i+1} c_{2 i+1}}^{0}{ }^{a_{1} \cdots a_{l}}+\xi_{p, i:\left(b_{p} c_{p} \cdots b_{2 i+1}\right.}^{1}{ }^{\left(a_{1} \cdots a_{l-1}\right.} \epsilon_{\left.c_{2 i+1}\right)}{ }^{\left.a_{l}\right)} \\
& +\xi_{p, i ;\left(b_{p} c_{p} \cdots c_{2 i+3}\right.}^{2}\left(a_{1} \cdots a_{l-2} \epsilon_{b_{2 i+1}} a^{a_{l-1}} \epsilon_{\left.c_{2 i+1}\right)}\right)^{\left.a_{l}\right)}+\cdots
\end{aligned}
$$

with totally symmetric spinors satisfying

$$
\begin{aligned}
& \xi_{p, i ; b_{p} c_{p} \cdots b_{2 i+1} c_{2 i+1} a_{1} \cdots a_{l}}^{0}=\xi_{p, i ;\left(b_{p} c_{p} \cdots b_{2 i+1} c_{2 i+1} a_{1} \cdots a_{l}\right)}^{*}, \\
& \xi_{p, i ; b_{p} c_{p} \cdots b_{2 i+1} a_{1} \cdots a_{l-1}}^{1}=c_{p, i, l}^{1} \xi_{p, i ;\left(b_{p} c_{p} \cdots b_{2 i+1}|e| a_{1} \cdots a_{l-1}\right)}^{*}, \ldots
\end{aligned}
$$

and positive real coefficients $c_{p, i, l}^{1}$, etc. 


\subsection{The functions $T_{m}{ }_{k}$}

If expression (3.30) of $\xi$ is lifted to $C_{a}$, we encounter a certain class of functions on $C_{a}$ associated with unitary representations $S U(2, C)$. The matrix elements of these representations are given by the complex-valued functions

$$
\begin{gathered}
S U(2, C) \ni t \rightarrow T_{m}{ }_{k}{ }_{k}(t)=\left(\begin{array}{c}
m \\
j
\end{array}\right)^{1 / 2}\left(\begin{array}{c}
m \\
k
\end{array}\right)^{1 / 2} t^{\left(b_{1}\right.}\left(a_{1} \cdots t^{\left.b_{m}\right)_{j}} a_{m}\right)_{k}, \\
T_{0}{ }^{0}{ }_{0}(t)=1, \quad j, k=0, \ldots, m, \quad m=1,2.3, \ldots
\end{gathered}
$$

where, as in the following, setting a string of indices into brackets with a lower index $k$ is meant to indicate that the indices are symmetrized and then $k$ of them are set equal to 1 while the remaining ones are set equal to 0 . We state a few properties of these functions which follow directly from their definition; more details can be found in [9]. The functions are real analytic on $S U(2, C)$, the representation is given by

$$
S U(2, C) \ni t \rightarrow T_{m}(t)=\left(T_{m}{ }_{k}(t)\right) \in S U(m+1) .
$$

and the functions $\sqrt{m+1} T_{m}{ }_{k}(t)$ form a complete orthonormal set in the Hilbert space $L^{2}(\mu, S U(2, C))$ where $\mu$ denotes the normalized Haar measure on $S U(2, C)$. In particular. any real analytic complex-valued function $f$ on $S U(2, C)$ admits an expansion

$$
f(t)=\sum_{m=0}^{\infty} \sum_{j=0}^{m} \sum_{k=0}^{m} f_{m, k, j} T_{m}^{k}{ }_{j}(t)
$$

with complex coefficients $f_{m, k, j}$ which vanish rapidly as $m \rightarrow \infty$. To be able to take care of reality conditions, we note that under complex conjugation the functions transform as

$$
\overline{T_{m}{ }_{k}(t)}=(-1)^{j+k} T_{m}{ }^{m-j}{ }_{m-k}(t), \quad t \in S U(2 . C) .
$$

We denote by $Z_{u_{i}}, i=0,1,2$, the left invariant vector fields on $S U(2, C)$ generated by the $u_{i}$ given in (3.8) and set

$$
X_{+}=-\left(Z_{u_{2}}+\mathrm{i} Z_{u_{1}}\right), \quad X_{-}=-\left(Z_{u_{2}}-\mathrm{i} Z_{u_{1}}\right), \quad X=-2 \mathrm{i} Z_{u_{3}} .
$$

It follows then for $0 \leq k, j \leq m, m=0,1,2, \ldots$ that

$$
\begin{aligned}
& X T_{m}{ }^{k}{ }_{j}=(m-2 j) T_{m}{ }^{k}{ }_{j}, \quad X_{+} T_{m}{ }^{k}{ }_{j}=\beta_{m, j} T_{m}{ }^{k}{ }_{j-1}, \\
& X-T_{m}{ }^{k}{ }_{j}=-\beta_{m, j+1} T_{m}{ }^{k}{ }_{j+1}
\end{aligned}
$$

with

$$
\beta_{m, j}=\{j(m-j+1)\}^{1 / 2} .
$$

From this ensues that for a function $f$ with integer or half integer spin weight $s$, i.e.

$$
X f=2 s f
$$


the expansion above reduces to the form

$$
f=\sum_{m \geq|2 s|} \sum_{k=0}^{m} f_{m, k} T_{m}{ }^{k}{ }_{m / 2-s},
$$

where $m$ takes even values if $s$ is an integer and odd values if $s$ is a half integer.

The products of two functions of the type above can be expanded in the form

$$
\begin{aligned}
T_{m}{ }_{k}{ }_{k} T_{n}{ }^{j}= & \sum_{p=0}^{\mu} D(m, i, k ; n, j, l ; m+n-2 p, i+j-p, k+l-p) \\
& \times T_{m+n-2 p}{ }^{i+j-p} k+l-p
\end{aligned}
$$

with $\mu=\min \{m, n, i+j, k+l\}$ and coefficients $D(m, i, k ; n, j, l ; w, r, s)$ which are products

$$
D(m, i, k ; n, j, l ; w, r, s)=C(m, i ; n, j ; w, r) \overline{C(m, k ; n, l ; w, s)}
$$

of Clebsch-Gordan coefficients $C(m, i ; n, j ; w, r)$. Since the latter satisfy

$$
\sum_{i, j}|C(m, i ; n, j ; w, r)|^{2}=1,
$$

we have $|D(m, i, k ; n, j, l ; w, r, s)| \leq 1$ for all admissible values of the indices.

We note the following special case of the expansion formula above:

$$
\begin{aligned}
& T_{2 m}{ }^{i}{ }_{k} T_{2 n}{ }^{j} l \\
&= \sum_{q=q_{0}}^{m+n} D(2 m, i, k ; 2 n, j, l ; 2 q, i+j+q-m-n, k+l+q-m-n) \\
& \times T_{2 q}{ }^{i+j+q-m-n} k+l+q-m-n
\end{aligned}
$$

with

$$
q_{0}=\max \{|m-n|, m+n-i-j, m+n-k-l\} .
$$

For an even more specialized case we shall need later the following information on the coefficient of the highest order term in this expansion.

Lemma 3.1. If $m$ is an integer and $n, p, k, k^{\prime}, j$ are non-negative integers such that $p-n \geq$ $0, n+m \geq 2, p+m \geq 2,0 \leq k \leq 2(p-n), 0 \leq k^{\prime} \leq 2(n+m)$, then in the expansion

$$
\begin{aligned}
& T_{2(p-n)}{ }^{k}{ }_{p-n} T_{2(n+m)}{ }^{k^{\prime}}{ }_{n+m-2+j} \\
& \quad=D(j) T_{2(p+m)}{ }^{k+k^{\prime}}{ }_{p+m-2+j}+\text { "terms of lower order" }
\end{aligned}
$$

the coefficients $D(j)$ (suppressing all the other indices since we are interested here only in the dependence on $j$ ) do not vanish and satisfy $D(0)=D(4)$.

Applying $(p+m-2+j)$-times the operator $X_{+}$to the expansion above, all the lower order terms drop out and we get, observing the Leibniz rule, 


$$
\begin{aligned}
D(j) & \prod_{l=1}^{p+m-2+j} \beta_{2(p+m), l} T_{2(p+m)}{ }^{k+k^{\prime}} 0 \\
= & D(j) X_{+}^{p+m-2+j} T_{2(p+m)}{ }^{k+k^{\prime}} p+m-2+j \\
= & \left(\begin{array}{l}
p+m-2+j \\
n+m-2+j
\end{array}\right) \prod_{l=1}^{p-n} \beta_{2(p-n), l} \prod_{l=1}^{n+m-2+j} \beta_{2(n+m), l} T_{2(p-n)}{ }^{k}{ }_{0} T_{2(n+m)} k^{\prime}
\end{aligned}
$$

This equation implies $D(j) \neq 0$ and

$$
\frac{D(4)}{D(0)}=\frac{\left(\begin{array}{c}
p+m+2 \\
n+m+2
\end{array}\right) \prod_{l=n+m-1}^{n+m+2} \beta_{2(n+m), l}}{\left(\begin{array}{c}
p+m-2 \\
n+m-2
\end{array}\right) \prod_{l=p+m-1}^{n+m+2} \beta_{2(p+m), l}}=1 .
$$

The functions $T_{m}{ }_{k}{ }_{k}$ on $S U(2, C)$ induce functions on $C_{a}$ which are obtained as follows. The set $I^{0}$ is identified by its construction with the set $S U(2, C)$. Thus we can consider the functions $T_{m}{ }_{k}{ }_{k}$ as functions on $I^{0}$. We extend the functions $T_{m}{ }_{k}{ }_{k}$ to analytic functions on $C_{a}$ such that $\check{H} T_{m}{ }^{j}{ }_{k}=0$. By the same identification the vector fields $Z_{u_{i}}, X_{+}, X_{-}, X$ on $S U(2, C)$ are identified on $I^{0}$ with the vector fields introduced earlier for which we used the same notation. Relations (3.35) remain true on $C_{a}$.

\subsection{Normal expansions at $I^{0}$}

Any analytic function $f$ on $C_{a}$ with integer spin weight $s$ has an expansion of the type (3.36) with coefficients $f_{q, k}$ which are analytic functions of $\rho$. In the case of spin-valued functions arising from spinor fields on $B_{a}(i)$ the expansions are of a very specific kind. Suppose $\xi_{a_{1} \cdots a_{l}}$ is the spin-valued function on $C_{a}$ representing a symmetric analytic spinor field on $B_{a}(i)$ and denote by $\xi_{j}=\xi_{\left(a_{1} \cdots a_{l}\right)_{j}}, 0 \leq j \leq l$, its essential components. The function $\xi_{j}$ has spin weight $s=l / 2-j$ and a unique expansion of the form

$$
\xi_{j}=\sum_{p=0}^{\infty} \xi_{j . p} \rho^{p}
$$

with

$$
\xi_{j, p}=\sum_{m=\max \{|l-2 j|, l-2 p\}}^{2 p+l} \sum_{k=0}^{m} \xi_{j, p: m, k} T_{m}^{k}{ }_{(m-l) / 2+j}
$$

and complex coefficients $\xi_{j, p ; m, k}$. Here $m$ takes even values if $l$ is even and odd values otherwise. We shall refer to this type of expansion as to the "normal" expansion at $I^{0}$. Its form can be deduced by the abstract arguments given in [9]. To see how it relates to the spinor field $\xi$ on $B_{a}(i)$ it is more useful to derive it directly from (3.30). Expression (3.39) at the point $(\rho, t)$ is obtained from (3.30) by the following operations on Eq. (3.30):

(i) Perform the transition $\xi_{a_{1} \cdots a_{l}}^{*} \rightarrow \xi_{b_{1} \cdots b_{l}}^{*} t^{b_{1}} a_{1} \cdots t^{b_{l}} a_{l}$.

(ii) Replace $|x|$ by $\rho$ and the coefficients $v^{a b}$ by the right-hand side of (3.29). 
(iii) Decompose all spinor-valued coefficients into sums of products of symmetric coefficients with $\epsilon$ 's. Contractions of $\epsilon$ 's with pairs of $t$ 's yield factors 1 or 0 . The remaining expressions assume the form of expansions in terms of the functions $T_{m}{ }^{k} l$.

The coefficient of $\rho^{p}$ is then

$$
\begin{aligned}
& \left.(\sqrt{2})^{p} D_{b_{p} c_{p}} \cdots D_{b_{1} c_{1}} \xi_{\mathrm{e}_{1} \cdots \mathrm{e}_{l}}^{*} t^{b_{p}}{ }_{0} t^{c_{p}}{ }_{1} \cdots t^{b_{1}}{ }_{0} t^{c_{1}}{ }_{1} t^{\mathrm{e}_{1}}{ }_{\left(a_{1}\right.} \cdots t^{\mathrm{e}_{l}}{ }_{a_{l}}\right)_{j} \\
& =\sum_{m=\max \{|l-2 j|, l-2 p\}}^{2 p+l-2} \sum_{k=0}^{m} \xi_{j, p ; m, k} T_{m}{ }^{k}{ }_{(m-l) / 2+j} \\
& +(\sqrt{2})^{p} D_{\left(b_{p} c_{p}\right.} \cdots D_{b_{1} c_{1}} \xi_{\mathrm{e}_{1} \cdots \mathrm{e}_{l}}^{*} t^{b_{p}}{ }_{0} t^{c_{p}}{ }_{1} \cdots t_{0}^{b_{1}} t^{c_{1}} t_{1}^{\mathrm{e}_{1}}{ }_{\left(a_{1}\right.} \cdots t^{\mathrm{e}_{t}}{ }_{\left.a_{l}\right)_{j}} .
\end{aligned}
$$

The second term on the right-hand side, which is of order $q=2 p+l$ in the $t$ 's, has the expansion

$$
\begin{aligned}
& (\sqrt{2})^{p} D_{\left(b_{p} c_{p}\right.} \cdots D_{b_{1} c_{1}} \xi_{\left.\mathrm{e}_{1} \ldots \mathrm{e}_{l}\right)}^{*} t^{b_{p}}{ }_{0} t^{c_{p}}{ }_{1} \cdots t^{b_{1}}{ }_{0} t^{c_{1}} t^{\mathrm{e}_{l}}{ }_{\left(a_{1}\right.} \cdots t_{\left.a_{l}\right)_{j}}^{\mathrm{e}_{l}} \\
& =\sum_{k=0}^{2 p+l} \xi_{j, p ; 2 p+l, k} T_{2 p+l}^{k}{ }_{p+j}
\end{aligned}
$$

with

$$
\xi_{j, p ; 2 p+l, k}=(\sqrt{2})^{p}\left(\begin{array}{c}
2 p+l \\
k
\end{array}\right)^{1 / 2}\left(\begin{array}{c}
2 p+l \\
p+j
\end{array}\right)^{-1 / 2} D_{\left(b_{p} c_{p}\right.} \cdots D_{b_{1} c_{1}} \xi_{\left.\mathrm{e}_{1} \ldots \mathrm{e}_{l}\right)_{k}}^{*}
$$

Thus we find the symmetry

$$
\xi_{0, p ; 2 p+l, k}=\xi_{l, p ; 2 p+l, k},
$$

which will be important for us later.

We shall also be interested in (symmetric) systems of analytic functions $\xi_{a_{1} \cdots a_{2 r}}$ with "essential components" $\xi_{j}=\xi_{\left(a_{1} \cdots a_{2 r}\right)_{j}}, 0 \leq j \leq 2 r$, of spin weight $s=r-j$ which do not descend to analytic spinor fields on $B_{a}(i)$. In this case we have again expansions of the form (3.39) but (3.40) is now replaced by the more general expression

$$
\xi_{j, p}=\sum_{q=|r-j|}^{q(p)} \sum_{k=0}^{2 q} \xi_{j, p: 2 q, k} T_{2 q}^{k}{ }_{q-r+j}
$$

where for given $r$ we have a priori

$$
0 \leq|r-j| \leq q(p) \leq \infty .
$$

In the cases considered later $q(p)$ will he a finite integer for given positive integer $p$.

An expansion of the form (3.39) and (3.42), or simply an expansion coefficient like (3.42), will in the following be said to be of "expansion type $q(p)$ ". It will be understood here that terms for which $q(p)<0$ vanish. It should be noted that we do not require $q(p)$ to be the minimal function for which (3.42) is true.

Spin weights and expansion types obey the following rules under algebraic operations on functions. Suppose the functions $f, g$ have spin weights $s=2 r, s^{\prime}=2 r^{\prime}$, with $r, r^{\prime}$ integers, 
and are of expansion type $q(p), q^{\prime}(p)$, respectively. Then by (3.37) the product $f g$ has spin weight $2\left(r+r^{\prime}\right)$ and expansion type $q_{\times}(p)=\max _{0 \leq j \leq p}\left\{q^{\prime}(j)+q(p-j)\right\}$. If $s=s^{\prime}$ the sum $f+g$ has well-defined spin weight $s$ and expansion type $q_{+}(p)=\max \left\{q(p), q^{\prime}(p)\right\}$. Of course, it may happen that due to cancellations for certain functions smaller expansion types may he assigned to their sum or product. The control of the expansion types will be quite important for us later on and we shall use the rules above repeatedly without referring to them in each case.

In the case where $l=2 r$ and $m=2 q$ the reality condition $\xi^{+}=(-1)^{r} \xi$ reads

$$
\xi_{j}=(-1)^{j} \bar{\xi}_{l-j}
$$

or, in terms of the expansion coefficients.

$$
\xi_{j, p: 2 q, k}=(-1)^{r+q+k} \bar{\xi}_{l-j, p: 2 q, 2 q-k} .
$$

\section{Structure of data near space-like infinity}

In this section we shall investigate normal expansions of the initial data in some detail. The need for the specific information on the data derived in this section will become clear only when we study the evolution of the fields.

The following facts will be used repeatedly. The curvature tensor of $h$ is given in three dimensions by

$$
r_{m j l k}=h_{m l} l_{k j}-h_{m k} l_{l j}+h_{j k} l_{l m}-h_{j l} l_{k m}, \quad l_{i j}=s_{i j}+\frac{1}{12} r h_{i j},
$$

where $s_{j k}$ denotes the trace-free part of the Ricci tensor $r_{j k}$ of $h$ and $r$ denotes the Ricci scalar. Since we are dealing with problems of conformal geometry we shall need the Cotton tensor

$$
k_{p i j}=D_{j} l_{i p}-D_{i} l_{j p} \quad \text { resp. } \quad k_{i j}=-\frac{1}{2} k_{i k l} \epsilon_{j}{ }^{k l} .
$$

We have

$$
k_{i}{ }^{i}=0, \quad k_{i j}=k_{j i}, \quad D^{i} k_{i j}=0 .
$$

The space-spinor representation $k_{a b c d}$ ef of the Cotton tensor has decomposition

$$
k_{a b c d e f}=b_{a b c e} \epsilon_{d f}+b_{a b d f} \epsilon_{c e},
$$

where

$$
b_{a b c d} \equiv D^{h}{ }_{(a} s_{b c d) h}
$$

satisfies

$$
D^{n h} b_{a b c d}=0 .
$$

Under rescalings $h \rightarrow h^{\prime}=\phi^{2} h$ we have transformation laws

$$
k_{p i j}^{\prime}=k_{p i j}, \quad k_{i j}^{\prime}=\phi^{-1} k_{i j} . \quad b_{a b c d}^{\prime}=\phi^{-1} b_{a b c d} .
$$




\subsection{The expansion type of the rescaled Weyl tensor}

The rescaled conformal Weyl tensor is in a sense the most important unknown in the propagation equations. We shall analyse now its normal expansion at $I^{0}$.

Denote by $C_{a}^{+}$the subset of $C_{a}$ on which $\rho$ is positive. If we lift the function $\Omega$ to $C_{a}^{+}$ and consider it as a function of the coordinates $\rho, t$, it is an analytic function which can be extended analytically beyond the set $I^{0}$. We shall refer to analytic functions obtained by lifting to $C_{a}^{+}$and extending analytically through $I^{0}$ as to the "analytic lift to $C_{a}$ " of the given function on $B_{a}(i)$. Since we will consider this procedure only for a finite number of functions, we shall assume that the number $a$ has been chosen small enough for the analytic lifts to be defined an all of $C_{a}$.

We choose now a positive, analytic function $\kappa^{\prime}$ on $B_{a}(i)$ with $\kappa^{\prime}(i)=1$ and set $\kappa=|x| \kappa^{\prime}$. To state our first main result, we consider the analytic lifts of the tensor fields $\kappa^{3} \phi_{a b c d}$, $\kappa^{3} \phi_{a b c d}^{\prime}, \kappa^{3} \phi_{a b c d}^{W}$ on $B_{a}(i)$ which we denote by $\breve{\phi}_{a b c d}, \breve{\phi}_{a b c d}^{\prime}, \breve{\phi}_{a b c d}^{W}$, respectively. The following properties of the conformal Weyl tensor will be important for us.

Theorem 4.1. The analytic lifts $\breve{\phi}_{a b c d}, \breve{\phi}_{a b c d}^{\prime}, \breve{\phi}_{a b c d}^{W}$ on $C_{a}$ have expansion types $p$.

(i) The expansion coefficients $\breve{\phi}_{j, p, 2 q, k}^{W}$ of the functions $\breve{\phi}_{j}^{W}=\breve{\phi}_{(a b c d)_{j}}^{W}$ satisfy the symmetry condition

$$
\breve{\phi}_{0, p, 2 p, k}^{W}=\breve{\phi}_{4, p, 2 p, k}^{W}, \quad p=0,1,2, \ldots, k=0, \ldots, 2 p .
$$

(ii) The expansion coefficients $\breve{\phi}_{j, p, 2 q, k}^{\prime}$ of the functions $\breve{\phi}_{j}^{\prime}=\breve{\phi}_{(a b c d)_{j}}^{\prime}$ satisfy the antisymmetry condition

$$
\breve{\phi}_{0, p, 2 p, k}^{\prime}=-\breve{\phi}_{4, p, 2 p, k}^{\prime}, \quad p=0,1,2, \ldots, k=0, \ldots, 2 p .
$$

(iii) If and only if the Cotton tensor satisfies the condition

$$
D_{\left(a_{q} b_{q}\right.} \cdots D_{a_{1} b_{1}} b_{a b c d)}(i)=0, \quad q=0,1,2, \ldots
$$

we have

$$
\breve{\phi}_{j, p, 2 p, k}^{\prime}=0, \quad p=0,1,2, \ldots, k=0, \ldots, 2 p, j=0, \ldots, 4
$$

and the functions $\breve{\phi}_{j}^{\prime}$ have expansion type $p-1$.

It will be seen in Section 7 that property (4.6) is related to a certain type of non-smooth behaviour of the fields at the "sets $I^{ \pm}$where null infinity touches space-like infinity" (cf. Section 5). Condition (4.7) will be recognized then as a condition on the data which is implied by the requirement that the solution evolves smoothly through the sets $I^{ \pm}$.

Condition (4.7) has been observed before in a related though somewhat different context. In [10] it has been derived as a consequence of the requirement, called "radiativity condition", that the spinor field $\phi_{a b c d}^{\prime}$ has an analytic extension to $B_{a}(i)$. Answering a question which has been posed in [10], we shall show that (4.7) is in fact equivalent to the radiativity condition. 
Theorem 4.2. We consider the tensor field

$$
e_{i j}^{\prime}=D_{i} D_{j} \Omega^{\prime}-\frac{1}{3} h_{i j} D_{k} D^{k} \Omega^{\prime}+\Omega^{\prime} s_{i j}
$$

which is derived from the analytic function $\Omega^{\prime}=\Gamma / U^{2}$ on $B_{a}(i)$. The following conditions are equivalent:

(i) The Cotton tensor satisfies condition (4.7).

(ii) The complex analytic extension of the function $b_{k l} D^{k} \Omega^{\prime} D^{l} \Omega^{\prime}$, defined on the complex analytic extension of $\left(B_{a}(i), h\right)$, vanishes near $i$ on the complex cone $N$ which is generated by the null geodesics through $i$.

(iii) The field $c_{k l} \equiv \Omega^{\prime-1} e_{k l}^{\prime}$, i.e. the massless part of the Weyl tensor, extends to $i$ as a real analvtic function.

(iv) The field $d_{k l}^{\prime}=\Omega^{\prime-2} e_{k l}^{\prime}$, i.e. the massless part of the rescaled Weyl tensor, extends to $i$ as a real analytic function.

Remarks. Under conformal rescalings the complex null cone $N$, which coincides near $i$ with the set $\left\{\Omega^{\prime}=0\right\}$, is invariant and the function $b_{k l} D^{k} \Omega^{\prime} D^{\prime} \Omega^{\prime}$ transforms on $N$ like a conformal density. This entails that condition (ii) is conformally invariant and we see that (4.7) is in fact a condition on the free initial data. We note that also the other conditions of Theorem 4.1 are invariant under conformal rescalings.

The lift of a smooth spinor field of rank 4 being of expansion type $p+2$, multiplication by a factor $\kappa^{3}$ gives a field of expansion type $p-1$. Thus it follows as a simple consequence that the field $\breve{\phi}_{a b c d}^{\prime}$ has expansion type $p-1$ if the conditions of Theorem 4.2 are satisfied.

It should be noted that the argument by which we will be led in Section 7 to consider (4.7) as a regularity condition is quite different from that used in [10]. The formalism considercd in the present article is designed for the analysis of massive whence singular data while in [10] only massless data have been considered.

In [10] has been exhibited a class of metrics which are analytic and satisfy condition (4.7) but which are not conformally flat near $i$. These metrics do not imply conformal structures on compact manifolds. However, Lemma 2.1 shows that conformal structures on compact manifolds which have positive Yamabe number and satisfy condition (4.7) at a given number of points are abundant among all conformal structures with positive Yamabe number.

To prove assertion (i) of Theorem 4.1 we discuss the normal expansion of the analytic lift of the spinor field

$$
\begin{aligned}
|x|^{3} d_{a b c d}^{W}= & -\frac{3}{2} \frac{1}{|x|^{2}} U W D_{(a b} \Gamma D_{c d)} \Gamma+U W D_{(a b} D_{c d)} \Gamma \\
& +2\left\{W D_{(a b} \Gamma D_{c d)} U-3 U D_{(a b} \Gamma D_{c d)} W\right\} \\
& +2 \Gamma\left\{-U D_{(a b} D_{c d)} W-W D_{(a b} D_{c d)} U\right. \\
& \left.\quad+6 D_{(a b} U D_{c d)} W+U W s_{a b c d}\right\} \\
& +|x|^{3}\left\{-2 W D_{(a b} D_{c d)} W+6 D_{(a b} W D_{c d)} W+W^{2} s_{a b c d}\right\}
\end{aligned}
$$

on $B_{a}(i) \backslash\{i\}$. 
Observing that $D_{a b} \Gamma=2 \rho x_{a b}$ on $C_{a}$, we find that the first term on the right-hand side lifts to

$$
-\frac{3}{2} \frac{1}{\rho^{2}} U W D_{(a b} \Gamma D_{c d)_{j}} \Gamma=-12 U W \epsilon_{(a b c d)_{j}}^{2}
$$

on $C_{a}$ where we make use of the constant coefficients

$$
\epsilon_{a b c d}^{k} \equiv \epsilon_{(a}^{(e} \epsilon_{b}^{f} \epsilon_{c}^{g} \epsilon_{d)}^{h)_{k}} .
$$

Since scalar functions have expansion type $p$, this term has expansion type $p$. It satisfies the symmetry condition because

$$
\epsilon_{(a b c d) j}^{2}=0 \text { for } j=0,4
$$

The second term has lift

$$
U W D_{(a b} D_{c d)_{j}} \Gamma=-4 U W \rho \check{\gamma}_{(a b}{ }^{e}{ }_{c} x_{d)_{j} e} .
$$

Since $s_{a b c d}$ and $r$ have expansion type $p+2, p$, respectively, it follows by induction from the structure equations (3.23) and (3.24) that the coefficients $\check{\gamma}_{a b c d}$ have expansion type $p+1$. Therefore, the function above has expansion type $p$. It holds

$$
\check{\gamma}_{(a b}{ }^{e}{ }_{c} x_{d)_{0} e}=-\frac{1}{\sqrt{2}} \check{\gamma}_{0000}, \quad \check{\gamma}_{(a b}{ }^{e}{ }_{c} x_{d)_{1} e}=\frac{1}{\sqrt{2}} \check{\gamma}_{1111} .
$$

Observing (3.13) and (3.17) we get

$$
D_{a b} D^{a b} \Gamma+6=2 \sqrt{2} \rho\left(\check{\gamma}_{1100}-\check{\gamma}_{0011}\right),
$$

which entails with (3.25) for the first coefficient function in (2.24)

$$
U_{0}=\exp \left\{\sqrt{2} \int_{0}^{\rho} \check{\gamma}_{1100} \mathrm{~d} s\right\}
$$

Eqs. (3.23) and (3.24) are ordinary differential equations for $\check{\gamma}_{0000}, \check{\gamma}_{1111}$ if the quantities $\check{\gamma}_{0011}, s_{0000}, s_{1111}$ are considered as given. Their solution can be represented in view of Eqs. (3.25) and (4.11) in the form

$$
\rho \check{\gamma}_{(a b}{ }^{e}{ }_{c} x_{d)_{j} e}=\frac{U_{0}^{2}}{2 \rho} \int_{0}^{\rho} \frac{\rho^{\prime 2} s_{(a b c d)_{j}}}{U_{0}^{2}} \mathrm{~d} \rho^{\prime} \quad \text { for } j=0,4 .
$$

Since $U_{0}^{-2} s_{(a b c d)}$ has an expansion of type $p+2$ and satisfies (3.41), the integral divided by $\rho$ has expansion type $p$ and satisfies the symmetry condition. Since because of Lemma 3.1 these properties are not affected hy multiplication with analytic functions, the term above has the desired properties. The third term lifts to 


$$
\begin{aligned}
& 2\left\{W D_{(a b} \Gamma D_{c d)_{j}} U-3 U D_{(a b} \Gamma D_{c d)_{j}} W\right\} \\
& =4 \rho\left\{W x_{(a b} D_{c d)_{j}} U-3 U x_{(a b} D_{c d)_{j}} W\right\} .
\end{aligned}
$$

This term is of type $p$, since the lift of a smooth 1 -form is of type $p+1$. It satisfies the symmetry condition because $x_{(a b) j}-0$ for $j-0,2$.

Being a product of a smooth tensor field of rank 2 with a factor $\rho^{2}$, the lift of the fourth term is of expansion type $p$ and it satisfies the symmetry condition because of (3.41). Because of the factor $\rho^{3}$, the last term in (4.10) is in fact of expansion type $p-1$ and thus of the desired type. This proves the assertion (i) of Theorem 4.1 because multiplication with the lift of $\kappa^{\prime}$ does not change the expansion type.

To prove the remaining assertions of the theorems above we shall analyse the behaviour of the analytic tensor field (4.9). The function $\Omega^{\prime}$ is the unique analytic function satisfying the conditions

$$
\Omega^{\prime}=0, \quad D_{j} \Omega^{\prime}=0, \quad D_{j} D_{k} \Omega^{\prime}=-2 h_{j k} \quad \text { at } i
$$

and the equation

$$
2 \Omega^{\prime} D_{j} D^{j} \Omega^{\prime}-3 D_{j} \Omega^{\prime} D^{j} \Omega^{\prime}+\frac{1}{2} r \Omega^{\prime 2}=0
$$

equivalent to the Yamabe equation. Instead of using the Hadamard procedure, the expansion of $\Omega^{\prime}$ can be determined directly by taking derivatives of (4.13) and evaluating at $i$. By this procedure we get

$$
\begin{aligned}
& D_{l} D_{k} D_{j} \Omega^{\prime}(i)=0 \\
& D_{m} D_{l} D_{k} D_{j} \Omega^{\prime}(i)=-\frac{4}{3} r_{m(j k) l}+4 h_{(m l} r_{k j)}-r h_{(m l} h_{k j)} . \\
& D_{p} D_{m} D_{l} D_{k} D_{j} \Omega^{\prime}(i)=-\left(D_{m} r_{p(j k) l}+D_{p} r_{l(j k) m}+D_{l} r_{m(j k) p}\right) \\
&+10 h_{(p m} D_{l} r_{k j)}-\frac{5}{2} h_{(p m} h_{l k} D_{j)} r .
\end{aligned}
$$

From this we obtain the following expansion coefficients at $i$ :

$$
\begin{aligned}
& e_{i j}^{\prime}=0, \quad D_{k} e_{i j}^{\prime}=0, \quad D_{l} D_{k} e_{i j}^{\prime}=0, \\
& D_{m} D_{l} D_{k} e^{\prime i j}=2 h_{(k}{ }^{(i} k_{l m)}{ }^{j)}+2 h_{(k l} k^{(i}{ }_{m)}^{j)} \text {. }
\end{aligned}
$$

We denote the spinor field corresponding to $e_{j k}^{\prime}$ and its analytic lift by $e_{a b c d}^{\prime}$. Using (4.15). (4.3), and the procedure discussed in Section 2 for calculating normal expansions, we find for the function $e_{j}^{\prime}=e_{(a b c d)_{j}}^{\prime}$ the expansion

$$
e_{j}^{\prime}=e_{j .3}^{\prime} \rho^{3}+\mathrm{O}\left(\rho^{4}\right)
$$

with

$$
e_{j .3}^{\prime}=(2-j) \frac{\sqrt{2}}{6} \sum_{k=0}^{4}\left(\begin{array}{l}
4 \\
k
\end{array}\right)^{1 / 2} b_{(a b c d)_{k}}^{*} T_{4}{ }^{k}{ }_{j} .
$$


Whereas we would expect by our considerations in Section 2 an expansion of the type

$$
e_{j, 3}^{\prime}=\sum_{q=|2-j|}^{5} \sum_{k=0}^{2 q} e_{j, 3 ; 2 q, k}^{\prime} T_{2 q}{ }^{k}{ }_{q-2+j}
$$

we see that, in agreement with Theorem 4.1 , the terms of order $q>p-1=2$ vanish. Furthermore, we observe that the coefficients $e_{j, 3 ; 4, k}^{\prime}$ do not vanish in general and that

$$
e_{4,3 ; 4, k}^{\prime}=-e_{0,3 ; 4, k}^{\prime}
$$

To determine derivatives of $e_{i j}^{\prime}$ of higher order is already very awkward and we shall use other methods to discuss the structure of the expansion coeffficients in a general way.

Lemma 4.3. The tensor field $e_{j k}^{\prime}$ satisfies the equations

$$
\begin{aligned}
& \Omega^{\prime} D^{i} e_{i j}^{\prime}-2 D^{i} \Omega^{\prime} e_{i j}^{\prime}=0, \\
& 2\left(D_{i} e_{j l}^{\prime}-D_{j} e_{i l}^{\prime}\right)-h_{i l} D^{k} e_{k j}^{\prime}+h_{j l} D^{k} e_{k i}^{\prime}+2 \Omega^{\prime} k_{l i j}=0
\end{aligned}
$$

on $B_{a}(i)$ which read in space-spinor notation

$$
\begin{aligned}
& \Omega^{\prime} D^{a b} e_{a b c d}^{\prime}-2 D^{a b} \Omega^{\prime} e_{a b c d}^{\prime}=0, \\
& D_{(a}^{f} e_{b c d) f}^{\prime}-\Omega^{\prime} b_{a b c d}=0 .
\end{aligned}
$$

Eq. (4.18) is only a rewriting of the constraint equations satisfied by $d_{j k}^{\prime}$ (cf. [12]) in terms of $e_{j k}^{\prime}$. The integrability condition (4.19) is obtained as follows. From (4.9) we get

$$
\begin{aligned}
D^{i} e_{i k}^{\prime}= & D^{i} D_{i} D_{k} \Omega^{\prime}-\frac{1}{3} D_{k}\left(D^{i} D_{i} \Omega^{\prime}+\frac{1}{4} \Omega^{\prime} r\right)+D^{i} \Omega^{\prime} l_{i k}+\Omega^{\prime} D^{i} l_{i k} \\
= & D_{k} D^{i} D_{i} \Omega^{\prime}+D^{i} \Omega^{\prime}\left(l_{i k}+\frac{1}{4} r h_{i k}\right)-\frac{1}{3} D_{k}\left(D^{i} D_{i} \Omega^{\prime}+\frac{1}{4} \Omega^{\prime} r\right) \\
& +D^{i} \Omega^{\prime} l_{i k}+\Omega^{\prime} D^{i} l_{i k} \\
= & 2\left(D^{i} \Omega^{\prime} l_{i k}+\frac{1}{3} D_{k}\left(D^{i} D_{i} \Omega^{\prime}+\frac{1}{4} \Omega^{\prime} r\right)\right) .
\end{aligned}
$$

Taking a derivative of (4.9) and antisymmetrizing we get

$$
\begin{aligned}
& D_{j} e_{i k}^{\prime}-D_{i} e_{j k}^{\prime} \\
& =-D^{h} \Omega^{\prime} r_{h k j i}+D_{j} \Omega^{\prime} l_{i k}-D_{i} \Omega^{\prime} l_{j k}+\Omega^{\prime} k_{k i j} \\
& \quad-\frac{1}{3} h_{i k} D_{j}\left(D_{l} D^{l} \Omega^{\prime}+\frac{1}{4} \Omega^{\prime} r\right)+\frac{1}{3} h_{j k} D_{i}\left(D_{l} D^{l} \Omega^{\prime}+\frac{1}{4} \Omega^{\prime} r\right)
\end{aligned}
$$

which, together with the preceding equation and (4.1) gives (4.19).

As noted above, the conditions of Theorems 4.1 and 4.2 are invariant under conformal rescalings. This allows us to employ a particular conformal gauge to simplify the arguments. To explain this gauge we consider certain conformal geodesics of $\left(B_{a}(i), h\right)$ through the point $i$. These are given by solutions $(x(t), b(t))$ of the conformal geodesic equations

$$
\begin{aligned}
& D_{\dot{x}} \dot{x}=-2\langle b, \dot{x}\rangle \dot{x}+h(\dot{x}, \dot{x}) h^{\#}(b, \cdot), \\
& D_{\dot{x}} b=\langle b, \dot{x}\rangle b-\frac{1}{2} h^{\#}(b, b) \dot{x}+l(\dot{x}, \cdot)
\end{aligned}
$$


with initial conditions

$$
x(0)=i, \quad h(\dot{x}, \dot{x})=-1, \quad h(0)=0 .
$$

Here $x(t)$ denotes a space-time curve through $i, b(t)$ a 1 -form along that curve, and by $h^{\#}$ we denote the contravariant form of $h$. Assuming that $a$ is small enough, there exists on $B_{a}(i)$ a unique conformal rescaling of the metric which is analytic, keeps the metric and the connection unchanged at $i$, and which is such that if the solutions to (4.22)-(4.24) is expressed in terms of the rescaled metric, we have

$$
\langle b, \dot{x}\rangle=0 \quad \text { on } B_{a}(i) .
$$

A metric in the conformal class for which condition (4.25) is satisfied along solutions to (4.22)-(4.24) will be said to satisfy the "cn-gauge". In the following we shall assume our metric to satisfy the cn-gauge.

Along any conformal geodesic the relations

$$
\begin{aligned}
& D_{\dot{x}}(h(\dot{x}, \dot{x}))=-2\langle b, \dot{x}\rangle h(\dot{x}, \dot{x}), \\
& D_{\dot{x}}\langle b, \dot{x}\rangle=-\langle b, \dot{x}\rangle^{2}+\frac{1}{2} h^{\#}(b, b) h(\dot{x}, \dot{x})+l(\dot{x}, \dot{x})
\end{aligned}
$$

are satisfied. Therefore, we get in cn-gauge along the conformal geodesics satisfying (4.22)(4.24) the relations

$$
\begin{aligned}
& D_{\dot{x}}(h(\dot{x}, \dot{x}))=0, \\
& l(\dot{x}, \dot{x})=-\frac{1}{2} h^{\#}(b, b) h(\dot{x}, \dot{x}) .
\end{aligned}
$$

It will be convenient to consider the complex analytic extension of the space $\left(B_{a}(i), h\right)$ near $i$. We assume that there exists a three-dimensional complex analytic metric manifold $(B, h)$ (i.e. $h$ defines a complex-valued non-degenerate scalar product) which contains $\left(B_{a}(i), h\right)$ as a real Riemannian subspace and which is such that $B$ is a convex normal neighbourhood with respect to $h$ and that the function $\Gamma$ has an analytic extension to $B$. The exact size of $B$ is not important to us; it can easily be seen that $B$ can always be chosen such that the statements made in the following are true. The restriction of $S U(S)$ to $B_{a}(i)$ has a complex analytic extension to a bundle $S L(B)$ of spin frames on $B$ with structure group $S L(2, C)$. We denote the analytic extensions of the connection and the solder form again by $\check{\omega}_{b}^{a}, \sigma^{a b}$, respectively and note that the first and second structure equation hold unchanged on the complex analytic extension. The standard horizontal vector fields $H_{c} d$ are defined on $S L(B)$ by the requirements

$$
\left\langle\check{\omega}_{b}^{a}, H_{c d}\right\rangle=0, \quad\left\langle\sigma^{a b}, H_{c d}\right\rangle=h_{c d}{ }^{a b} .
$$

The complex cone which is generated by the null geodesics through $i$ will be denoted by $N$. We assume that $\Omega^{\prime}$ has a complex analytic extension with $U \neq 0$ on $B$. The field $e_{j k}^{\prime}$ is then also extended by (4.9). We have $N=\{\Gamma=0\}=\left\{\Omega^{\prime}=0\right\}, \mathrm{d} \Gamma, \mathrm{d} \Omega \neq 0$ on $N \backslash\{i\}$ and $D^{k} \Gamma, D^{k} \Omega^{\prime}$ are tangent to the null generators of $N$ by (2.22) and (4.13).

Since we assumed the cn-gauge before extending into the complex domain, conditions (4.26) and (4.27) will remain true along conformal geodesics through $i$ in $B$. The first 
condition implies that null generators of $N$ are conformal geodesics (considered as point sets) and the second condition then implies that

$$
D^{k} \Gamma D^{j} \Gamma s_{j k}=D^{k} \Gamma D^{j} \Gamma l_{j k}=0 \quad \text { on } N .
$$

To exploit the fact that the field $e_{j k}^{\prime}$ is defined in terms of the unique analytic solution $\Omega^{\prime}$ of (4.12) and (4.13), it is convenient to introduce a certain submanifold $\check{B}$ of $S L(B)$ which is constructed as follows. We consider the group

$$
G=\left\{s(z) \in S L(2, C) \mid s(z)=\left(\begin{array}{ll}
1 & 0 \\
z & 1
\end{array}\right), z \in C\right\} .
$$

Suppose $\delta^{*}$ is the spin frame at $i$ on which was based the construction of $C_{a}$. Let $\gamma$ be the generator of $N$ which is tangent to $\delta_{1}^{*} \delta_{1}^{*}$. Denote by $\delta(z)$ the spin frame which is obtained by the action of $s(z) \in G$ on $\delta^{*}$ such that $\delta(0)=\delta^{*}$, denote by $y \rightarrow \delta(y, z)$ the integral curve of $H_{11}$ satisfying $\delta(0, z)=\delta(z)$, by $x \rightarrow \delta(x, y, z)$ the integral curve of $H_{00}$ satisfying $\delta(0, y, z)=\delta(y, z)$, and finally by $D$ the set of $(x, y, z) \in C^{3}$ for which the latter are defined. The set $\breve{B}=\{\delta(x, y, z) \mid(x, y, z) \in D\}$ sweeps out a complex analytic threedimensional submanifold of $S L(S)$ with global coordinates $z^{1}=x, z^{2}=y, z^{3}=z$. We denote by $\check{\pi}$ the restriction of the projection $\pi$ to $\check{B}$ and define the subsets $\breve{i}=\{\delta(0,0, z) \mid z \in$ $C\}, \check{N}=\{\delta(x, 0, z) \mid(x, 0, z) \in D, x \neq 0\}, \check{\gamma}=\{\delta(0, y, z) \mid(0, y, z) \in D\}$ of $\check{B}$ which project onto $i, N \backslash \gamma, \gamma$, respectively.

A spinor field $\xi$ on $B$ is represented on $\breve{B}$ by an invariant spinor-valued function to which we refer as to the lift of $\xi$. In the following there will be made no distinction in the notation for the fields on $B$ and their lifts to $\check{B}$. It will be clear from the context what is meant. The trace-free part of the Ricci tensor is then represented on $\check{B}$ by the function $s_{a b c d}$.

The map $\check{\pi}$ has rank equal to 2 at the points of $\check{\gamma}$. Since its rank is 3 on $\check{B} \backslash \check{\gamma}$, the condition

$$
\left\langle\sigma^{c d}, c_{a b}\right\rangle=h_{a b}{ }^{c d}
$$

defines there analytic vector fields $c_{a b}$. By arguments similar to those given in [9] we see that

$$
c_{a b}=c_{a b}^{\alpha} \frac{\partial}{\partial z^{\alpha}}
$$

with coefficients such that

$$
c^{\alpha}{ }_{00}=\delta_{1}^{\alpha} \quad \text { on } \check{B}, \quad c^{3} 01=\frac{1}{2 x}+\check{c}^{3} 01 \quad \text { on } \check{B} \backslash \check{\gamma}
$$

and $c^{\alpha}{ }_{11}, c^{1}{ }_{01}, c^{2}{ }_{01}, \breve{c}^{3} 01$ are analytic on $\check{B}$ with

$$
c_{11}^{1}=c_{11}^{3}=c_{01}^{1}=c_{01}^{2}=\check{c}_{01}^{3}=0, \quad c_{11}^{2}=1 \quad \text { on } \check{\gamma} .
$$

The connection coefficients

$$
\gamma_{c d}{ }_{b}{ }_{b} \equiv\left\langle\check{\omega}_{b}^{a}, c_{c d}\right\rangle=\frac{1}{x} \gamma_{c d}^{\prime}{ }^{a}{ }_{b}+\check{\gamma}_{c d}{ }^{a}{ }_{b}
$$


satisfy

$$
\gamma_{c d u b}^{\prime}=-\epsilon_{(c}{ }^{0} \epsilon_{d)}{ }^{1} \epsilon_{a}{ }^{0} \epsilon_{b}{ }^{0}, \quad \gamma_{00 a b}=0, \quad \check{\gamma}_{c d a b}=\mathbf{O}(|x|) \quad \text { as } x \rightarrow 0 .
$$

The functions $\check{\gamma}_{c d a b}$ extend by analyticity to $\check{B}$. Again, spinor fields on $B$ are represented by invariant spinor-valued functions on $\check{B}$.

Since $D_{a b} \Omega^{\prime}=D_{11} \Omega^{\prime} \epsilon_{a}{ }^{1} \epsilon_{a}{ }^{1}$ on $\check{N}$, condition (4.29) translates into

$$
s_{0000}=0 \text { on } \check{N} \text {. }
$$

For given $p=0,1,2, \ldots$ this implies $D_{00}^{p} s_{0000}=0$ on $\check{N}$. Taking the limit to $i$ along a fixed null generator of $\check{N}$, we find that this implies at $i$ in terms of our earlier notation

$$
s^{a_{p}} s_{0}^{b_{p}}{ }_{0} \cdots s^{a_{1}}{ }_{0} s^{b_{1}}{ }_{0}{ }^{a}{ }_{0} s^{b}{ }_{0} s^{b}{ }_{0} s_{0}{ }_{0} D_{a_{p} b_{p}} \cdots D_{a_{1} b_{1}} s_{a b c d}^{*}=0
$$

for $s^{a}{ }_{0}=s^{a}{ }_{0}(z)$ with arbitrary $z$. This entails

$$
D_{\left(a_{p} b_{p}\right.} \cdots D_{a_{1} b_{1}} s_{a b c d)}^{*}=0, \quad p=0,1,2, \ldots,
$$

which in turn implies (4.30).

The Cotton tensor is represented by the function $b_{a h r d}$ and, by the argument above, condition 4.7 translates into the equivalent condition

$$
b_{0000}=0 \quad \text { on } \check{N} \text {. }
$$

which proves the equivalence of conditions (i) and (ii) of Theorem 4.2.

Evalutating the first structure equation on $c_{00} \wedge c_{a b}$ yields the equations

$$
\begin{aligned}
& \partial_{x} c_{01}^{1}=-\frac{1}{x} c^{1} 01+2 \check{\gamma}_{0100} c_{01}^{1}-2 \check{\gamma}_{0101}, \\
& \partial_{x} c^{2}{ }_{01}=-\frac{1}{x} c^{2} 01+2 \check{\gamma}_{0100} c^{2} 01, \\
& \partial_{x} \breve{c}_{01}^{3}=-\frac{1}{x} \check{c}_{01}^{3}+2 \check{\gamma}_{0100} \check{c}^{3}{ }_{01}+\frac{1}{x} \check{\gamma}_{0100} .
\end{aligned}
$$

The second equation implies

$$
c^{2} 01=0
$$

whence

$$
c_{01}=c_{01}^{1} \partial_{x}+\left(\frac{1}{2 x}+\check{c}_{01}^{3}\right) \partial_{z}
$$

with

$$
c_{01}^{1}=-\frac{2}{x} p \int_{0}^{x} \frac{x^{\prime}}{p} \check{\gamma}_{0101} \mathrm{~d} x^{\prime}, \quad \check{c}^{3} 01=\frac{1}{2 x}(p-1), \quad p=\mathrm{e}^{2 \int_{0}^{x} \check{\gamma}_{0100} \mathrm{~d} x^{\prime}} .
$$

We get now furthermore the equations

$$
\partial_{x} c_{11}^{1}=2 \check{\gamma}_{1100} c_{01}^{1}-\check{\gamma}_{1101}, \quad \partial_{x} c_{11}^{2}=0, \quad \partial_{x} c_{11}^{3}=\check{\gamma}_{1100}\left(\frac{1}{x}+2 \check{c}^{3} 01\right)
$$


which imply

$$
c_{11}=c^{1}{ }_{11} \partial_{x}+\partial_{y}+c^{3}{ }_{11} \partial_{z}
$$

with coefficients which are given by simple integrals. Evaluating the second structure equation on $c_{00} \wedge c_{a b}$ gives

$$
\begin{aligned}
& \partial_{x} \check{\gamma}_{0100}+\frac{2}{x} \check{\gamma}_{0100}-2 \check{\gamma}_{0100}^{2}=\frac{1}{2} s_{0000}, \\
& \partial_{x} \check{\gamma}_{0101}+\left(\frac{1}{x}-2 \check{\gamma}_{0100}\right) \check{\gamma}_{0101}=\frac{1}{2} s_{0001}, \\
& \partial_{x} \check{\gamma}_{0111}+\left(\frac{1}{x}-2 \check{\gamma}_{0100}\right) \check{\gamma}_{0111}=\frac{1}{2} s_{0011}-\frac{r}{12}, \\
& \partial_{x} \check{\gamma}_{1100}+\frac{1}{x} \check{\gamma}_{1100}-2 \check{\gamma}_{0100} \check{\gamma}_{1100}=s_{0001}, \\
& \partial_{x} \check{\gamma}_{1101}-2 \check{\gamma}_{0101} \check{\gamma}_{1100}=s_{0011}+\frac{1}{12} r, \\
& \partial_{x} \check{\gamma}_{1111}-2 \check{\gamma}_{0111} \check{\gamma}_{1100}=s_{0111} .
\end{aligned}
$$

Observing (4.30), the first of these equations, if expressed in terms of the unknown $u=$ $x^{2} \check{\gamma}_{0100}$, takes on $\check{N}$ the form

$$
\dot{u}=\frac{2}{x^{2}} u^{2} .
$$

The general solution of this equation is given by

$$
u=\frac{a x}{2 a+c x}, \quad a, c=\text { const. }, \quad|a|+|c| \neq 0 .
$$

Since $u=\mathrm{O}\left(|x|^{2}\right)$ as $x \rightarrow 0$, it follows that $u$ whence $\check{\gamma}_{0100}$ vanishes on $\check{N}$.

Integration of the remaining structure equations gives on $\check{N}$

$$
\begin{aligned}
& \gamma_{0100}=-\frac{1}{2 x}, \quad \gamma_{0101}=\frac{1}{2 x} \int_{0}^{x} x^{\prime} s_{0001} \mathrm{~d} x^{\prime}, \\
& \gamma_{0111}=\frac{1}{2 x} \int_{0}^{x} x^{\prime}\left(s_{0011}-\frac{r}{6}\right) \mathrm{d} x^{\prime}, \quad \gamma_{1100}=\frac{1}{x} \int_{0}^{x} x^{\prime} s_{0001} \mathrm{~d} x^{\prime}, \\
& \gamma_{1101}=\int_{0}^{x}\left(2 \gamma_{0101} \gamma_{1100}+s_{0011}+\frac{r}{12}\right) \mathrm{d} x^{\prime}, \quad \gamma_{1111}=\int_{0}^{x}\left(2 \gamma_{0111} \gamma_{1100}+s_{0111}\right) \mathrm{d} x^{\prime}, \\
& c_{01}=c^{1}{ }_{01} \partial_{x}+\frac{1}{2 x} \partial_{z}, \quad c_{11}=c^{1}{ }_{11} \partial_{x}+\partial_{y}+c^{3}{ }_{11} \partial_{z}, \\
& c^{1} 01=-\frac{2}{x} \int_{0}^{x} x^{\prime} \gamma_{0101} \mathrm{~d} x^{\prime}, \quad c^{\prime}{ }_{11}=\int_{0}^{x}\left(2 \gamma_{1100} c^{1}{ }_{01}-2 \gamma_{1101}\right) \mathrm{d} x^{\prime},
\end{aligned}
$$




$$
c^{3} 11=\int_{0}^{x} \frac{1}{x^{\prime}} \gamma_{1100} \mathrm{~d} x^{\prime} .
$$

Lemma 4.4. On $\breve{N}$ holds $s_{0001}=-\frac{1}{x^{2}} \int_{0}^{x} x^{\prime 2} b_{0000} \mathrm{~d} x^{\prime}$.

From (4.4) and the coefficients determined above we get on $\check{N}$

$$
\begin{aligned}
-b_{0000} & \equiv D_{00} s_{0001}-D_{01} s_{0000}=\partial_{x} s_{0001}-c_{01}\left(s_{0000}\right)+4 \gamma_{01}{ }^{f} s_{000 f} \\
& =\partial_{x} s_{0001}+\frac{2}{x} s_{0001}=\frac{1}{x^{2}} \partial_{x}\left(x^{2} s_{0001}\right)
\end{aligned}
$$

whence the result.

We shall now analyse the behaviour of the function $\Omega^{\prime}$ near $\check{N}$. Taking a derivative of (4.13) and restricting to $N$ gives there

$$
0=3 D^{i} \Omega^{\prime} D_{i} D_{j} \Omega^{\prime}-D_{j} \Omega^{\prime} \Delta \Omega^{\prime}
$$

Lifting these equations to $\check{N}$ and using the metric and connection coefficients calculated above we find as the only non-trivial equation in this system

$$
\begin{aligned}
0 & =3 D_{00} D_{11} \Omega^{\prime}-2\left(D_{00} D_{11} \Omega^{\prime}-D_{01} D_{01} \Omega^{\prime}\right) \\
& =\partial_{x} D_{11} \Omega^{\prime}-\frac{1}{x} D_{11} \Omega^{\prime}=x \partial_{x}\left(\frac{1}{x} D_{11} \Omega^{\prime}\right) .
\end{aligned}
$$

Since $\lim _{x \rightarrow 0}\left(\frac{1}{x} D_{11}\right)=D_{00} D_{11} \Omega^{\prime}(i)=-2$ this equation implies

$$
D_{11} \Omega^{\prime}=-2 x \text { on } \breve{N} \text {. }
$$

From this we get by direct calculation on $\breve{N}$

$$
\begin{aligned}
& D_{00} D_{00} \Omega^{\prime}=0, \quad D_{00} D_{01} \Omega^{\prime}=0, \\
& D_{00} D_{11} \Omega^{\prime}=2, \quad D_{01} D_{01} \Omega^{\prime}=1, \\
& D_{01} D_{11} \Omega^{\prime}=-4 x \partial_{x}\left(\frac{u}{x}\right), \\
& u=\frac{1}{2} \int_{0}^{x}\left(\int_{0}^{x^{\prime}} x^{\prime \prime} s_{0001} \mathrm{~d} x^{\prime \prime}\right) \mathrm{d} x^{\prime}, \quad \Delta_{h} \Omega^{\prime}=-6 .
\end{aligned}
$$

To determine the value of $D_{11} D_{11} \Omega^{\prime}$ on $\check{N}$ we differentiate (4.13) twice. interchange derivatives, and restrict to $\check{N}$ to obtain

$$
\begin{aligned}
0= & 6 D^{i} \Omega^{\prime} D_{i} D_{k} D_{j} \Omega^{\prime}-2 D_{j} \Omega^{\prime} \Delta D_{k} \Omega^{\prime}-2 D_{k} \Omega^{\prime} \Delta D_{j} \Omega^{\prime} \\
& +6 D_{k} D^{i} \Omega^{\prime} D_{i} D_{j} \Omega^{\prime}-2 \Delta \Omega^{\prime} D_{k} D_{j} \Omega^{\prime}-\frac{2}{3} r D_{k} \Omega^{\prime} D_{j} \Omega^{\prime} \\
& -4 D_{k} \Omega^{\prime} s_{j i} D^{i} \Omega^{\prime}-4 D_{j} \Omega^{\prime} s_{k i} D^{i} \Omega^{\prime}+6 h_{k j} s_{i l} D^{i} \Omega^{\prime} D^{l} \Omega^{\prime} .
\end{aligned}
$$

In view of the quantities determined above we derive from this the equation 


$$
\begin{aligned}
x^{2} \partial_{x}\left(\frac{1}{x} D_{11} D_{11} \Omega^{\prime}\right)=f(x) \equiv & 4 x\left(c_{01}^{1} \partial_{x}+\frac{1}{2 x} \partial_{z}\right) D_{01} D_{11} \Omega^{\prime} \\
& +8 x \gamma_{0101} D_{01} D_{11} \Omega^{\prime}+12\left(D_{01} D_{11} \Omega^{\prime}\right)^{2} .
\end{aligned}
$$

Since the expansion coefficients (4.14) give

$$
\lim _{x \rightarrow 0}\left(\frac{1}{x} D_{11} D_{11} \Omega^{\prime}\right)=D_{00} D_{11} D_{11} \Omega^{\prime}(i)=0,
$$

this entails

$$
D_{11} D_{11} \Omega^{\prime}=x \int_{0}^{x} \frac{f\left(x^{\prime}\right)}{x^{\prime 2}} \mathrm{~d} x^{\prime} .
$$

Using (4.33), (4.34), and Lemma 4.4 we find on $\check{N}$

$$
e_{00 c d}^{\prime}=0
$$

a relation which can also be obtained by restricting Eq. (4.20) to $\check{N}$. It does not require any assumption on the gauge or on the conformal structure. Furthermore

$$
\begin{aligned}
e_{0111}^{\prime}= & \int_{0}^{x}\left(\frac{2}{x^{\prime 2}} \int_{0}^{x^{\prime}} x^{\prime \prime} b_{0000} \mathrm{~d} x^{\prime \prime}\right) \mathrm{d} x^{\prime}, \\
e_{1111}^{\prime}= & x \int_{0}^{x} \frac{1}{x^{\prime 2}}\left\{4 x^{\prime}\left(c^{1} 01 \partial_{x^{\prime}}+\frac{1}{2 x^{\prime}} \partial_{z}\right) e_{0111}^{\prime}\right. \\
& \left.+8 x^{\prime} \gamma_{0101} e_{0111}^{\prime}+12\left(e_{0111}^{\prime}\right)^{2}\right\} \mathrm{d} x^{\prime} .
\end{aligned}
$$

It follows that $e_{a b c d}^{\prime}$ vanishes on $\check{N}$ if and only if $b_{0000}$ vanishes there. Since $\Omega^{\prime-1} e_{a b c d}^{\prime}$ extends to an analytic tensor field on $B_{a}(i)$ if and only if $e_{a b c d}^{\prime}$ vanishes on $\check{N}$ (cf. [17]), this shows the equivalence of conditions (ii) and (iii) of Theorem 4.2.

Condition (iv) of Theorem 4.2 is equivalent to the vanishing of $D_{e f} e_{a b c d}^{\prime}$ on $\check{N}$ (cf. [10]). Since $e_{a b c d}^{\prime}=0$ at $x=0$ by (4.15), it follows immediately that condition (iv) implies (iii). To show the reverse we assume now that $e_{a b c d}^{\prime}=0$ whence $b_{0000}=0$ and $s_{0001}=0$ on $\check{N}$. This leads to some simplifications in the values of the metric and connection coefficients given above.

Since $D_{00}, D_{01}$ are interior operators on $\check{N}$ we have

$$
D_{0 f} e_{a b c d}^{\prime}-0 \text { on } \check{N} \text {. }
$$

In view of these equations the restriction of the integrability condition (4.21) to $\check{N}$ gives

$$
0=D_{(a}^{e} e_{b c d) e}^{\prime}=D_{1(a} e_{b c d) 0}^{\prime}
$$


whence

$$
0=D_{e f} e_{a b c 0}^{\prime} \text { on } \check{N}
$$

It remains to determine on $\breve{N}$ the value of

$$
D_{11} e_{1111}^{\prime}=D_{11} D_{11} D_{11} \Omega^{\prime}+D_{11} \Omega^{\prime} s_{1111} \text {. }
$$

By taking three covariant derivatives of Eq. (4.13). interchanging the order of derivatives, and restricting to $N$, we get

$$
\begin{aligned}
0= & 6 D^{i} \Omega^{\prime} D_{i} D_{l} D_{k} D_{j} \Omega^{\prime}-6 D^{i} \Omega^{\prime} D_{l} r_{h j k i} D^{h} \Omega^{\prime} \\
& -6 D^{i} \Omega^{\prime}\left(r_{h k l i} D^{h} D_{j} \Omega^{\prime}+r_{h j l i} D^{h} D_{k} \Omega^{\prime}\right. \\
& \left.\quad+r_{h k j i} D^{h} D_{l} \Omega^{\prime}+r_{h j k i} D^{h} D_{l} \Omega^{\prime}\right) \\
& +6 D_{l} D_{k} D_{i} \Omega^{\prime} D^{i} D_{j} \Omega^{\prime}+6 D_{l} D_{i} D_{j} \Omega^{\prime} D^{i} D_{k} \Omega^{\prime} \\
& +6 D_{i} D_{k} D_{j} \Omega^{\prime} D^{i} D_{l} \Omega^{\prime}-2 D_{l} D_{k} D_{j} \Omega^{\prime} \Delta \Omega^{\prime} \\
& -2 D_{l} D_{k} \Omega^{\prime} D_{j} \Delta \Omega^{\prime}-2 D_{l} D_{j} \Omega^{\prime} D_{k} \Delta \Omega^{\prime}-2 D_{k} D_{j} \Omega^{\prime} D_{l} \Delta \Omega^{\prime} \\
& -r D_{j} \Omega^{\prime} D_{l} D_{k} \Omega^{\prime}-r D_{k} \Omega^{\prime} D_{l} D_{j} \Omega^{\prime}-r D_{l} \Omega^{\prime} D_{k} D_{j} \Omega^{\prime} \\
& -2 D_{j} \Omega^{\prime} D_{l} D_{k} \Delta \Omega^{\prime}-2 D_{k} \Omega^{\prime} D_{l} D_{j} \Delta \Omega^{\prime}-2 D_{l} \Omega^{\prime} D_{k} D_{j} \Delta \Omega^{\prime} \\
& -D_{j} r D_{l} \Omega^{\prime} D_{k} \Omega^{\prime}-D_{k} r D_{l} \Omega^{\prime} D_{j} \Omega^{\prime}-D_{l} r D_{k} \Omega^{\prime} D_{j} \Omega^{\prime} .
\end{aligned}
$$

In view of the quantities determined above this induces on $\check{N}$ as the only non-trivial equation

$$
\begin{aligned}
0= & 6 D_{11} \Omega^{\prime} \partial_{x} D_{11} D_{11} D_{11} \Omega^{\prime}-6\left(D_{11} \Omega^{\prime}\right)^{2} D_{11} r_{00111100} \\
& -24 D_{11} D_{11} D_{11} \Omega^{\prime}-3 D_{11} r\left(D_{11} \Omega^{\prime}\right)^{2}-6 D_{11} \Omega^{\prime} D_{11} D_{11} \Delta_{h} \Omega^{\prime} .
\end{aligned}
$$

Commuting the Laplacian in the last term with the covariant derivatives, expanding the Laplacian, and evaluating on $\check{N}$ gives

$$
\begin{aligned}
D_{11} D_{11} \Delta_{h} \Omega^{\prime}= & 2 \partial_{x} D_{11} D_{11} D_{11} \Omega^{\prime}+\frac{1}{x} D_{11} D_{11} D_{11} \Omega^{\prime} \\
& +D_{11} \Omega^{\prime}\left(D_{00} s_{1111}-2 D_{11} r_{1100}\right)
\end{aligned}
$$

Inserting this into the equation above we get

$$
\begin{aligned}
0 & =12 x \partial_{x} D_{11} D_{11} D_{11} \Omega^{\prime}-12 D_{11} D_{11} D_{11} \Omega^{\prime}-24 x^{2} D_{00} s_{1111} \\
& =12 x^{2} \partial_{x}\left\{\frac{1}{x}\left(D_{11} D_{11} D_{11} \Omega^{\prime}+D_{11} \Omega^{\prime} s_{1111}\right)\right\}
\end{aligned}
$$

Since by (4.14)

$$
\begin{aligned}
& \lim _{x \rightarrow 0}\left\{\frac{1}{x}\left(\begin{array}{lll}
D_{11} & D_{11} & \left.D_{11} \Omega^{\prime}+D_{11} \Omega^{\prime} s_{1111}\right)
\end{array}\right\}\right. \\
& =D_{00} D_{11} D_{11} D_{11} \Omega^{\prime}(i)-2 s_{1111}(i)=0,
\end{aligned}
$$

we conclude that $D_{11} e_{1111}^{\prime}=0$ on $\check{N}$. Thus we have shown the equivalence of conditions (iii) and (iv) of Theorem 4.2 . 
To prove assertions (ii) and (iii) of Theorem 4.1 we need to analyse the field $e_{j k}^{\prime}$ in more detail. Its expansion type is defined in terms of expansion (3.30). As indicated in (3.33) and (3.34), we write the symmetrized derivatives occurring in (3.32) in terms of completely symmetric spinors

$$
\begin{aligned}
D_{\left(b_{p} c_{p}\right.} \cdots D_{\left.b_{1} c_{1}\right)} \mathrm{e}^{\prime * a b c d}= & \mathrm{e}_{p, 0 ; b_{p} c_{p} \cdots b_{1} c_{1}}^{\prime 0}{ }^{a b c d} \\
& +\mathrm{e}_{p, 0 ;\left(b_{p} c_{p} \cdots b_{1}\right.}^{\prime 1}{ }^{(a b c} \epsilon_{\left.c_{1}\right)}{ }^{d)}+\cdots
\end{aligned}
$$

Eq. (3.31) shows that the contributions of type $p+2, p+1$ arise solely from the first two terms on the right-hand side of (4.37), while the third and fourth term contain a contribution of type $p, p-1$, respectively. The remaining contributions of type $p$ and $p-1$ are contained in first and second term on the right-hand side of

$$
\begin{aligned}
\mathrm{e}_{p, 1: b_{p} c_{p} \cdots b_{3} c_{3}}^{a^{*}}{ }^{a b c d} & =\frac{1}{2 p-1} \sum_{1 \leq k<h \leq p} D_{\left(b_{p} c_{p}\right.} \cdots D_{|e f|} \cdots D^{e f} \cdots D_{\left.b_{3} c_{3}\right)} \mathrm{e}^{i a b c d} \\
& =\mathrm{e}_{p, 1 ; b_{p} c_{p} \cdots b_{3} c_{3}}^{\prime 0}{ }^{a b c d}+\mathrm{e}_{p, 1 ;\left(b_{p} c_{p} \cdots b_{3}\right.}^{\prime 1}{ }^{(a b c} \epsilon_{\left.c_{3}\right)}{ }^{d)}+\cdots
\end{aligned}
$$

Here we use again the notation of (3.31)-(3.34).

The complex analytic extension of $e_{j k}^{\prime}$ is obtained by expanding it in the form (3.30), by setting $V^{a b}=|x| v^{a b}$ in this formula, and by allowing $V^{a b}$ to take all complex values. On $\check{N}$ we have $V^{a b}=x s^{a}{ }_{0} s^{b}{ }_{0}$ where $s^{a}{ }_{0}=s^{a}{ }_{0}(z)$ and $x, z$ are the coordinates introduced on $\breve{N}$ above. It follows from Eq. (3.31) that the restriction of $e_{a b c d}^{\prime}$ to $\check{N}$ contains exactly the information of (4.37). It can be extracted by observing the argument which led to the interpretation of (4.30) by (4.31). Observing (4.35), taking the limit of $D_{00}^{p} e_{00 c d}^{\prime}$ to $i$ along a fixed generator of $\check{N}$, and evaluating for all $z$ we obtain

$$
\mathrm{e}_{p, 0 ; b_{p} c_{p} \cdots b_{1} c_{1} a b c d}^{\prime 0}=0, \quad \mathrm{e}_{p, 0 ; b_{p} c_{p} \cdots b_{1} a b c}^{\prime 1}=0, \quad \mathrm{e}_{p, 0 ; b_{p} c_{p} \cdots c_{2} a b}^{\prime 2}=0, \quad p \geq 3 .
$$

Observing (4.36) and taking the limit of $D_{00}^{p} e_{0111}^{\prime}$ to $i$ we obtain

$$
\mathrm{e}_{p, 0 ; b_{p} c_{p} \cdots b_{4} c_{4} a b c d}^{\prime 3}=-\frac{8(p-1)(p-2)}{p+1} D_{\left(b_{p} c_{p}\right.} \cdots D_{b_{4} c_{4}} b_{a b c d)}^{*} .
$$

To derive the contributions of type $p$ and $p-1$ contained in (4.38), we need some information on the field $\Delta e_{a b c d}^{\prime} \equiv D_{g h} D^{g h} e_{a b c d}^{\prime}$ on $\check{N}$.

Lemma 4.5. Let $i, j, k$ be non-negative integers. The following equations hold on $\check{N}$ :

(i) $\Delta e_{000 a}^{\prime}=\epsilon_{a}^{1}\left\{2 \partial_{x}^{2} \mathrm{e}_{0111}+\frac{1}{x} \partial_{x} \mathrm{e}_{0111}-\frac{3}{x^{2}} \mathrm{e}_{0111}\right\}$,

(ii) $D_{0 f} \Delta e_{000}^{\prime} f=2 \partial_{x}^{3} e_{0111}^{\prime}+\frac{5}{x} \partial_{x}^{2} e_{0111}^{\prime}-\frac{2}{x^{2}} \partial_{x} e_{0111}^{\prime}$,

(iii) $D_{00}^{i} D_{g h} D_{00}^{j} D^{g h} D_{00}^{k} e_{000 a}^{\prime}=D_{00}^{i+j+k} \Delta e_{000 a}^{\prime}$,

(iv) if $i+j+k \geq 1$ and the operator $D^{i} D^{j} D^{k}$ of order $i+j+k$ is generated by $i+j+k-1$ factors $D_{00}$ and one factor $D_{01}$, then

$$
D^{i} D_{g h} D^{j} D^{g h} D^{k} e_{0000}^{\prime}=D_{00}^{i+j+k-1} D_{01} \Delta e_{0000}^{\prime} \text {. }
$$


The following relations hold on $\check{N}$. Eqs. (4.21) and (4.35) imply $D_{11} e_{000 a}^{\prime}=\epsilon_{a}{ }^{1} \partial_{x} e_{0111}^{\prime}$. From this and (4.35) we get by direct calculation

$D_{01} D_{01} e_{000 a}^{\prime}=\epsilon_{a}{ }^{1} \gamma_{0100}\left(D_{00} e_{0111}^{\prime}+6 \gamma_{0100} e_{0111}\right)$.

Since $D_{00} D_{11} e_{000 a}^{\prime}=D_{11} D_{00} e_{000 a}^{\prime}$ by (4.35), we get for

$$
\Delta e_{000 a}^{\prime}=\left(D_{00} D_{11}+D_{11} D_{00}-2 D_{01} D_{01}\right) e_{000 a}^{\prime}
$$

expression (i). From this (ii) is derived by direct calculation.

Relations (iii) and (iv) are obtained by repeated commutations of covariant derivatives. We shall only indicate a few steps.

We have $\left[D_{01}, D_{00}\right] D_{00}^{j} e_{a 000}^{\prime}=0$ since the commutator is given by terms of the form $r^{f}{ }_{a 0100} D_{00}^{j} e_{f 000}^{\prime}$ and $r^{f}{ }_{00100} D_{00}^{j} e_{a f 00}^{\prime}$, which vanish because of (4.35), and if $j \geq 1$ it contains terms of the form $r^{f}{ }_{00100} D_{00}^{i} D_{0 f} D_{00}^{k} e_{a 000}^{\prime}, i+k+1=j$, which vanish either because of (4.35) or because $2 r^{1} 00100=-s_{0000}=0$ in the cn-gauge. Since $D_{01} e_{a 000}^{\prime}=0$ by $(4.35)$, it follows inductively that

$$
D_{00}^{k} D_{01} D_{00}^{j} e_{a 000}^{\prime}=0, \quad k, j=0,1,2, \ldots
$$

We have $\left[D_{11}, D_{00}\right] D_{00}^{j} e_{a 000}^{\prime}=0$ by the result above and the arguments used to obtain it. It follows that

$$
D_{00}^{k} D_{01} D_{00}^{j+1} e_{a 000}^{\prime}=D_{00}^{k+1} D_{01} D_{00}^{j} e_{a 000}^{\prime}, \quad k, j=0,1,2, \ldots
$$

By these results and the arguments above we also get

$$
\begin{aligned}
& D_{01} D_{00}^{k+1} D_{01} D_{00}^{j} e_{a 000}^{\prime}=D_{00} D_{01} D_{00}^{k} D_{01} D_{00}^{j} e_{a 000}^{\prime}, \\
& D_{01} D_{00}^{k} D_{01} D_{00}^{j+1} e_{a 000}^{\prime}=D_{01} D_{00}^{k+1} D_{01} D_{00}^{j} e_{a 000}^{\prime}, \quad k, j=0,1,2, \ldots
\end{aligned}
$$

Using these relations we get (iii). The arguments leading to (iv) are lengthier, but the calculations are straightforward, quite similar to the ones indicated above, and will therefore not be given.

We shall now determine the remaining contributions of type $p$ and $p-1$. Relations (i) and (iii) of Lemma 4.5 imply on $\breve{N}$

$$
D_{00}^{i} D_{e f} D_{00}^{j} D^{e f} D_{00}^{k} e_{0000}^{\prime}=0 .
$$

Taking limits to $i$ along a fixed generators of $\breve{N}$ and evaluating for all $z$ we get from this

$$
D_{\left(a_{p} h_{p}\right.} \cdots D_{|e f|} \cdots D^{e f} \cdots D_{a_{3} b_{3}} \mathrm{e}_{a b c d)}^{*}=0,
$$

where the dots indicate operators of the form $D_{a_{4} b_{4}}$ and, apart from their relative positions, the positions of the operators $D_{e f}, D^{e f}$ are arbitrary. Thus we get

$$
\mathrm{e}_{p, 1: b_{p} c_{p} \cdots b_{3} c_{3} a b c d}^{\prime 0}=0 .
$$

It follows from (4.39) and (4.41) that $e_{a b c d}^{\prime}$ is of type $p-1$ and this holds true for $\breve{\phi}_{a b c d}^{\prime}$ since it is obtained from $e_{a b c d}^{\prime}$ by multiplying with a function of type $p$. This proves the first assertion of Theorem 4.1. Moreover, it follows now in terms of expansion (3.30) that 


$$
\begin{aligned}
& \left.\partial_{\rho}^{p} e_{a b c d}^{\prime}\right|_{\rho=0}=p !(\sqrt{2})^{p}\left\{s^{b_{p}}{ }_{0} s^{c_{p}}{ }_{1} \cdots s^{b_{1}}{ }_{0} s^{c_{1}}{ }_{1} \mathrm{e}_{p, 0 ;\left(b_{p} c_{p} \cdots b_{2}\right.}^{\prime 3}{ }^{\left(e_{\epsilon_{2}}\right.} \epsilon_{c_{2}}{ }^{\epsilon_{\epsilon_{1}}}{ }^{g} \epsilon_{\left.c_{1}\right)}{ }^{h)}\right. \\
& \left.-\frac{1}{2} s^{b_{p}}{ }_{0} s^{c_{p}}{ }_{1} \cdots s^{b_{3}}{ }_{0} s^{c_{3}}{ }_{1} \mathrm{e}_{p, 1 ;\left(b_{p} c_{p} \cdots b_{3}\right.}{ }^{\left(e f g_{\epsilon_{(3)}}{ }^{h)}\right\}}\right\} \\
& \times s_{e a} s_{f b} s_{g c} s_{h d}+\cdots,
\end{aligned}
$$

where the dots at the end of the right-hand side indicate terms of type lower than $p-1$. Since an odd number of $\epsilon$ 's occur in both terms written on the right-hand side and since multiplication by powers of $(U+\rho W)$ does not affect the asymmetry, we obtain (4.6).

To see that (4.7) is a necessary condition for (4.8) to be true, we show the following lemma:

\section{Lemma 4.6.}

$$
\begin{aligned}
\left.\partial_{\rho}^{3} e_{0000}^{\prime}\right|_{\rho=0}=c_{3} \sum_{k=0}^{4}\left(\begin{array}{l}
4 \\
k
\end{array}\right)^{1 / 2} b_{\left(a_{1} \cdots a_{4}\right)_{k}}^{*} T_{4}{ }^{k} 0, \quad c_{3}>0, \\
\left.\partial_{p}^{p} e_{0000}^{\prime}\right|_{\rho=0}=c_{p} \sum_{k=0}^{2(p-1)}\left(\begin{array}{c}
2(p-1) \\
k
\end{array}\right)^{1 / 2}\left(\begin{array}{c}
2(p-1) \\
p-3
\end{array}\right)^{-1 / 2} \\
\quad \times D_{\left(b_{p-3} c_{p-3} \cdots D_{b_{1} c_{l}} b_{\left.a_{1} \cdots a_{4}\right)_{k}}^{*} T_{2(p-1)}{ }^{k} p-3,\right.}, \quad p \geq 4 .
\end{aligned}
$$

That the first term of (4.38) is of the type as asserted by Lemma (4.6) follows from (4.40) and (4.38). It remains to determine the value of

$$
\mathrm{e}_{p, 1 ; b_{p} c_{p} \cdots a_{3} a b c}^{\prime 1}=\frac{4(p-2)}{p} \mathrm{e}_{p, 1 ;\left(a_{p} b_{p} \cdots b_{3} u_{3}|f| a b c\right)}^{*} f .
$$

Observing the expression of $e_{0111}^{\prime}$ given in (4.36) and property (ii) of Lemma 4.5, we find by taking for $p \geq 3$ the limit to $i$ of $D_{00}^{p-3} D_{0 f} \Delta e_{000}^{\prime}{ }^{f}$ that

$$
D_{\left(b_{p} c_{p}\right.} \cdots D_{b_{4} c_{4}} D_{a|f|} \mathrm{e}_{b c d)}^{{ }^{*}} f=p ! \frac{4 p^{2}-2 p-4}{p+1} D_{\left(b_{p} c_{p}\right.} \cdots D_{b_{4} c_{4}} b_{a b c d)}^{*} .
$$

By (iii) and (iv) of Lemma 4.5 and (4.38) the spinor $\mathrm{e}_{p .1 ; b_{p} c_{p} \ldots . a_{3} a b c}$ is sum of such terms with positive coefficients. Then the second term of (4.38) is also of the type described in Lemma 4.6. Assertion (iii) of Theorem 4.1 follows now.

\subsection{Expansion types and coefficients of various fields}

For later use we calculate the first few expansion coefficients.

\section{Lemma 4.7.}

(i) Assuming the cn-gauge, we find the following expansion types:

$$
\begin{aligned}
& \operatorname{type}(r)=\operatorname{type}(W)=p, \quad \operatorname{type}\left(s_{a b c d}\right)=p+1, \\
& \operatorname{type}\left(\check{\gamma}_{a b c d}\right)=p, \quad \operatorname{type}\left(\check{c}_{a b}^{ \pm}\right)=p, \quad \operatorname{type}(U-1)=p-1 .
\end{aligned}
$$


(ii) If for some non-negative integer $k$ it holds in addition

$$
D_{\left(b_{p} c_{p}\right.} \cdots D_{b_{1} c_{1}} b_{\left.a_{1} \cdots a_{4}\right)}^{*}=0, \quad p=0,1, \cdots, k
$$

then

$$
\begin{aligned}
& \operatorname{type}\left(\left.\partial_{\rho}^{p} s_{a b c d}\right|_{\rho=0}\right)=p, \quad p=0,1, \ldots, k+1, \\
& \operatorname{type}\left(\left.\partial_{\rho}^{p} \check{\gamma}_{a b c d}\right|_{\rho=0}\right)=p-1, \\
& \operatorname{type}\left(\left.\partial_{\rho}^{p} \check{c}_{a b}^{ \pm}\right|_{\rho=0}\right)=p-1, \\
& \operatorname{type}\left(\left.\partial_{\rho}^{p}(U-1)\right|_{\rho=0}\right)=p-2, \quad p=0,1, \ldots, k+2 .
\end{aligned}
$$

The functions $r$ and $W$ have expansion type $p$ in any gauge. The expansion type of the trace-free part of the 3-Ricci tensor follows from (4.30) and the expansion types of the fields $\check{\gamma}_{a b c d}, \breve{c}_{a b}^{ \pm}$then follow inductively from the structure equations $(3.23),(3.24),(3.21)$ and (3.22).

Using (4.11) together with (2.24) and the expansion type given above for $\dot{\gamma}_{a b c d}$, the final assertion of (i) follows.

The first assertion of (ii) follows from (4.30) and Lemma 4.4. The remaining assertions are shown now by going through the steps considered above.

For later use we note some consequences of the cn-gauge and of condition (4.7). At the point $i$ we have in cn-gauge

$$
l_{j i}=0, \quad D_{(k} l_{j i)}=0, \quad D_{(l} D_{k} l_{j i)}=0, \quad D_{(m} D_{l} D_{k} l_{j i)}=0 . \quad \text { at } i .
$$

The first of these equations is implied immediately by (4.27). It implies in turn by (4.22) and (4.23) al $i$

$$
D_{\dot{x}} \dot{x}=0, \quad D_{\dot{x}} D_{\dot{x}} \dot{x}=0, \quad D_{\dot{x}} b=0 .
$$

Taking directional derivatives of (4.27) and evaluating at $i$, we obtain there

$$
\dot{x}^{k} \dot{x}^{j} \dot{x}^{i} D_{k} l_{j i}=0, \quad \dot{x}^{l} \dot{x}^{k} \dot{x}^{j} \dot{x}^{i} D_{l} D_{k} l_{j i}=0, \quad \dot{x}^{m} \dot{x}^{l} \dot{x}^{k} \dot{x}^{j} \dot{x}^{i} D_{m} D_{l} D_{k} l_{j i}=0
$$

for all allowed initial data, whence our result.

By taking traces, respectively, symmetrizing we get from $(4.44)$

$$
r^{*}=0, \quad D_{a b} r^{*}=0, \quad D_{a b} D_{c d} r^{*}=-\frac{4}{5} \Delta_{h} s_{a b c d}^{*} .
$$

We now determine the first few expansion coefficients for various fields in the cn-gauge. For any symmetric spatial spinor field $t_{a b c d}$ one has in general

$$
D_{a b} t_{c d e f}=D_{(a b} t_{c d e f)}-\frac{5}{6}\left(\epsilon_{a(c} D_{b}{ }^{g} t_{d e f) g}+\epsilon_{b(c} D_{a}{ }^{g} t_{d e f) g}\right)-\frac{3}{5} \epsilon_{a(c} D^{g h} t_{|g h| d e} \epsilon_{f) b} .
$$

In the case of $s_{a b c d}$ this gives by (4.31) and (4.45)

$$
D_{a b} s_{c d e f}^{*}=-\frac{5}{6}\left(\epsilon_{a(c} b_{b d e f)}^{*}+\epsilon_{b(c} b_{a d e f)}^{*}\right) .
$$

It follows that 


$$
\begin{aligned}
& s_{(a b c d)_{j}}=s_{j, 1} \rho+\mathrm{O}\left(|\rho|^{2}\right), \\
& s_{j, 1}=\sqrt{2} \frac{2-j}{3} \sum_{k=0}^{4}\left(\begin{array}{l}
4 \\
k
\end{array}\right)^{1 / 2}\left(\begin{array}{l}
4 \\
j
\end{array}\right)^{-1 / 2} b_{(a b c d)_{k}}^{*} T_{4}{ }^{k}{ }_{j}
\end{aligned}
$$

and

$$
r=\mathrm{O}\left(|\rho|^{2}\right)
$$

By Lemma 4.7 we have expansions

$$
\check{c}_{a b}^{ \pm}=\sum_{p=1}^{\infty} \rho^{p} \sum_{q=q_{a b}^{ \pm}}^{p} \sum_{k=0}^{2 q} \stackrel{c}{ \pm}_{a b ; p: 2 q, k}^{ \pm} T_{2 q}^{k}{ }_{q \pm 1-1+a+b}
$$

with certain non-negative integers $q_{a b}^{ \pm}$, and

$$
\check{\gamma}_{a b c d}=\sum_{p=1}^{\infty} \rho^{p} \sum_{q=\max \{|2-a-b-c-d|, 1-p\}}^{p} \sum_{k=0}^{2 q} \check{\gamma}_{a b c d ; p ; 2 q, k} T_{2 q}{ }^{k}{ }_{q-2+a+b+c+d} .
$$

From the structure equations and the results above we get for $j=0,1,2$

$$
\begin{aligned}
& \check{c}_{(a b)_{j}}^{-}=\frac{1}{12}(1-j) s_{j, 1} \rho^{2}+\mathrm{O}\left(|\rho|^{3}\right), \\
& \check{c}_{(a b)_{j}}^{+}=-\frac{1}{12}(1-j) s_{j+2.1} \rho^{2}+\mathrm{O}\left(|\rho|^{3}\right), \\
& \check{\gamma}_{00(c d)_{j}}=-\frac{\sqrt{2}(4-|1-j|)}{24} s_{j, 1} \rho^{2}+\mathrm{O}\left(|\rho|^{3}\right), \\
& \check{\gamma}_{11(c d)_{j}}=\frac{\sqrt{2}(4-|1-j|)}{24} s_{j+2,1} \rho^{2}+\mathrm{O}\left(|\rho|^{3}\right) .
\end{aligned}
$$

If in addition to the cn-gauge condition (4.7) is satisfied, we have

$$
s_{i j}=\mathrm{O}\left(|\rho|^{2}\right), \quad \check{c}_{(a b)_{j}}^{ \pm}=\mathrm{O}\left(|\rho|^{3}\right), \quad \check{\gamma}_{a b c d}=\mathrm{O}\left(|\rho|^{3}\right) .
$$

Using the expression above in formula (4.11) we find that $U_{0}=1+\mathrm{O}\left(|\rho|^{4}\right)$. Observing this in the recursive definition of the function $U_{p+1}$ we find $U_{1}=\mathrm{O}\left(|\rho|^{2}\right)$ whence

$$
D_{a b} U(i)=0, \quad D_{c d} D_{a b} U(i)=0, \quad D_{e f} D_{c d} D_{a b} U(i)=0 .
$$

For the field $\Gamma^{2} d_{j k}^{\prime}=\Gamma^{2} \Omega^{\prime}-2 e_{j k}^{\prime}=U^{4} e_{j k}^{\prime}$ we get

$$
D_{\alpha}\left(\Gamma^{2} d_{k j}^{\prime}\right)(i)=D_{\alpha}\left(U^{4} e_{j k}^{\prime}\right)(i)=D_{\alpha} e_{k j}^{\prime}(i), \quad|\alpha| \leq 6 .
$$

The expansion type of the analytic lift of the tensor field (2.37) follows now immediately from the equation

$$
\Gamma A_{j k}=\Gamma\left\{-\Omega d_{j k}+s_{j k}+\frac{1}{12} r h_{j k}\right\} .
$$

The expansion of $D_{a b} \Omega$ can be derived easily from the data given above and the formula 


$$
\begin{aligned}
D_{a b} \Omega & =\frac{2 \rho x_{a b}}{(U+\rho W)^{2}}-2 \Gamma \frac{D_{a b} U+x_{a b} W+\rho D_{a b} W}{(U+\rho W)^{3}} \\
& =\frac{2 \rho\left(x_{a b} U-\rho D_{a b} U-\Gamma D_{a b} W\right)}{(U+\rho W)^{3}}=\frac{2 \rho\left(x_{a b}-\Gamma D_{a b} W\right)}{(1+\rho W)^{3}}+\mathrm{O}\left(|\rho|^{5}\right) .
\end{aligned}
$$

The expansions above entail

$$
\begin{aligned}
\breve{\phi}_{a b c d}^{W}=-\kappa^{3}\{ & 12 W \epsilon_{a b c d}^{2}+12 \rho x_{(a b} D_{c d)} W+2 \rho^{2} D_{(a b} D_{c d)} W \\
& -2 \rho^{2} W s_{a b c d}+2 \rho^{3} W D_{(a b} D_{c d)} W \\
& \left.-6 \rho^{3} D_{(a b} D_{c d)} W+\mathrm{O}\left(|\rho|^{4}\right)\right\} .
\end{aligned}
$$

Assuming that $W$ is an arbitrary solution to $\left(\Delta-\frac{1}{8} r\right) W=0$ on $B_{a}(i)$, we get

$$
W=\sum_{p=0}^{2} \rho^{p} W_{p}+\mathrm{O}\left(\rho^{3}\right)=\sum_{p=0}^{2} \rho^{p}\left(\sum_{k=0}^{2 p} W_{p ; 2 p, k} T_{2 p}{ }^{k}{ }_{p}\right)+\mathrm{O}\left(\rho^{3}\right)
$$

with

$$
\begin{aligned}
& W_{0 ; 0.0}=W^{*}=\frac{m}{2}, \quad W_{1 ; 2, k}=\left(\begin{array}{l}
2 \\
k
\end{array}\right)^{1 / 2} D_{(a b)_{k}} W^{*} . \\
& W_{2 ; 4, k}=\left(\begin{array}{l}
4 \\
2
\end{array}\right)^{-1 / 2}\left(\begin{array}{l}
4 \\
k
\end{array}\right)^{1 / 2} D_{(a b} D_{(d)_{k}} W^{*} .
\end{aligned}
$$

Furthermore

$$
\begin{aligned}
& D_{a b} W=\sum_{p=0}^{1} \rho^{p}\left\{x_{a b}(p+1) W_{p+1}+z_{a b} X_{+} W_{p+1}+y_{a b} X_{-} W_{p+1}\right\}+\mathrm{O}\left(|\rho|^{2}\right) . \\
& D_{(a b} D_{c d)_{j}} W=\sum_{k=0}^{4}\left(\begin{array}{l}
4 \\
2
\end{array}\right)^{1 / 2}\left(\begin{array}{l}
4 \\
j
\end{array}\right)^{-1 / 2} W_{2: 4, k} T_{4}{ }^{k} j+\mathrm{O}(|\rho|) .
\end{aligned}
$$

This gives the representation

$$
\begin{aligned}
\check{\phi}_{(a b c d)_{j}=-\kappa^{\prime}{ }^{3}\{} \delta^{2}{ }_{j}\left(2 W_{0}+6 \rho W_{1}+12 \rho^{2} W_{2}\right) \\
+\delta^{1}{ }_{j}\left(3 \rho X_{+} W_{1}+4 \rho^{2} X_{+} W_{2}\right) \\
+\delta^{3}{ }_{j}\left(3 \rho\left(-X_{-}\right) W_{1}+4 \rho^{2}\left(-X_{-}\right) W_{2}\right) \\
\left.+\delta^{0}{ }_{j} \rho^{2} X_{+} X_{+} W_{2}+\delta^{4}{ }_{j} \rho^{2} X_{-} X_{-} W_{2}+\mathrm{O}\left(|\rho|^{3}\right)\right\}
\end{aligned}
$$

Finally we have by (1.16)

$$
\breve{\phi}_{(a b c d)_{j}}^{\prime}=\kappa^{\prime 3}\left\{(2-j) \frac{\sqrt{2}}{6} \sum_{k=0}^{4}\left(\begin{array}{l}
4 \\
k
\end{array}\right)^{1 / 2} b_{(a b c d)_{k}}^{*} T_{4}{ }^{k}{ }_{j} \rho^{2}+\mathrm{O}\left(|\rho|^{3}\right)\right\} .
$$




\section{The evolution equations}

Our final goal is to gain control on the evolution of the fields in a neighbourhood of space-like infinity which extends to future and past null infinity. This problem, to which we refer as the "initial value problem near space-like infinity", will be given a more precise formulation at the end of this section. The intention is not only to show that the outgoing null geodesics starting close to space-like infinity are complete but also to analyse under which assumptions on the data the solution will allow a smooth conformal extension through null infinity. This requires precise information on the solution and leaves little freedom for playing with estimates. The main purpose of this section is to develop a formulation of the initial value problem which allows to relate properties of the initial data directly to properties of the fields near null infinity.

In space-times admitting smooth conformal extensions through null infinity the asymptotic behaviour of fields is analysed, "locally" near null infinity, in a convenient way in terms of coordinates based on outgoing null geodesics. Such coordinates are well adapted to the situation because null geodesics, considered as point sets, are invariant under conformal rescalings and their affine parameters have a simple transformation law. To analyse the gravitational field near space-like and null infinity in a time-symmetric way, one might therefore be tempted to use double null coordinates. However, already in the simplest non-trivial case, namely Schwarzschild space-time, such coordinates have undesirable properties. While the outgoing null hypersurfaces orthogonal to the orbits of the rotation group extend smoothly through future null infinity, the family of ingoing null hypersurfaces orthogonal to these orbits does not extend smoothly through future null infinity.

To avoid such problems, we shall employ gauge conditions based on time-like conformal geodesics. These curves are autoparallel with respect to "conformal connections", i.e. torsion free connections which preserve the conformal structure but not necessarily a metric in the conformal class. Thus we are forced to extend the analysis of conformal structures to include conformal connections. Such an analysis, including a detailed study of the use of conformal geodesics in the context of the field equations, has been carried out in [12].

It turns out that in terms of gauge conditions based on conformal geodesics the conformal factor can be determined explicitly in terms of the initial data. Thus, provided the fields and the congruence of conformal geodesics extend regularly to null infinity, we have near spacelike infinity perfect control on the location of null infinity. Furthermore, the newly acquired gauge freedom leads to a particularly simple conformal representation of the field equations.

In the usual conformal representation of the solution, where space-like infinity is thought of as a point, the initial value problem near space-like infinity, if formulated in terms of the conformal field equations, is a problem which is local but singular at space-like infinity (cf. (2.42) and (2.43)). By making full use of the formalism introduced in [12], this local singular problem can be converted into another problem where null infinity is represented by certain explicitly known hypersurfaces and where the data are regular at space-like infinity. The formulation of this "finite regular problem" will be the main result of this section.

In the following considerations we shall make use of the results of [12]. The reader is referred to this article for further details and the general background underlying our 
analysis. It should be noticed, however, that instead of using the conventions for the sign of the curvature spinors employed in [12] we shall adopt the convention of $[7,8,11]$.

\subsection{The conformal representation of the Einstein equations}

Suppose that $(\tilde{M}, \tilde{g})$ is a solution to the Einstein equations $(2.1)$ which contains the "physical part" $\tilde{S}=S \backslash\{\mathrm{i}\}$ of the 3-manifold $S$ as a smoothly embedded space-like Cauchy hypersurface such that the interior metric induced on $\tilde{S}$ is given by $\tilde{h}$ and the second fundamental form on $\tilde{S}$ vanishes. We shall be concerned here mainly with a detailed study of the evolution of the data on the punctured neighbourhood $B_{a}$ of $i$ in $S$.

Besides the metric $\tilde{g}$ and its Levi-Civita connection $\tilde{\nabla}$ the analysis will involve:

(i) A conformal connection $\hat{\nabla}$, i.e. a torsion free connection for which parallel transport preserves the $\tilde{g}$-causal nature of vectors.

(ii) A metric $g$ in the conformal class of $\tilde{g}$

$$
g=\Theta^{2} \tilde{g}
$$

(iii) The Levi-Civita connection $\nabla$ associated with $g$.

With any 1 -form $d$ on $\tilde{M}$ we associate the tensor field $S(d)$ given in index notation by

$$
S(d)_{\mu}{ }^{v}{ }_{\rho}=\delta^{v}{ }_{\mu} d_{\rho}+\delta^{v}{ }_{\rho} d_{\mu}-g_{\mu \rho} g^{\nu \lambda} d_{\lambda}
$$

Then, since the three connections considered above respect the same conformal structure, there are 1-forms $b$ and $f$ on $\tilde{M}$ such that the difference tensors of the connections are of the form

$$
\begin{aligned}
& \hat{\nabla}-\tilde{\nabla}=S(b), \quad \hat{\nabla}-\nabla=S(f), \\
& \nabla-\tilde{\nabla}=S(b-f)=S\left(\Theta^{-1} \mathrm{~d} \Theta\right) .
\end{aligned}
$$

We shall express the conformal field equations in terms of the conformal factor $\Theta$, the 1 -form $d=\Theta b$, the metric $g$, the 1 -form $f$, the connection $\hat{\nabla}$, and fields derived from these structures. Later on we shall express $\hat{\nabla}$ in terms of $\nabla$ and $f$. Let $S L(\tilde{M})$ denote the bundle of normalized spin frames over $\tilde{M}$ with structure group $S L(2 . C)$ and assume that the metric $g$ has spinor representation $g_{a a^{\prime} b b^{\prime}}=\epsilon_{a b} \epsilon_{a^{\prime} b^{\prime}}$ where $\epsilon_{01}=1$. As described in [12] we denote by $\sigma^{a a^{\prime}}$ the solder form on $S L(\tilde{M})$ and by $\omega^{a}{ }_{b}$ the $\operatorname{sl}(2, C)$-valued connection form representing the connection $\nabla$ on $S L(\tilde{M})$.

To accomodate the connection $\hat{\nabla}$ we have to consider an extended bundle. In an obvious way $S L(2, C)$ will be regarded as subgroup of the product of groups $C S L(2, C)=\mathbb{R}^{+} \times$ $S L(2, C)$. We denote by $C S L(\tilde{M})$ the associated bundle of fibre type $C S L(2, C)$. This principal fibre bundle may be identified with the set of the spin frames $\left\{\lambda \delta_{a}\right\}_{a}=0.1$ with $\left\{\delta_{a}\right\}_{a=0.1}$ in $S L(\tilde{M}), \lambda \in \mathbb{R}^{+}$. We denote by $\pi$ the projection of $\operatorname{CSL}(\tilde{M})$ onto $M$. The solder form $\sigma^{a a^{\prime}}$ and the connection form $\omega^{a}{ }_{b}$ extend in a natural way to $\operatorname{CSL}(\tilde{M})$. The connection $\hat{\nabla}$ is represented on $C S L(\tilde{M})$ by a $\mathbb{R} \oplus s l(2, C)$-valued connection form $\hat{\omega}^{a}{ }_{h}$ which is related to $\omega^{a}{ }_{b}$ by

$$
\hat{\omega}_{b}^{a}=\omega_{b}^{a}+f_{b c^{\prime}} \sigma^{a c^{\prime}} \text {. }
$$


where $f_{a a^{\prime}}$ denotes the invariant function on $C S L(\tilde{M})$ which represents the 1 -form $f$. The pull back of this 1-form to $C S L(\tilde{M})$ is thus given by $\hat{\omega} \equiv \hat{\omega}^{a}{ }_{a}=f_{a a^{\prime}} \sigma^{a a^{\prime}}$. We write $\Theta$ also for the pull back of $\Theta$ to $C S L(\tilde{M})$ and denote by $d_{a a^{\prime}}, \phi_{a b c d}, \Theta_{a a^{\prime} b b^{\prime}}$ the invariant functions on $C S L(\tilde{M})$ which represent the 1 -form $d$, the rescaled Weyl spinor field, and the tensor field (2.34), respectively. We have a decomposition

$$
\Theta_{a a^{\prime} b b^{\prime}}=\Phi_{a a^{\prime} b b^{\prime}}+\Lambda \epsilon_{a b} \epsilon_{a^{\prime} b^{\prime}}+\Phi_{a b} \epsilon_{a^{\prime} b^{\prime}}+\bar{\Phi}_{a^{\prime} b^{\prime}} \epsilon_{a b}
$$

where $2 \Phi_{a a^{\prime} b b^{\prime}}$ represents the symmetric trace-free part of the Ricci tensor $\hat{R}_{j k}$ of $\hat{\nabla}, \Lambda=$ $\frac{1}{24} g^{j k} \hat{R}_{j k}$, and the last two terms, with $\Phi_{a b}=\Phi_{(a b)}$, represent the antisymmetric tensor $\frac{1}{4} \hat{R}_{[j k]}$. For conciseness we write the field equations in terms of $\Theta_{a a^{\prime} b b^{\prime}}$. Finally we set

$$
K_{a a^{\prime} b b^{\prime} c c^{\prime}}=-d^{e e^{\prime}}\left(\phi_{e a b c} \epsilon_{e^{\prime} a^{\prime}} \epsilon_{b^{\prime} c^{\prime}}+\bar{\phi}_{e^{\prime} a^{\prime} b^{\prime} c^{\prime}} \epsilon_{e a} \epsilon_{b c}\right)
$$

and define the forms

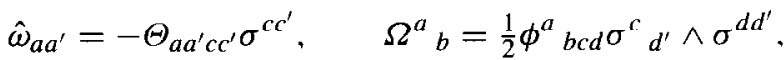

$$
\begin{aligned}
& \Omega_{a a^{\prime}}=\frac{1}{2} K_{a a^{\prime} b b^{\prime} c c^{\prime}} \sigma^{b b^{\prime}} \wedge \sigma^{c c^{\prime}} \text {. }
\end{aligned}
$$

Then, observing relation (5.3), the Einstein equations (2.1) are represented in terms of the conformal fields introduced above by the equations (cf. [12])

$$
\begin{aligned}
& 0=\mathrm{d} \sigma^{a a^{\prime}}+\omega^{a}{ }_{b} \wedge \sigma^{b a^{\prime}}+\bar{\omega}^{a^{\prime}}{ }_{b^{\prime}} \wedge \sigma^{a b^{\prime}}, \\
& 0=\mathrm{d} \hat{\omega}^{a}{ }_{b}+\hat{\omega}^{a}{ }_{c} \wedge \hat{\omega}^{c}{ }_{b}-\hat{\omega}_{b c^{\prime}} \wedge \sigma^{a c^{\prime}}-\Theta \Omega^{a}{ }_{b}, \\
& 0=\mathrm{d} \hat{\omega}-\hat{\omega}_{c c^{\prime}} \wedge \sigma^{c c^{\prime}}, \\
& 0=\mathrm{d} \hat{\omega}_{a a^{\prime}}+\hat{\omega}_{a c^{\prime}} \wedge \overline{\hat{\omega}}^{c^{\prime}}{ }_{a^{\prime}}+\hat{\omega}_{c a^{\prime}} \wedge \hat{\omega}^{c}{ }_{a}-\Omega_{a a^{\prime}}, \\
& 0=\mathrm{d} \Omega^{a}{ }_{b}-\Omega^{a}{ }_{c} \wedge \omega^{c}{ }_{b}+\omega^{a}{ }_{c} \wedge \Omega^{c}{ }_{b} .
\end{aligned}
$$

Eq. (5.6) is the contraction of (5.5), it has been added to the list for later convenience.

To write this system in the usual form of a differential system we choose a local section $\sigma: U \rightarrow \pi^{-1}(U)$, with $U$ an open subset of $\tilde{M}$, and define vector fields $c_{a a^{\prime}}$ on $\sigma(U)$ by the requirement

$$
\left\langle\sigma^{a a^{\prime}}, c_{b b^{\prime}}\right\rangle=\epsilon_{b}{ }^{a} \epsilon_{b^{\prime}} a^{\prime} .
$$

If we denote the pull back of $g$ to $\sigma(U)$ again by $g$, we then have

$$
g\left(c_{a a^{\prime}}, c_{b b^{\prime}}\right)=\epsilon_{a b} \epsilon_{a^{\prime} b^{\prime}} .
$$

We define connection coefficients on $\sigma(U)$ by

$$
\Gamma_{e e^{\prime}}{ }^{a}{ }_{b}=\left\langle\omega^{a}{ }_{b}, c_{e e^{\prime}}\right\rangle, \quad \hat{\Gamma}_{e e^{\prime}}^{a}{ }_{b}=\left\langle\hat{\omega}^{a}{ }_{b}, c_{e e^{\prime}}\right\rangle .
$$

They satisfy

$$
\Gamma_{c c^{\prime} a b}=\Gamma_{c c^{\prime} b a}, \quad \hat{\Gamma}_{c c^{\prime}}{ }^{a}{ }_{b}=\Gamma_{c c^{\prime}}{ }^{a}{ }_{b}+\epsilon_{c}{ }^{a} f_{b c^{\prime}} .
$$

The connection coefficients which occur in tensor calculations are then given by 


$$
\begin{aligned}
& \Gamma_{b b^{\prime}} a a^{\prime}{ }_{c c^{\prime}}=\Gamma_{b b^{\prime}}{ }^{a}{ }_{c} \epsilon_{c^{\prime}}{ }^{a^{\prime}}+\bar{\Gamma}_{b b^{\prime}}{ }^{a^{\prime}}{ }_{c^{\prime}} \epsilon_{c}{ }^{a}, \\
& \hat{\Gamma}_{b b^{\prime}} a a^{\prime}{ }_{c c^{\prime}}=\hat{\Gamma}_{b b^{\prime}}{ }^{a}{ }_{c} \epsilon_{c^{\prime}}{ }^{a^{\prime}}+\overline{\hat{\Gamma}}_{b b^{\prime}}{ }^{a^{\prime}}{ }_{c^{\prime}} \epsilon_{c}{ }^{a} .
\end{aligned}
$$

Using the connection coefficients we define on $\sigma(U)$ covariant derivatives of spinor-valued functions by the usual formulas and denote by $\nabla_{a a^{\prime}}$ resp. $\hat{\nabla}_{a a^{\prime}}$ covariant derivatives in the direction of $c_{a a^{\prime}}$. Evaluation of the system of forms on the vector fields $c_{a a^{\prime}}$ now gives the system of partial differential equations

$$
\begin{aligned}
& 0=\left[c_{b b^{\prime}}, c_{c c^{\prime}}\right]-\left(\Gamma_{b b^{\prime}}{ }^{a a^{\prime}} c_{c c^{\prime}}-\Gamma_{c c^{\prime}} a a^{\prime} b b^{\prime}\right) c_{a a^{\prime}} . \\
& 0=c_{c c^{\prime}}\left(\hat{\Gamma}_{d d^{\prime}}{ }^{a}{ }_{b}\right)-c_{d d^{\prime}}\left(\hat{\Gamma}_{c c^{\prime}}{ }^{a}{ }_{b}\right) \\
& -\hat{\Gamma}_{c c^{\prime}}{ }^{f}{ }_{d} \hat{\Gamma}_{f d^{\prime}}{ }^{a}{ }_{b}+\hat{\Gamma}_{d d^{\prime}}{ }^{f}{ }_{c} \hat{\Gamma}_{f c^{\prime}}{ }^{a}{ }_{b}-\overline{\hat{\Gamma}}_{c c^{\prime}} f^{\prime}{ }_{d^{\prime}} \hat{\Gamma}_{d f^{\prime}}{ }^{a}{ }_{b} \\
& +\overline{\hat{\Gamma}}_{d d^{\prime}} f^{\prime}{ }_{c^{\prime}} \hat{\Gamma}_{c f^{\prime}}{ }_{b}{ }_{b}+\hat{\Gamma}_{c c^{\prime}}{ }^{a}{ }_{f} \hat{\Gamma}_{d d^{\prime}} f_{b}-\hat{\Gamma}_{d d^{\prime}}{ }^{a}{ }_{f} \hat{\Gamma}_{c c^{\prime}}{ }^{f}{ }_{b}
\end{aligned}
$$

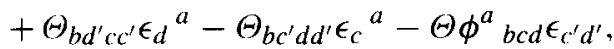

$$
\begin{aligned}
& 0=\hat{\nabla}_{c c^{\prime}}\left(f_{d d^{\prime}}\right) \quad \hat{\nabla}_{d d^{\prime}}\left(f_{c c^{\prime}}\right)+\Theta_{d d^{\prime} c c^{\prime}}-\Theta_{c^{\prime} d d^{\prime}}, \\
& 0=\hat{\nabla}_{b b^{\prime}} \Theta_{c c^{\prime} a a^{\prime}}-\hat{\nabla}_{a a^{\prime}} \Theta_{c c^{\prime} b b^{\prime}}-d^{e e^{\prime}}\left(\phi_{e a b c} \epsilon_{e^{\prime} c^{\prime}} \epsilon_{a^{\prime} b^{\prime}}+\bar{\phi}_{e^{\prime} a^{\prime} b^{\prime} c^{\prime}} \epsilon_{e c} \epsilon_{a b}\right) \text {, } \\
& 0=\nabla^{h} \cdot \phi_{a b c h}
\end{aligned}
$$

for the unknowns

$$
c_{a a^{\prime}}, \quad \hat{\Gamma}_{a a^{\prime} b c}, \quad f_{a a^{\prime}}=\hat{\Gamma}_{a a^{\prime}}{ }_{b}, \quad \Theta_{a a^{\prime} b b^{\prime}}, \quad \phi_{a b c d} .
$$

We note that the fields $\Theta, b_{a a^{\prime}}$ are not subject to differential equations here. Their occurrence reflects the conformal gauge freedom introduced into the Einstein equations.

\subsection{Gauge conditions}

To fix the gauge dependent quantities, we consider solutions $x^{\mu}(\tau), b_{v}(\tau), \mathrm{e}_{k}^{\lambda}(\tau)$ to the conformal geodesics equations

$$
\begin{aligned}
& \tilde{\nabla}_{\dot{x}} \dot{x}=-2\langle b, \dot{x}\rangle \dot{x}+\tilde{g}(\dot{x}, \dot{x}) \tilde{g}^{\#}(b, \cdot), \\
& \tilde{\nabla}_{\dot{x}} b=\langle b, \dot{x}\rangle b-\frac{1}{2} \tilde{g}^{\#}(b, b) \tilde{g}(\dot{x}, \cdot)+\tilde{L}(\dot{x}, \cdot), \\
& \tilde{\nabla}_{\dot{x}} \mathrm{e}_{k}=-\left\langle b, \mathrm{e}_{k}\right\rangle \dot{x}-\langle h, \dot{x}\rangle \mathrm{e}_{k}+\tilde{g}\left(\dot{x}, \mathrm{e}_{k}\right) b .
\end{aligned}
$$

Here $x^{\mu}(\tau)$ is a space-time curve in $\tilde{M}, b_{\nu}$ is a 1 -form, and $\mathrm{e}_{k}=\mathrm{e}_{k}^{\lambda} \partial_{\lambda}$ a frame field along this curve. The tensor field $\tilde{L}$, which is determined from the Ricci tensor of $\tilde{g}$, vanishes under our assumptions.

For given point $q \in \tilde{S}$ we consider conformal geodesics satisfying at that point initial conditions with

$$
\begin{aligned}
& x(0)=q, \quad \dot{x}(0)=\mathrm{e}_{0} \quad \text { is future directed and orthogonal to } \tilde{S}, \\
& \Theta_{*}^{2} \tilde{g}\left(\mathrm{e}_{j}, \mathrm{e}_{k}\right)=\eta_{j k}, \quad \Theta_{*}>0, \\
& \langle b, \dot{x}\rangle=0 .
\end{aligned}
$$

This leaves the freedom to specify on $\tilde{S}$ the function $\Theta_{*}$ and the pull back of $b$ to $\tilde{S}$. 
As shown in Lemmas 3.1 and 3.2 of [12], the solutions to the conformal geodesic equations for such initial conditions satisfy

$$
\dot{x}(\tau)=\mathrm{e}_{0}(\tau), \quad \Theta^{2} \tilde{g}\left(\mathrm{e}_{j}, \mathrm{e}_{k}\right)=\eta_{j k}
$$

with a conformal factor $\Theta=\Theta(\tau)$ such that

$$
\Theta(\tau)=\Theta_{*}\left(1-\tau^{2} \frac{\left|\tilde{h}^{\#}(b, b)\right|_{*}}{4 \Theta_{*}^{2}}\right), \quad d_{k}(\tau) \equiv \Theta b_{\mu} \mathrm{e}_{k}^{\mu}=\left(\dot{\Theta}, d_{a *}\right)
$$

Here, as below, quantities with lower index $*$ are assumed to be independent of $\tau$ such that along a conformal geodesic they are given by their value at the intersection of the conformal geodesics with $\tilde{S}$. We denote by $\tilde{h}^{\#}(b, b)$ the scalar product of the pull back of $b$ to $\tilde{S}$ with itself. On the right-hand side of the last equation we use vector notation, with $d_{a *}, a=1,2,3$, denoting the value of $\Theta_{*} b_{\mu} \mathrm{e}^{\mu}{ }_{a}$ at $p$.

In the following we denote by $\kappa$ some smooth, positive function on $\tilde{S}$. Specializing to

$$
\begin{aligned}
& \Theta_{*}=\kappa^{-1} \Omega \text { on } \tilde{S}, \\
& \quad \text { pull back of } b \text { to } \tilde{S}=\Omega^{-1} \mathrm{~d} \Omega,
\end{aligned}
$$

where $\Omega$ is the conformal factor determined in Section 2 and $\kappa$ some smooth function on $\tilde{S}$, we get

$$
\begin{aligned}
& \Theta(\tau)=\kappa_{*}^{-1} \Omega_{*}\left(1-\tau^{2} \frac{\kappa_{*}^{2}}{\omega_{*}^{2}}\right) \\
& d_{k}(\tau)=\left(-2 \tau \frac{\kappa_{*} \Omega_{*}}{\omega_{*}^{2}}, \kappa_{*}^{-1}\left(\mathrm{e}_{a}(\Omega)\right)_{*}\right)
\end{aligned}
$$

Here

$$
\omega=\frac{2 \Omega}{\sqrt{\left|D_{a} \Omega D^{a} \Omega\right|}}=\left(\frac{\Omega}{|\Delta \Omega / 6+r \Omega / 24|}\right)^{1 / 2} \quad \text { on } \tilde{S} \text { close to } i \text {. }
$$

Notice that, in accordance with assumption (2.12), the normal derivative of $\Theta$ vanishes on $\tilde{S}$.

The functions $\Theta$ and $b_{k}$ are known a priori and are given completely in terms of data on $\tilde{S}$. The components of the 1-form field $b$ in conformal Gauss coordinates are not known a priori since the frame coefficients $\mathrm{e}^{\mu}{ }_{k}$ are not known before the propagation equations have been solved.

For given $\kappa>0$ on $\tilde{S}$ conditions (5.20)-(5.24) determine on some neighbourhood $W$ of $\tilde{S}$ in $\tilde{M}$ a smooth congruence of conformal geodesics orthogonal to $\tilde{S}$ and a smooth time function $x^{0} \equiv \tau$ with $x^{0}=0$ on $\tilde{S}$. We assume, possibly after shrinking $\tilde{M}$, that $W$ coincides with $\tilde{M}$.

Local coordinates $x^{\alpha}, \alpha=1,2,3$, on $\tilde{S}$ can be dragged along the congruence to obtain coordinates $x^{0}, x^{\alpha}$ on $\tilde{M}$. Such coordinates will be referred to as "conformal Gauss coordinates". 
The 1-form $b_{\mu}$ is smooth near $\tilde{S}$ and defines a conformal connection $\hat{\nabla}$ by the first of Eqs. (5.2). Setting

$$
f_{\mu}=b_{\mu}-\Theta^{-1} \nabla_{\mu} \Theta
$$

the remaining equations of (5.2) are satisfied as well. Along the conformal geodesics we have

$$
\hat{\nabla}_{\dot{x}} \mathrm{e}_{k}=0, \quad \hat{\nabla}_{\dot{x}} g=0, \quad g\left(\mathrm{e}_{i}, \mathrm{e}_{j}\right)=\eta_{i j},
$$

and in conformal Gauss coordinates $x^{\mu}$ the frame coefficients $\mathrm{e}^{\mu}{ }_{k}=\left\langle\mathrm{d} x^{\mu}, \mathrm{e}_{k}\right\rangle$ satisfy

$$
\mathrm{e}^{\mu}{ }_{0}=\delta^{\mu}{ }_{0} \quad \text { on } \tilde{M} \text {. }
$$

In general $\mathrm{e}^{0}{ }_{j}=0$ for $j=1,2,3$ only on $\tilde{S}$.

We choose now a smooth spin frame field $\delta=\left\{\delta_{a}\right\}_{a=0.1}$ on $\tilde{M}$ which is parallely propagated along the congruence of conformal geodesics with respect to $\hat{\nabla}$ and which is such that $\delta_{a} \bar{\delta}_{a^{\prime}}=\mathrm{e}_{a a^{\prime}} \equiv \sigma^{k}{ }_{a a^{\prime}} \mathrm{e}_{k}$, where $\sigma_{a a^{\prime}}^{k}$ are the constant Van der Waerden symbols. This spin frame field defines a section of $\operatorname{CSL}(\tilde{M})$ which we take to be the section $\sigma$ used to derive Eqs. (5.11)-(5.15). It follows that the vectors $c_{a a^{\prime}}$ project onto the vectors $\mathrm{e}_{a a^{\prime}}$.

We will refer to the pull back of Gauss coordinates to $\sigma(\tilde{M})$ again as to Gauss coordinates. In such coordinates our constructions entails the relation

$$
\tau^{a a^{\prime}} \mathrm{e}_{a a^{\prime}}=\sqrt{2} \mathrm{e}_{0}=\sqrt{2} \partial_{\tau} \quad \text { with } \tau^{a a^{\prime}}=\epsilon_{0}{ }^{a} \epsilon_{0}{ }^{a^{\prime}}+\epsilon_{1}{ }^{a} \epsilon_{1}{ }^{\prime},
$$

whence also

$$
\tau^{a a^{\prime}} c^{\mu}{ }_{a a^{\prime}}=\sqrt{2} \delta^{\mu}{ }_{0}
$$

on $\tilde{M}$. As shown in [12], we have in our gauge also

$$
\tau^{a a^{\prime}} \hat{\Gamma}_{a a^{\prime} b c}=0, \quad \tau^{a a^{\prime}} f_{a a^{\prime}}=0, \quad \tau^{b b^{\prime}} \Theta_{a a^{\prime} b b^{\prime}}=0, \quad \text { on } \tilde{M} .
$$

Since in our gauge the functions $\Theta$ and $d_{a a^{\prime}}$ are known, the system (5.11)-(5.15) is in fact a differential system for the fields (5.16).

\subsection{The finite regular initial value problem near space-like infinity}

To formulate the desired initial value problem we extend the formalism introduced in Section 3 and assume the gauge described above. Thus we keep in particular the metric $g$, its Levi-Civita connection form $\omega^{a}{ }_{b}$, the connection $\hat{\nabla}$, the associated connection form $\hat{\omega}^{a}{ }_{b}$, the conformal factor $\Theta$, and the 1-form field $d_{a a^{\prime}}$ introduced in Section 5.2.

The metric $\kappa_{*}^{2} g$ is by our construction independent of the choice of $\kappa$. We associate with it the bundle $S L(\bar{M})$ of normalized spin frames considered earlier and denote by $S U(\tilde{S})$ its subset of spin frames which are defined over $\tilde{S}$ and satisfy the normalization condition (3.2) as well as (3.1). The subset $C_{a}^{+}$of $C_{a} \subset S U(S)$ on which $\rho$ is positive is a smooth submanifold of $S L(\tilde{S})$, thus also of $C S L(\tilde{M})$, which projects onto $B_{a}$. 
Consider the rescaling map $\delta \rightarrow \kappa^{1 / 2} \delta$ defined for spin frames $\delta$ over $B_{a}$. Its restriction to $C_{a}^{+}$(which we consider here only as a map of points) defines a diffeomorphism $\Sigma$ of $C_{a}^{+}$ onto a submanifold $C_{a, \kappa}^{+}=\kappa^{1 / 2} C_{a}^{+}$of $C S L(\tilde{M})$. We use this diffeomorphism to carry over to $C_{a, k}^{+}$the coordinates $\rho, t$ and the vector fields $\partial_{\rho}, X, X_{+}, X_{-}$defined on $C_{a}^{+}$in Section 3. The projection of $C_{a, k}^{+}$onto $B_{a}$ will be denoted again by $\pi^{\prime}$.

The connection form $\hat{\omega}^{a}{ }_{b}$ determines a vector field $\hat{H}$ on $\operatorname{CSL}(\tilde{M})$ by the conditions

$$
\left\langle\hat{\omega}^{a}{ }_{b}, \hat{H}\right\rangle=0, \quad\left\langle\sigma^{a a^{\prime}}, \hat{H}\right\rangle=\frac{1}{\sqrt{2}} \tau^{a a^{\prime}},
$$

i.e. $\hat{H}$ is the vector field which is horizontal with respect to the connection $\hat{\nabla}$ and has projection $T_{\delta}(\pi) \hat{H}(\delta)=(1 / \sqrt{2}) \tau^{a a^{\prime}} \delta_{a} \bar{\delta}_{a^{\prime}}$.

At points of $C_{a, \kappa}^{+}$the vector field $\hat{H}$ projects onto the future directed $g$-unit normal $\mathrm{c}_{0}$ of $B_{a}$ in $\tilde{M}$. The push forward of $C_{a, \kappa}^{+}$by $\hat{H}$ is a smooth five-dimensional submanifold of $\operatorname{CS} L(\tilde{M})$ which we denote by $M_{u, \kappa}^{+}$. The induced projection is again denoted by $\pi$ and its image, which is independent of the choice of $\kappa$, is denoted by $\tilde{M}_{a}$. Points on $M_{a, \kappa}^{+}$ are spin frames $\delta$ over $\tilde{M}_{a}$ which satisfy with respect to the metric $g$ the normalization $g\left(\delta_{a} \bar{\delta}_{a^{\prime}}, \delta_{b} \bar{\delta}_{b^{\prime}}\right)=\epsilon_{a b} \epsilon_{a^{\prime} b^{\prime}}$.

By our construction $M_{a, \kappa}^{+}$is a fibre bundle over $\tilde{M}_{a}^{+}$with fibre type $U(1)$. Denoting by $\tau$ the parameter on the flow lines of $\hat{H}$ which vanishes on $C_{a, \kappa}^{+}$, the projections of these curves coincide with the conformal geodesics considered in Section 5.2. Flow lines passing through the same fibre of $M_{a, k}^{+}$project onto the same conformal geodesic.

Dragging along with the flow of $\hat{H}$ the coordinates $\rho$ and $t$ given on $C_{a, \kappa}^{+}$, we obtain smooth coordinates $\tau, \rho, t$ on $M_{a . \kappa}^{+}$. The vector fields $\partial_{\rho}, X, X_{+}, X_{-}$on $C_{a . \kappa}^{+}$extend in a unique way to vector fields on $M_{a, \kappa}^{+}$having vanishing commutator with $\hat{H}$. Together with $\hat{H}=\partial_{\tau}$ these vector fields constitute a frame field on $M_{a, \kappa}^{+}$. The vector field i $X$ generates the fibres of $M_{a, \kappa}^{+}$. Close to $B_{a}$ the projections of the fields $\partial_{\tau}, \partial_{\rho}, X_{+}, X_{-}$generate the tangent spaces at points of $\tilde{M}_{a}$.

Using the cmbedding of $M_{a, k}^{+}$into $C S L(\tilde{M})$, wc can pull back to $M_{a, k}^{+}$forms and invariant functions as well as the equations considered on $\operatorname{CS} L(\tilde{M})$. Using for the quantities so obtained the same notation as before, we have forms $\sigma^{a a^{\prime}}, \hat{\omega}^{a}{ }_{b}, \omega^{a}{ }_{b}$ which we call again solder and connection form, respectively.

In analogy with our procedure on $C_{a}^{+}$we define vector fields $c_{a a^{\prime}}$ on $M_{a, k}^{+}$by the requirements

(i) $\left\langle\sigma^{a a^{\prime}}, c_{b b^{\prime}}\right\rangle=\epsilon_{b}{ }^{a} \epsilon_{b^{\prime}} a^{\prime}$.

(ii) $c_{a a^{\prime}}=c^{0}{ }_{a a^{\prime}} \partial_{\tau}+c^{1}{ }_{a a^{\prime}} \partial_{\rho}+c^{+}{ }_{a a^{\prime}} X_{+}+c^{-}{ }_{a a^{\prime}} X_{-}$.

Here the expansion in (ii), which excludes components in the direction of $X$, ensures the uniqueness of the vector fields. These vector fields allow splittings of the form

$$
c_{a a^{\prime}}=\frac{1}{2} \tau_{a a^{\prime}} \tau^{e e^{\prime}} c_{e e^{\prime}}-\tau^{b}{ }_{a^{\prime}} c_{a b}
$$

with $\tau^{a a^{\prime}} c_{a a^{\prime}}=\sqrt{2} \partial_{\tau}$, which is the analogue of (5.30), and vector fields

$$
c_{a b}=\tau_{(a}{ }^{b^{\prime}} c_{b) b^{\prime}}=c^{0}{ }_{a b} \partial_{\tau}+c_{a b}^{1} \partial_{\rho}+c^{+}{ }_{a b} X_{+}+c^{-}{ }_{a b} X_{-} .
$$


It should be observed that the spatial component of these vector fields are identical with the vector fields $c_{a b}$ introduced in Section 3 only for the choice $\kappa=1$.

To the splitting of the vector fields corresponds an expansion of the solder form

$$
\sigma^{a a^{\prime}}=\frac{1}{2} \tau^{a a^{\prime}} \tau_{c c^{\prime}} \sigma^{c c^{\prime}}+\tau_{c^{\prime}}{ }^{\prime} \sigma^{c a}
$$

with $\sigma^{a b}=-\tau^{(a} a^{\prime} \sigma^{b) a^{\prime}}$. We have

$$
\begin{aligned}
& \left\langle\tau_{c c^{\prime}} \sigma^{c c^{\prime}}, \tau^{e e^{\prime}} c_{e e^{\prime}}\right\rangle=2, \quad\left\langle\tau_{\left.c c^{\prime} \sigma^{c c^{\prime}}, c_{b d}\right\rangle=0 .}\right. \\
& \left\langle\sigma^{a c}, \tau^{e e^{\prime}} c_{e e^{\prime}}\right\rangle=0, \quad\left\langle\sigma^{a c}, c_{b d}\right\rangle=h^{a c} b d .
\end{aligned}
$$

Let $\alpha^{ \pm}, \alpha$ be the 1 -forms on $M_{a}^{+}$which annihilate the vector fields $\partial_{\tau}, \partial_{\rho}$ and have with $X_{ \pm}, X$ non-vanishing pairings

$$
\left\langle\alpha^{+}, X_{+}\right\rangle=\left\langle\alpha^{-}, X_{-}\right\rangle=\langle\alpha, X\rangle=1
$$

only. Since $X$ is vertical and the solder form is horizontal, the latter allows an expansion

$$
\sigma^{a a^{\prime}}=\sigma_{\tau}{ }^{a a^{\prime}} \mathrm{d} \tau+\sigma_{\rho}{ }^{a a^{\prime}} \mathrm{d} \rho+\sigma_{+}{ }^{a a^{\prime}} \alpha^{+}+\sigma_{-}{ }^{a a^{\prime}} \alpha^{-} \text {. }
$$

From the pairings above we conclude that

$$
\begin{aligned}
& \tau_{a a^{\prime} \sigma_{\tau} a a^{\prime}}=\sqrt{2}, \\
& \tau_{a a^{\prime} \sigma_{\rho}}{ }^{a a^{\prime}} c^{1}{ }^{\prime} b d+\tau_{a a^{\prime}} \sigma_{+}{ }^{a a^{\prime}} c^{+}{ }_{b d}+\tau_{a a^{\prime}} \sigma_{-}{ }^{a a^{\prime}} c^{-}{ }_{b d}+\sqrt{2} c^{0} b d=0
\end{aligned}
$$

and that the forms $\sigma^{a b}$ have expansions $\sigma^{a c}=\sigma_{\rho}{ }^{a c} d \rho+\sigma_{+}{ }^{a c} \alpha_{+}+\sigma^{-a c} \alpha^{-}$with coefficients salisying

$$
\sigma_{\rho}^{a c} c^{1}{ }_{b d}+\sigma_{+}{ }^{a c} c^{+}{ }_{b d}+\sigma_{-}{ }^{a c} c^{-}{ }_{b d}=h^{a c} b d .
$$

Given the vector fields $c_{a b}$, we can determine by the equations above the expansion coefficients of the solder form. The pull back of the metric to $M_{a}^{+}$, denoted again by $g$, is then given by

$$
g=\epsilon_{a b} \epsilon_{a^{\prime} b^{\prime}} \sigma^{a a^{\prime}} \sigma^{b b^{\prime}}=\frac{1}{2} \tau_{a a^{\prime}} \sigma^{a a^{\prime}} \tau_{b b^{\prime}} \sigma^{b b^{\prime}}+h_{a b c d} \sigma^{a b} \sigma^{c d} .
$$

Connection coefficients, which satisfy again (5.10), are defined on $M_{a, \kappa}^{+}$by formulae (5.9). Again, invariant functions induced on $M_{a, \kappa}^{+}$by covariant differentials of spinor fields are given then by the usual expressions for covariant derivatives in terms of the frame $c^{\prime} a u^{\prime}$ and of the connection coefficients. Relations (5.31) also hold on $M_{a, \kappa}^{+}$.

The conformal factor lifts to a function on $M_{a, \kappa}^{+}$which we denote again by $\Theta$. Using the expression $\Omega=\Gamma /(U+\rho W)^{2}$ we obtain for the function (5.26) appearing in (5.25)

$$
\begin{gathered}
\omega=\rho(U+\rho W)\left\{U^{2}+2 \rho U x^{a b} D_{a b} U-\rho^{2} D^{a b} U D_{a b} U+2 \rho^{2} U x^{a b} D_{a b} W\right. \\
\left.-2 \rho^{3} D^{a b} U D_{a b} W-\rho^{4} D^{a b} W D_{a b} W\right\}^{-1 / 2} .
\end{gathered}
$$

Using (5.25) we get for $d_{a b}=\tau_{(a} h^{\prime} d_{b) b^{\prime}}$ an expression which does not contain the factor $\kappa_{*}^{-1}$ explicitly

$$
d_{a b}=2 \rho\left(\frac{U x_{a b}-\rho D_{a b} U-\rho^{2} D_{a b} W}{(U+\rho W)^{3}}\right)_{*} .
$$




\subsubsection{The propagation equations}

Evaluation of the conformal Einstein equations (5.4)-(5.8) on $X \wedge c_{a a^{\prime}}$ gives equations which determine the spin weights of the unknowns (5.16). To obtain propagation equations we evaluate Eqs. (5.4)-(5.8) on $c_{a a^{\prime}} \wedge c_{b b^{\prime}}$. To obtain a suitable form, we follow the procedure in [12, Section 5], express the equations in terms of the 1-form $f$ and the connection $\nabla$. convert primed indices into unprimed indices, and introduce some useful notation. We set

$$
\begin{aligned}
& c_{a b}=\tau_{(b} b^{h^{\prime}} c_{a) b^{\prime}}, \quad \Gamma_{a b c d}=\tau_{b}{ }^{b^{\prime}} \Gamma_{a b^{\prime} c d}, \quad f_{a b}=\tau_{b}{ }^{b^{\prime}} f_{a b^{\prime}}, \\
& \sqrt{2} \chi_{a b c d} \equiv-\Gamma_{a b c d}-\Gamma^{+}{ }_{b a c d} \\
& \equiv-\Gamma_{a b c d}+\tau_{b}{ }^{b^{\prime}} \tau_{c}{ }^{c^{\prime}} \tau_{d} d^{\prime} \bar{\Gamma}_{a b^{\prime} c^{\prime} d^{\prime}} \\
& =\tau_{b}{ }^{a^{\prime}}\left(\nabla_{a a^{\prime}} \tau_{c c^{\prime}}\right) \tau_{d} c^{\prime} \text {, } \\
& \sqrt{2} \xi_{a b c d} \equiv \Gamma_{a b c d}-\Gamma^{+}{ }_{b a c d}, \quad \Theta_{a c b d}=\tau_{c}{ }^{a^{\prime}} \tau_{d}{ }^{b^{\prime}} \Theta_{a a^{\prime} b b^{\prime}} .
\end{aligned}
$$

From (5.31) follows

$$
f_{a b}=f_{(a b)}, \quad \Gamma_{c}{ }^{c} a b=\tau^{c c^{\prime}} \Gamma_{c c^{\prime} a b}=-f_{a b}, \quad \Theta_{a b c}{ }^{c}=0 .
$$

Since the connection $\nabla$ is metric we have

$$
\Gamma_{a b c d}=\Gamma_{a b(c d)}, \quad \chi_{a b c d}=\chi_{a b(c d)}, \quad \xi_{a b c d}=\xi_{(a b)(c d)}
$$

and thus

$$
\Gamma_{a b c d}=\frac{1}{\sqrt{2}}\left(\xi_{a b c d}-\chi_{a b c d}\right)=\frac{1}{\sqrt{2}}\left(\xi_{a b c d}-\chi_{(a b) c d}\right)-\frac{1}{2} \epsilon_{a b} f_{c d} .
$$

We use the covariant directional derivative operators

$$
\mathcal{D}_{a b}=\tau_{(a}{ }^{a^{\prime}} \nabla_{b) a^{\prime}}, \quad P=\tau^{a a^{\prime}} \nabla_{a a^{\prime}}
$$

to express the evolution equations as equations for

$$
c_{a b}^{\mu}, \quad \Gamma_{a b c d}, \quad \Theta_{a c b d}, \quad \phi_{a c b d} .
$$

and the derived quantities

$$
f_{a b}, \chi_{a b c d}, \quad \xi_{a b c d} .
$$

By suitable contractions with $\tau^{a a^{\prime}}$ we get from (5.11)-(5.14)

$$
\begin{aligned}
& \partial_{\tau} c^{0}{ }_{a b}=-\chi_{(a b)}{ }^{e f} c^{0}{ }_{e f}-f_{a b}, \\
& \partial_{\tau} c_{a b}^{\alpha}=-\chi_{(a b)}{ }^{e f} c^{\alpha}{ }_{e f}, \quad \alpha=1,2,3 \text {, } \\
& \partial_{\tau} \xi_{a b c d}=-\chi_{(a b)}{ }^{e f} \xi_{e f c d}+\frac{1}{\sqrt{2}}\left(\epsilon_{a c} \chi(b d) e f+\epsilon_{b d} \chi_{(a c) e f}\right) f^{e f} \\
& -\sqrt{2} \chi_{(a b)\left(c^{e}\right.} f_{d) e}-\frac{1}{2}\left(\epsilon_{a c} \Theta_{f} f_{b d}+\epsilon_{b d} \Theta_{f} f_{a c}\right)-\mathrm{i} \Theta \mu_{a b c d}, \\
& \partial_{\tau} f_{a b}=-\chi_{(a b)}{ }^{e f} f_{e f}+\frac{1}{\sqrt{2}} \Theta_{f} f_{a b} \text {, }
\end{aligned}
$$




$$
\begin{aligned}
& \partial_{\tau} \chi_{(a b) c d}=-\chi_{(a b)}{ }^{e f} \chi_{e f c d}-\Theta_{(c d) a b}+\Theta \eta_{a b c d}, \\
& \partial_{\tau} \Theta_{(a b) c d}=-\chi_{(c d)}{ }^{e f} \Theta_{(a b) e f}-\partial_{\tau} \Theta \eta_{a b c d}+\mathrm{i} \sqrt{2} d^{e}{ }_{(a} \mu_{b) c d e} \\
& \partial_{\tau} \Theta_{g}{ }^{g}{ }_{a b}=-\chi_{(a b)}{ }^{e f} \Theta_{g}{ }^{g}{ }_{e f}+\sqrt{2} d^{e f} \eta_{a b e f} .
\end{aligned}
$$

Herc expressions (5.25), (5.36) and (5.37) for $\Theta, \partial_{\tau} \Theta$ and $d_{a b}$ are assumed and we denote by $\eta_{a b c d}=\frac{1}{2}\left(\phi_{a b c d}+\phi_{a b c d}^{+}\right)$and $\mu_{a b c d}=-\frac{1}{2} \mathrm{i}\left(\phi_{a b c d}-\phi_{a b c d}^{+}\right)$the electric and the magnetic part of $\phi_{a b c d}$, respectively, where $\phi_{a b c d}^{+}=\tau_{a}{ }^{a^{\prime}} \tau_{b}{ }^{b^{\prime}} \tau_{c}{ }^{c^{\prime}} \tau_{d}{ }^{d^{\prime}} \bar{\phi}_{a b c d}$.

Finally, we get from (5.15)

$$
0=\tau_{d}^{d^{\prime}} \nabla_{d^{\prime}}^{f} \phi_{a b c f}=P_{a b c d}-\frac{3}{4} \epsilon_{d(c} C_{a b)}
$$

with

$$
P_{a b c d}=-\frac{1}{2}\left(P \phi_{a b c d}-2 \mathcal{D}_{(d} f_{\phi_{a b c) f}}\right), \quad C_{a b}=\mathcal{D}^{e f} \phi_{a b e f} .
$$

From this we deduce the system

$$
0=-2 P_{a b c d}=P \phi_{a b c d}-2 \mathcal{D}_{(d}{ }^{f} \phi_{a b c) f}
$$

and the system

$$
\begin{aligned}
& -2 P_{0000}=0, \quad-2 P_{0001}-\frac{1}{2} C_{00}=0, \quad-2 P_{0011}=0 . \\
& -2 P_{0111}+\frac{1}{2} C_{11}=0, \quad-2 P_{1111}=0 .
\end{aligned}
$$

Both these systems are of the form

$$
\sqrt{2} E \partial_{\tau} \phi+A^{a b} c_{a b}^{\mu} \partial_{\mu} \phi=B(\Gamma) \phi .
$$

where the unknown $\phi$ is a "column vector" with complex-valued components

$$
\phi_{p}=\phi_{(a b c d)_{p},}, \quad p=0,1,2,3,4
$$

and the $A^{a b}$ are constant matrices, $B(\Gamma)$ denotes a linear matrix-valued function of the connection cocfficients, and $E$ denotes the $5 \times 5$ unit matrix.

Similarly the constraint equations $C_{a b}=0$ can be written in the form

$$
F^{a b} c_{a b}^{\mu} \partial_{\mu} \phi-H(\Gamma) \phi=0
$$

with constant matrices $F^{a b}$ and a linear matrix-valued function $H(\Gamma)$ of the connection coefficients.

Eqs. (5.46) or (5.47) yield together with (5.39)-(5.45) after a simple transformation (cf. [12]) a symmetric hyperbolic system of propagation equations, the "standard system" in the first and the "boundary adapted system" in the second case.

For convenience we write out the boundary-adapted propagation equations (5.47).

$$
\begin{aligned}
& \left(\sqrt{2}-2 c^{0}{ }_{01}\right) \partial_{\tau} \phi_{0}+2 c^{0}{ }_{00} \partial_{\tau} \phi_{1}-2 c^{\alpha}{ }_{01} \partial_{\alpha} \phi_{0}+2 c^{\alpha}{ }_{00} \partial_{\alpha} \phi_{1} \\
& \quad=\left(2 \Gamma_{0011}-8 \Gamma_{1010}\right) \phi_{0}+\left(4 \Gamma_{0001}+8 \Gamma_{1000}\right) \phi_{1}-6 \Gamma_{0000} \phi_{2} \\
& \sqrt{2} \partial_{\tau} \phi_{1}-c^{0}{ }_{11} \partial_{\tau} \phi_{0}+c^{0}{ }_{00} \partial_{\tau} \phi_{2}-c^{\alpha}{ }_{11} \partial_{\alpha} \phi_{0}+c^{\alpha}{ }_{00} \partial_{\alpha} \phi_{2}
\end{aligned}
$$




$$
\begin{aligned}
&=-\left(4 \Gamma_{1110}+f_{11}\right) \phi_{0}+\left(2 \Gamma_{0011}+4 \Gamma_{1100}-2 f_{01}\right) \phi_{1} \\
&+3 f_{00} \phi_{2}-2 \Gamma_{0000} \phi_{3}, \\
& \sqrt{2} \partial_{\tau} \phi_{2}-c^{0}{ }_{11} \partial_{\tau} \phi_{1}+c^{0}{ }_{00} \partial_{\tau} \phi_{3}-c^{\alpha}{ }_{11} \partial_{\alpha} \phi_{1}+c_{00}^{\alpha} \partial_{\alpha} \phi_{3} \\
&=- \Gamma_{1111} \phi_{0}-2\left(\Gamma_{1101}+f_{11}\right) \phi_{1}+3\left(\Gamma_{0011}+\Gamma_{1100}\right) \phi_{2} \\
&-2\left(\Gamma_{0001}-f_{00}\right) \phi_{3}-\Gamma_{0000} \phi_{4}, \\
& \sqrt{2} \partial_{\tau} \phi_{3}-c_{11}^{0} \partial_{\tau} \phi_{2}+c_{00}^{0} \partial_{\tau} \phi_{4}-c^{\alpha}{ }_{11} \partial_{\alpha} \phi_{2}+c^{\alpha}{ }_{00} \partial_{\alpha} \phi_{4} \\
&=-2 \Gamma_{1111} \phi_{1}-3 f_{11} \phi_{2}+\left(2 \Gamma_{1100}+4 \Gamma_{0011}+2 f_{01}\right) \phi_{3} \\
& \quad-\left(4 \Gamma_{0001}-f_{00}\right) \phi_{4}, \\
&\left(\sqrt{2}+2 c_{01}^{0}\right) \partial_{\tau} \phi_{4}-2 c^{0}{ }_{11} \partial_{\tau} \phi_{3}+2 c^{\alpha}{ }_{01} \partial_{\alpha} \phi_{4}-2 c^{\alpha}{ }_{11} \partial_{\alpha} \phi_{3} \\
&=-6 \Gamma_{1111} \phi_{2}+\left(4 \Gamma_{1110}+8 \Gamma_{0111}\right) \phi_{3}+\left(2 \Gamma_{1100}-8 \Gamma_{0101}\right) \phi_{4} .
\end{aligned}
$$

Constraints (5.49) read explicitly

$$
\begin{aligned}
& c^{0}{ }_{11} \partial_{\tau} \phi_{0}-2 c^{0}{ }_{01} \partial_{\tau} \phi_{1}+c^{0}{ }_{00} \partial_{\tau} \phi_{2}+c^{\alpha}{ }_{11} \partial_{\alpha} \phi_{0}-2 c^{\alpha}{ }_{01} \partial_{\alpha} \phi_{1}+c^{\alpha}{ }_{00} \partial_{\alpha} \phi_{2} \\
&=-\left(2 \Gamma_{(01) 11}-4 \Gamma_{1110}\right) \phi_{0}+\left(2 \Gamma_{0011}-4 \Gamma_{(01) 01}-4 \Gamma_{1100}\right) \phi_{1} \\
&+6 \Gamma_{(01) 00} \phi_{2}-2 \Gamma_{0000} \phi_{3}, \\
& c^{0}{ }_{11} \partial_{\tau} \phi_{1}-2 c^{0}{ }_{01} \partial_{\tau} \phi_{2}+c^{0}{ }_{00} \partial_{\tau} \phi_{3}+c^{\alpha}{ }_{11} \partial_{\alpha} \phi_{1}-2 c^{\alpha}{ }_{01} \partial_{\alpha} \phi_{2}+c^{\alpha}{ }_{00} \partial_{\alpha} \phi_{3} \\
&= \Gamma_{1111} \phi_{0}-\left(4 \Gamma_{(01) 11}-2 \Gamma_{1101}\right) \phi_{1}+3\left(\Gamma_{0011}-\Gamma_{1100}\right) \phi_{2} \\
& \quad-\left(2 \Gamma_{0001}-4 \Gamma_{(01) 00}\right) \phi_{3}-\Gamma_{0000} \phi_{4}, \\
& c^{0}{ }_{11} \partial_{\tau} \phi_{2}-2 c_{01}^{0} \partial_{\tau} \phi_{3}+c^{0}{ }_{00} \partial_{\tau} \phi_{4}+c^{\alpha}{ }_{11} \partial_{\alpha} \phi_{2}-2 c^{\alpha}{ }_{01} \partial_{\alpha} \phi_{3}+c^{\alpha}{ }_{00} \partial_{\alpha} \phi_{4} \\
&= 2 \Gamma_{1111} \phi_{1}-6 \Gamma_{(01) 11} \phi_{2}+\left(4 \Gamma_{0011}+4 \Gamma_{(01) 01}-2 \Gamma_{1100}\right) \phi_{3} \\
&-\left(4 \Gamma_{0001}-2 \Gamma_{(01) 00}\right) \phi_{4} .
\end{aligned}
$$

\subsubsection{The initial data}

Observing our gauge conditions we find for the data on $C_{a, k}^{+}$the following representation. Consider the functions

$$
\check{r}_{a b}^{+}, \check{c}_{a b}^{-}, \quad \check{\gamma}_{a b c d}, \quad s_{a b c d}, \quad r, \quad D_{(a b} D_{c d)} \Omega,
$$

as introduced on $C_{a}^{+}$in Section 3. We use the diffeomorphism $\Sigma$ to transport these functions to $C_{a, k}^{+}$and denote the functions so ubtained by the same symbols. Then the data for the curvature fields are given on $C_{a, \kappa}^{+}$by

$$
\begin{aligned}
& \Theta_{a b c d}=-\frac{\kappa^{2}}{\Omega} D_{(a b} D_{c d)} \Omega+\kappa^{2} \frac{1}{12} r h_{a b c d}, \\
& \phi_{a b c d}=\frac{\kappa^{3}}{\Omega^{2}}\left(D_{(a b} D_{c d)} \Omega+\Omega s_{a b c d}\right) .
\end{aligned}
$$

The data for the frame coefficients are given on $C_{a, k}^{+}$by 


$$
\begin{aligned}
& c_{a b}^{0}=0, \quad c_{a b}^{1}=\kappa x_{a b}, \quad c_{a b}^{+}=\kappa\left(\frac{1}{\rho} z_{a b}+\check{c}_{a b}^{+}\right), \\
& c^{-}{ }_{a b}=\kappa\left(\frac{1}{\rho} y_{a b}+\check{c}_{a b}^{-}\right),
\end{aligned}
$$

where we use $x_{a b}, z_{a b}, y_{a b}$ as given by (3.14). With this notation we write for a given smooth function $\mu$ on $C_{a, \kappa}^{+}$

$$
\mu_{a b}=\kappa^{-1}\left(c^{1} a b \partial_{\rho}+c^{+}{ }_{a b} X_{+}+c^{-} a b X_{-}\right) \mu .
$$

The data for the connection coefficients are then given on $C_{a, k}^{+}$by

$$
\begin{aligned}
& \xi_{a b c d}=\sqrt{2}\left\{\kappa\left(\frac{1}{\rho} \gamma_{a b c d}^{*}+\check{\gamma}_{a b c d}\right)-\frac{1}{2}\left(\epsilon_{a c} \kappa_{b d}+\epsilon_{b d} \kappa_{a c}\right)\right\} . \\
& f_{a b}=\kappa_{a b} . \quad \chi(a b) c d=0 .
\end{aligned}
$$

For later use we note that in the particular case where $\kappa$ is of the form $\kappa=\rho \kappa^{\prime}$ with some smooth function $\kappa^{\prime}$, we get

$$
\xi_{a b c d}=\sqrt{2}\left\{\rho \kappa^{\prime} \check{\gamma}_{a b c d}-\frac{1}{2} \rho\left(\epsilon_{a c} \kappa_{b d}^{\prime}+\epsilon_{b d} \kappa_{a c}^{\prime}\right)\right\} .
$$

In the following we shall always assume the data to be given in cn-gauge.

\subsubsection{Choice of $\kappa$}

Depending on the choice of $\kappa$, we may arrive at quite different initial value problems. The simplest choice is $\kappa=1$ on $\tilde{S}$. In this case scri ${ }^{ \pm}$, if smooth, would be given by

$$
\operatorname{scri}^{ \pm}=\left\{\rho>0, \tau= \pm \omega_{*}\right\}
$$

near space-like infinity. Since $\omega=\mathrm{O}(\rho)$ as $\rho \rightarrow 0$, this choice corresponds to the conformal representations near space-like infinity where space-like infinity is envisioned as a point $i^{0}$. In fact, if we choose $h$ to be flat, which implies $U=1$, and set $W=0$. we arrive at the standard conformal representation of Minkoski space near space-like intinity discussed at the end of Section 6. The basic problem with this choice of $\kappa$ is that the data for $\phi_{a b c d}$ blow up as $\rho \rightarrow 0$.

To avoid that problem we choose a positive analytic function $\kappa^{\prime}$ on $B_{a}(i)$ with $\kappa^{\prime}(i)=1$ (this normalization is somewhat arbitrary), set $\kappa=\rho \kappa^{\prime}$, and consider the analytic lift of $\kappa$ to $C_{a}$.

We can assume $C_{a, k}^{+}$to be extended to an analytic 4-manifold $C_{a, k}$ such that the analytic diffeomorphism $\Sigma$ from $C_{a}^{+}$onto $C_{a, k}^{+}$extends to an analytic diffeomorphism, denoted again by $\Sigma$, from $C_{a}$ onto $C_{a, \kappa}$. The map $\Sigma$ is used to carry the functions $\kappa, T_{m}{ }_{k}, \rho$, $t$, and the vector fields $\partial_{\rho}, X, X_{+}, X_{-}$defined on $C_{a}$ to $C_{a, \kappa}$. The resulting fields will be denoted again by same symbols. Using our function $\kappa$ in (5.57)-(5.56), we find that all data extend analytically through the subset $I^{0}=\{\rho=0\}$ of $C_{a . \kappa}$. Furthermore, we find that the functions $b_{a b}$ as well as the functions $\Omega / \kappa, \kappa^{2} / \omega^{2}, \kappa \Omega / \omega^{2}$, which appear in the expressions for $\Theta, \partial_{\tau} \Theta$, extend in an analytic way through $I^{0}$ with 


$$
\frac{\kappa^{2}}{\omega^{2}} \rightarrow 1 \text { on } I^{0} \text {. }
$$

We assume $a$ to be chosen small enough such that the functions above extend analytically to all of $C_{a, \kappa}$. We shall use the same notation as above for the extended functions.

It follows that our initial value problem can be extended in a regular way, in fact as an initial value problem for a symmetric hyperbolic system, to a larger domain. We introduce the sets

$$
\begin{aligned}
& M_{a}=\left\{(\tau, q) \mid q \in C_{a, \kappa},-\frac{\omega(q)}{\kappa(q)} \leq \tau \leq \frac{\omega(q)}{\kappa(q)}\right\}, \\
& M_{a}^{+}=\left\{(\tau, q) \in M_{a} \mid q \in C_{a, \kappa}^{+}\right\}, \\
& I=\left\{(\tau, q) \in M_{a}\left|q \in I^{0},\right| \tau \mid<1\right\}, \\
& I^{ \pm}=\left\{(\tau, q) \in M_{a} \mid q \in I^{0}, \tau= \pm 1\right\}, \\
& \mathcal{I}_{a}^{ \pm}=\left\{(\tau, q) \in M_{a}^{+} \mid q \in C_{a, \kappa}^{+}, \tau= \pm \frac{\omega(q)}{\kappa(q)}\right\} .
\end{aligned}
$$

The functions $\rho, t, T_{m}{ }^{j}{ }_{k}$ are assumed to be extended off $C_{a, k}$ into $M_{a}$ such that for fixed $q \in C_{a, \kappa}$ they are constant on the curves $\tau \rightarrow(\tau, q) \in M_{a}$.

We shall consider now the initial value problem near space-like infinity as the question whether for some $a>0$ and suitably chosen initial data (5.57)-(5.56) there exists a smooth solution to the propagation equations (5.39)-(5.46) (or (5.47)) $M_{a}^{+}$such that for some $a^{\prime}$, $0<a^{\prime}<a$, the set $M_{a^{\prime}}^{+}$is in the closure in $M_{a}$ of the domain of dependence of the set $C_{a, \kappa}^{+}$ with respect to the given solution.

If the existence of such solution can be established, it follows from [12, Lemma 6.1], that the complete set of conformal field equations (5.4)-(5.8) will be satisfied on $M_{a^{\prime}}^{+}$. The sets $\mathcal{I}_{a^{\prime}}^{ \pm}$on which $\Theta$ vanishes will represent part of future, respectively, past null infinity for this solution. For solutions to Einstein's vacuum field equations with vanishing cosmological constant it is well known that null infinity is represented by a null hypersurface. On the other hand, the function $\Theta$ is given here explicitly. Remarkably, as discussed in [12] (cf. the remarks following Lemma 3.2), no contradiction arises from this.

The extension $M_{a}$ of $M_{a}^{+}$has been introduced here for convenience. Since our propagation equations extend to a symmetric hyperbolic system on $M_{a}$ near $C_{a, k}$ and the data are smooth, it follows from known results (cf. [16,24]) that the problem has a unique, smooth solution on some neighbourhood $W \subset M_{a}$ of $C_{a, \kappa}$. It follows immediately that the solution extends smoothly to the boundary $I \cap W$ of $M_{a}^{+} \cap W$ which represents now part of space-like infinity. The discussion in Section 7 will make it clear why the solution in $M_{a}^{+} \cap W$ does not depend on the extension of the data to $C_{a, \kappa}$.

Of course, the basic problem is to show that the solution extends smoothly to a set $\mathcal{I}_{a^{\prime}}^{ \pm}$, $a^{\prime}>0$. With our choice of the function $\kappa$ we find that the differential of $\Theta$ does not vanish on the sets $I$ and $\mathcal{I}_{a^{\prime}}^{ \pm}$which represent space-like and null infinity respectively. It vanishes, however, on the sets $I^{ \pm}$at which null infinity "touches" space-like infinity. This indicates that the sets $I^{ \pm}$will be of particular interest in the further discussion. 
As an important though immediate consequence of the conformal geodesic equations we note that the choice of a different function $\kappa$ on $\tilde{S}$ neither affects the conformal geodesics, considered as point sets, nor the l-form $b_{\mu}$, or the connection $\hat{\nabla}$ along these curves. The parameter $\tau$ as well as the frame $e_{k}$ suffer rescalings which are constant along the conformal geodesic. The 1 -form $f_{\mu}$ is changed by some additive contribution.

From this follows that the function

$$
\Theta^{\prime}=\kappa_{*} \Theta
$$

would have been the conformal factor which we had obtained if we had required that $\Theta^{\prime}=\Omega$ on $\tilde{S}$. The corresponding time function on the conformal geodesics would have been

$$
\tau^{\prime}=\tau \kappa_{*}
$$

We would have had

$$
\Theta^{\prime}=\Omega_{*}\left(1-\frac{\tau^{\prime 2}}{\omega_{*}^{2}}\right)
$$

and therefore also (5.61). We see that by the simple transformations (5.62), (5.63) and related simple transformations of the other fields we can easily proceed from the picture where space-like infinity is represented by the cylinder $I$ to the picture where space-like infinity is represented by a point $i^{0}$.

\section{The Schwarzschild solution near space-like infinity}

Even if the solutions we wish to construct possessed a smooth structure at null infinity, our gauge conditions could turn out to be unsatisfactory. If a non-vanishing ADM-mass induced the conformal geodesics to form caustics before they reach null infinity, the coordinates associated with them would be useless for our purposes. We therefore test our setting for Schwarzschild data.

In standard coordinates the Schwarzschild line element is given by

$$
\mathrm{d} \tilde{s}^{2}=\left(1-\frac{2 m}{\bar{r}}\right)^{2} \mathrm{~d} t^{2}-\left(1-\frac{2 m}{\bar{r}}\right)^{-2} \mathrm{~d} \bar{r}^{2}-\bar{r}^{2} \mathrm{~d} \sigma^{2}
$$

with $\mathrm{d} \sigma^{2}=\mathrm{d} \theta^{2}+\sin ^{2} \theta \mathrm{d} \phi^{2}$ the standard line element on the unit sphere $S^{2}$ in polar coordinates. Rewriting it in terms of the coordinate $r=\frac{1}{2}(\bar{r}-m+\sqrt{\bar{r}(\bar{r}-2 m)})$ where $\bar{r}>2 m$, we obtain the Schwarzschild line element in isotropic coordinates

$$
\mathrm{d} \tilde{s}^{2}=\left(\frac{1-m / 2 r}{1+m / 2 r}\right)^{2} \mathrm{~d} t^{2}-\left(1+\frac{m}{2 r}\right)^{4}\left(\mathrm{~d} r^{2}+r^{2} \mathrm{~d} \sigma^{2}\right)
$$

We express the first fundamental form $\tilde{h}$ and the second fundamental form $\tilde{\chi}$ on the initial hypersurface $\tilde{S}=\{t=0\}$ in terms of the coordinate $\rho=1 / r$ and get $\tilde{h}=\Omega^{-2} h, \tilde{\chi}=0$ 
with $h=-\left(\mathrm{d} \rho^{2}+\rho^{2} \mathrm{~d} \sigma^{2}\right), \Omega=\rho^{2} /(1+m \rho / 2)^{2}$. Thus the Schwarzschild solution implies data of the type considered in Section 1 with $h$ the flat metric. It follows that $U=1$ near $\rho=0$ and $W=\frac{1}{2} m$.

We set now $\kappa=\rho$ and assume that $a$ is chosen small enough such that $(1+(m / 2) \rho) \neq 0$ for $|\rho|<a$.

The functions entering the evolution equations are given by

$$
\begin{aligned}
& \Theta=\frac{\rho}{(1+\rho m / 2)^{2}}\left(1-\frac{\tau^{2}}{(1+\rho m / 2)^{2}}\right), \\
& \partial_{\tau} \Theta=-2 \tau \frac{\rho}{(1+\rho m / 2)^{4}}, \quad d_{a b}=\frac{2 \rho x_{a b}}{(1+\rho m / 2)^{3}},
\end{aligned}
$$

and the initial data on $C_{a . k}$ are given by

$$
\begin{aligned}
& c_{a b}^{0}=0, \quad c_{a b}^{1}=\rho x_{a b}, \quad c_{a b}^{-}=y_{a b}, \quad c^{+}{ }_{a b}=z_{a b} . \\
& f_{a b}=x_{a b}, \quad \xi_{a b c d}=0, \quad \chi_{(a b) c d}=0, \\
& \Theta_{a b c d}=\frac{6 m \rho}{(1+\rho m / 2)^{2}} \epsilon_{a b c d}^{2}, \quad \phi_{a b c d}=-6 m \epsilon^{2}{ }_{a b c d} .
\end{aligned}
$$

We assume the ansatz

$$
\begin{aligned}
& c_{a b}^{0}=c^{0} x_{a b}, \quad c_{a b}^{1}=c^{1} x_{a b}, \quad c^{-}{ }_{a b}=c^{-} y_{a b}, \quad c^{+}{ }_{a b}=c^{+} z_{a b}, \\
& f_{a b}=f x_{a b}, \quad \xi_{a b c d}=\frac{1}{\sqrt{2}} \xi\left(\epsilon_{a c} x_{b d}+\epsilon_{b d} x_{a c}\right), \\
& \chi_{(a b) c d}=\chi_{4} \epsilon^{2}{ }_{a b c d}+\frac{1}{3} \chi h_{a b c d}, \\
& \Theta_{a b c d}=\Theta_{4} \epsilon^{2}{ }_{a b c d}+\frac{1}{3} \Theta_{T} h_{a b c d}+\frac{1}{\sqrt{2}} \Theta_{R} \epsilon_{a b} x_{c d}, \quad \phi_{a b c d}=\phi \epsilon^{2}{ }_{a b c d}
\end{aligned}
$$

for the solution of the propagation equations. Here the components of the unknown

$$
u=\left(c^{0}, c^{1}, c^{-}, c^{+}, f, \xi, \chi_{4}, \chi, \Theta_{4}, \Theta_{T}, \Theta_{R}, \phi\right)
$$

are real-valued functions of $\tau$ and $\rho$. The initial data $u_{0}=\left.u\right|_{\tau=0}$ are given by

$$
\begin{aligned}
& c^{0}=0, \quad c^{1}=\rho, \quad c^{-}=c^{+}=1, \quad f=1, \quad \xi=0, \quad \chi_{4}=0, \quad \chi=0, \\
& \Theta_{4}=\frac{6 m \rho}{(1+\rho m / 2)^{2}}, \quad \Theta_{T}=0, \quad \Theta_{R}=0, \quad \phi=-6 m .
\end{aligned}
$$

The propagation equations (5.39)-(5.46) reduce to

$$
\begin{aligned}
\partial_{\tau} c^{0} & =\frac{1}{3}\left(\chi_{4}-\chi\right) c^{0}-f, & & \partial_{\tau} c^{1}=\frac{1}{3}\left(\chi_{4}-\chi\right) c^{1}, \\
\partial_{\tau} c^{ \pm} & =-\frac{1}{6}\left(\chi_{4}+2 \chi\right) c^{ \pm}, & & \partial_{\tau} \xi=-\frac{1}{6}\left(\chi_{4}+2 \chi\right) \xi-\frac{1}{2} \chi_{4} f-\Theta_{R}, \\
\partial_{\tau} f & =\frac{1}{3}\left(\chi_{4}-\chi\right) f+\Theta_{R}, & & \partial_{\tau} \chi_{4}=\frac{1}{6} \chi_{4}^{2}-\frac{2}{3} \chi_{4} \chi-\Theta_{4}+\Theta \phi, \\
\partial_{\tau} \chi & ={ }_{6}^{1} \chi_{4}^{2} \quad \frac{1}{3} \chi^{2} \quad \Theta_{T}, & & \partial_{\tau} \Theta_{4}=\frac{1}{6} \chi_{4} \Theta_{4}-\frac{1}{3}\left(\chi_{4} \Theta_{T}+\chi \Theta_{4}\right)-\partial_{\tau} \Theta \phi, \\
\partial_{\tau} \Theta_{T} & =-\frac{1}{6} \chi_{4} \Theta_{4}-\frac{1}{3} \chi \Theta_{T}, & & \partial_{\tau} \Theta_{R}=\frac{1}{3}\left(\chi_{4}-\chi\right) \Theta_{R}-\frac{2 \rho}{3(1+\rho m / 2)^{3}} \phi, \\
\partial_{\tau} \phi & =-\frac{1}{2}\left(\chi_{4}+2 \chi\right) \phi . & &
\end{aligned}
$$


Thus the problem of reconstructing the conformal Schwarzschild solution from the given data amounts to finding a solution $u=u(\tau, \rho ; m)$ of an initial value problem of the type

$$
\partial_{\tau} u=F(u, \tau, \rho ; m), \quad u(0, \rho ; m)=u_{0}(\rho ; m)
$$

with analytic functions $F$ and $u_{0}$. For given values $\rho, m$ with $1+\rho m / 2>0$, there exists a smooth solution close to $\tau=0$. It represents part of the conformal Schwarzschild solution.

For $m=0$ the only non-vanishing components of the solution $u$ are given for $\tau, \rho \in \mathbb{R}$ by

$$
c^{0}=-\tau, \quad c^{1}=\rho, \quad c^{ \pm}=1, \quad f=1,
$$

which entails

$$
\tau_{a a^{\prime}} \sigma^{a a^{\prime}}=\sqrt{2}\left(\mathrm{~d} \tau+\frac{\tau}{\rho} \mathrm{d} \rho\right) . \quad \sigma^{a b}=-\frac{1}{\rho} x^{a b} \mathrm{~d} \rho-2 y^{a b} \alpha^{+}-2 z^{a b} \alpha^{-} .
$$

The pull back under the Hopf map of the standard metric on the 2-sphere is given by $2\left(\alpha^{-} \alpha^{+}+\alpha^{+} \alpha^{-}\right)$. We denote it again by $\mathrm{d} \sigma^{2}$. Observing (5.35) we obtain

$$
g=\frac{1}{\rho^{2}}\left\{\mathrm{~d}(\rho \tau)^{2}-\mathrm{d} \rho^{2}-\rho^{2} \mathrm{~d} \sigma^{2}\right\}
$$

Notice that $g\left(\partial_{\rho}, \partial_{\rho}\right) \rightarrow \infty$ as $\rho \rightarrow 0,|\tau|<1$. A coordinate better adapted to the metric (6.4) for small values of $\rho$ would be $r=-\log (\rho)$ which leads to

$$
g=\mathrm{d} \tau^{2}-2 \tau \mathrm{d} \tau \mathrm{d} r-\left(1-\tau^{2}\right) \mathrm{d} r^{2}-\mathrm{d} \sigma^{2} .
$$

It is seen that the cylinder $I$ lies at infinity but has a finite circumference with respect to the metric $g$. The null hypersurfaces scri ${ }^{ \pm}=\{\tau= \pm 1\}$ have a finite location.

In the conformally flat case we find that $f_{a b} \sigma^{a b}=(1 / \rho) \mathrm{d} \rho$ is closed and the connection $\hat{\nabla}$ is in fact the Levi-Civita connection of the metric $\rho^{2} g$. Writing $x^{0}=\tau \rho$, we get from (6.4)

$$
\tilde{g}=\Theta^{-1} g=\frac{1}{\left(\rho^{2}-\left(x^{0}\right)^{2}\right)^{2}}\left\{\left(\mathrm{~d} x^{0}\right)^{2}-\mathrm{d} \rho^{2}-\rho^{2} \mathrm{~d} \sigma^{2}\right\} .
$$

Since this metric is invariant under the action of the group $U(1)$ it descends to the underlying manifold. Assuming $\rho$ to be the radial coordinate associated with spatial normal coordinates $x^{a}, a=1,2,3$, we get the expression

$$
\tilde{g}=\frac{1}{\left(x_{\lambda} x^{\lambda}\right)^{2}} \eta_{\mu \nu} \mathrm{d} x^{\mu} \mathrm{d} x^{\prime \prime}
$$

which after the inversion $x^{\mu} \rightarrow-x^{\mu} / x_{\lambda} x^{\lambda}$ gives near space-like infinity the standard representation of the Minkowski metric.

Since for $m=0$ the solution exists for $\tau, \rho \in \mathbb{R}$, it follows from well known results on ordinary differential equations, that for a given value $\tau_{0}>1$ there exist numbers $m_{0}>0$, $\rho_{0}>0$ such that the solution $u(\tau, \rho ; m)$, which is analytic in all variables, exists for $|\tau| \leq \tau_{0},|\rho| \leq \rho_{0},|m| \leq m_{0}$. It is defined in particular on the sets $\{\tau= \pm(1+\rho m / 2), 0<$ 
$\left.\rho<\min \left(\rho_{0},(2 / m)\left(\tau_{0}-1\right)\right)\right\}$ which represent part of null infinity of the corresponding Schwarzschild space-time. With the exception of $\phi$, the initial data contain $\rho$ and $m$ only in form of the product $\rho m$ and in the equation only such products or the product $\rho \phi$ occur. This implies for arbitrary $m>0$ the existence of solutions which extend smoothly through null infinity for sufficiently small values of $\rho$.

Thus we have obtained a system of coordinates near space-like infinity which is simultaneously analytic through future and past null infinity. No caustics of the underlying congruence of conformal geodesics occur before the latter have passed null infinity. The coordinates should allow to analyse the behaviour of fields near space-like infinity to any desired degree of precision. It is remarkable that in the coordinates above conformal Minkowski spacetime is obtained in a fixed coordinate neighbourhood of $\bar{I}=\{|\tau| \leq 1, \rho=0\}$ as a uniform limit of Schwarzschild solutions as $m \rightarrow 0$.

In our conformal representation (6.4) of Minkowski space the null geodesics orthogonal to the spheres $\{\rho=$ const. $>0\}$ are given by

$$
\tau=\frac{s}{1 \mp s}, \quad \rho=\rho_{0}(1 \mp s), \quad t^{a}{ }_{b}=\text { const. }
$$

The outgoing geodesics, for which the minus sign applies, arrive at $\mathrm{scri}^{+}$for the value $s=\frac{1}{2}$ at which $\rho=\frac{1}{2} \rho_{0}$. It follows from the remark above that corresponding outgoing null geodesics of the conformal Schwarzschild solution behave more and more like those for the Minkowski solution as $\rho_{0} \rightarrow 0$ or $m \rightarrow 0$. The incoming geodesics do not arrive at $\mathrm{scri}^{+}$for a finite value of $s$. As $\rho_{0} \rightarrow 0$, these curves approach the set $\{0 \leq \tau \leq 1, \rho=$ $0\} \cup\{\tau=1, \rho>0\}$ in a non-uniform way. The corresponding behaviour in the case of the conformal Schwarzschild solution together with the detailed structure of this solution near $I^{+}$should allow one to exhibit the source of the non-smoothness of the family of incoming null hypersurfaces at scri ${ }^{+}$.

\section{The characteristic $I$ at space-like infinity}

We consider the system (5.39)-(5.45) and (5.48) in $M_{a}$ with data given on $C_{a}$. For definiteness we assume the boundary adapted system (5.47). The complete system is of the form

$$
\left\{A^{\prime 0} \partial_{\tau}+A^{\prime 1} \partial_{\rho}+A^{\prime+} X_{+}+A^{\prime-} X_{-}\right\} u=C^{\prime} u
$$

with a "vector"-valued unknown

$$
u=\left(c_{a b}^{0}, c_{a b}^{\alpha}, \Gamma_{a b c d}, \Theta_{a b c d}, \phi_{a b c d}\right) .
$$

Here $\alpha=1, \pm$, and $A^{\prime 0}, A^{\prime}, A^{\prime \pm}, C^{\prime}$ denote matrix-valued functions which depend on $u$ and the coordinates. We consider now a neighbourhood $W$ of $C_{a, k}$ in $M_{a}$ on which a unique smooth (analytic) solution of our Cauchy problem is given and analyse the behaviour of the solution near the set $I^{\prime}=I \cap W$ which we assume to be diffeomorphic to $[c, c] \times S U(2, C)$ with some $c>0$. 
We have seen that from the point of view of the metric $g$ the set $I^{\prime}$ arises in a singular representation of the metric. From the point of view of the field equations (5.39)-(5.45), (5.48) the set $I^{\prime}$ is a regular hypersurface. We write $u^{0}=\left.u\right|_{I^{\prime}}$ and use a similar notation for other functions. Since the function $\Theta$ vanishes on $I^{\prime}$, Eqs. (5.39)-(5.45) decouple on $I^{\prime}$ from (5.48) and can be integrated on $I^{\prime}$. Since the restrictions of the initial data (5.57)-(5.55) to $I^{0}$ coincide, irrespective of the choice of the function $\kappa^{\prime}$, with the restriction of Minkowski data, we find on $I^{\prime}$

$$
\begin{aligned}
& \Theta_{a b c d}^{0}=0, \quad \chi_{(a b) c d}^{0}=0, \quad f_{a b}^{0}=x_{a b}, \quad \xi_{a b c d}^{0}=0, \\
& \left(c_{a b}^{0}\right)^{0}=-\tau x_{a b}, \quad\left(c^{1}{ }_{a b}\right)^{0}=0, \quad\left(c^{-}{ }_{a b}\right)^{0}=y_{a b}, \quad\left(c^{+}{ }_{a b}\right)^{0}=z_{a b} .
\end{aligned}
$$

It follows from this that

$$
A^{\prime}=0 \text { on } I^{\prime}
$$

and in particular that the system (5.48) also implies an interior system on $I^{\prime}$. Observing (5.56), the integration gives

$$
\phi_{a b c d}^{0}=-6 m \epsilon^{2} \text { abcd } .
$$

We see that $I^{\prime}$ is a characteristic of the system (5.39)-(5.45), (5.48). It is a "total characteristic" in the sense that the complete system reduces to a (symmetric hyperbolic) system of interior cquations on $I^{\prime}$. Also the constraints (5.49) induce interior equations on $I^{\prime}$. By repeated application of $\partial_{\rho}$ to Eqs. (5.39)-(5.45) and (5.48) we obtain symmetric hyperbolic interior systems for the quantities $\left.u^{p} \equiv \partial_{\rho}^{p} u\right|_{I^{\prime}}, p=0,1,2 \ldots$ where the coefficients and right-hand sides in the system for $u^{p}, p \geq 1$, are given in terms the quantities $u^{q}$. $q=0.1, \ldots, p-1$. Obviously, these systems can be deduced in a formal way and studied on the whole set $I$.

Another important observation is the following. If the solution extends smoothly to the sets $\mathcal{I}_{a}^{ \pm}$. the latter will necessarily be null hypersurfaces whence characteristics for the propagation equations. Since they extend transversely across $I \cup I^{+} \cup I^{-}$and $I$ is a total characteristic, there should occur a degeneracy of the propagation equations on the sets $I^{ \pm}$. It follows in fact from (7.3) that we have in (5.48)

$$
A^{0} \equiv \sqrt{2} E+A^{a b} c^{0}{ }_{a b}=\sqrt{2} \operatorname{diag}\{1+\tau, 1,1,1,1-\tau\} \quad \text { on } I .
$$

Thus the matrix $A^{0}$, which is positive definite on $I$, degenerates on the sets $I^{ \pm}$. This degeneracy represents the essential remaining problem in our discussion. If the matrix $A^{0}$ were positive definite on $I$, we could deduce the existence of solutions with a smooth asymptotic structure near spatial infinity from well-known stability results for solutions of symmetric hyperbolic systems.

To see the effect of the degeneracy we shall analyse the behaviour of the functions $u^{p}$ on $I$. To simplify the calculations we shall make the specific choice

$$
\kappa^{\prime}=1 \text {. }
$$


It should be observed that under certain circumstances this choice might have disadvantages. In our study of Minkowski space this choice resulted in the relation $\omega_{*} / \kappa_{*}=1$. This entails that $\tau=$ const. on the characteristic hypersurfaces $\mathcal{I}_{a}^{ \pm}$. As a consequence the matrix $A^{0}$ degenerates on $\mathcal{I}_{a}^{ \pm}$. It should be observed that this degeneracy can be removed while the degeneracy at $I^{ \pm}$cannot be removed by a suitable choice of $\kappa^{\prime}$.

Expanding all quantities in terms of the $T_{m}{ }^{j}{ }_{k}$, the functions $u^{p}$ are obtained on $I$ as solutions to ordinary differential equations. By direct integration we obtain

$$
\begin{aligned}
\Theta_{a b c d}^{1}= & 6 m\left(1-\tau^{2}\right) \epsilon_{a b c d}^{2}+2 \sqrt{2} m \tau \epsilon_{a b} x_{c d}, \\
\chi_{(a b) c d}^{1}= & m\left(-12 \tau+4 \tau^{3}\right) \epsilon_{a b c d}^{2}, \quad f_{a b}^{1}=m \frac{1}{3} \tau^{4} x_{a b}, \\
\xi_{a b c d}^{1}= & \sqrt{2} m\left(\frac{1}{2} \tau^{2}-\frac{1}{4} \tau^{4}\right)\left(\epsilon_{a c} x_{b d}+\epsilon_{b d} x_{a c}\right), \\
\left(c^{-}{ }_{a b}\right)^{1}= & m\left(\tau^{2}-\frac{1}{6} \tau^{4}\right) y_{a b}, \quad\left(c^{+} a b\right)^{1}=m\left(\tau^{2}-\frac{1}{6} \tau^{4}\right) z_{a b}, \\
\left(c_{a b}^{1}\right)^{1}= & x_{a b}, \quad\left(c^{0}{ }_{a b}\right)^{1}=m\left(\frac{4}{3} \tau^{3}-\frac{1}{3} \tau^{5}\right) x_{a b}, \\
\phi_{a b c d}^{1}= & -\left\{W_{1} 36\left(1-\tau^{2}\right)+m^{2}\left(18 \tau^{2}-3 \tau^{4}\right\} \epsilon_{a b c d}^{2}\right. \\
& -12(1-\tau)^{2} X_{+} W_{1} \epsilon_{a b c d}^{1}+12(1+\tau)^{2} X_{-} W_{1} \epsilon_{a b c d}^{3} .
\end{aligned}
$$

Because $\phi_{(a b c d)_{j}}^{1}=0$ for $j=0,4$, no problem arises at this stage from the degeneracy of $A^{\tau}$ on $I^{ \pm}$.

Since $\Theta_{f}{ }_{a b} \neq 0$ if $m \neq 0$ by (7.7), we see that the Ricci tensor of $\hat{\nabla}$ is non-symmetric and therefore $\hat{\nabla}$ is no longer metric if $m \neq 0$.

In the following we shall use the expressions for the initial data obtained in Section 3.2 in the cn-gauge. A lengthy but straightforward calculation gives the following expansion coefficients.

$$
\begin{aligned}
\Theta_{f}^{2 f} f_{a b}= & \sqrt{2}\left\{m^{2}\left(-12 \tau-\frac{8}{3} \tau^{3}+\frac{4}{3} \tau^{5}\right)+W_{1}\left(48 \tau-16 \tau^{3}\right)\right\} x_{a b} \\
& +\sqrt{2}\left(24 \tau+8 \tau^{3}\right)\left(X_{-} W_{1} y_{a b}+X_{+} W_{1} z_{a b}\right) \\
\Theta_{(a b) c d}^{2}= & \left\{m^{2}\left(-12+12 \tau^{2}-10 \tau^{4}+\frac{2}{3} \tau^{6}\right)+W_{1}\left(72-72 \tau^{2}+36 \tau^{4}\right)\right\} \epsilon^{2} a b c d \\
& +m^{2}\left(4 \tau^{2}-\frac{8}{3} \tau^{4}+\frac{4}{9} \tau^{6}\right) h_{a b c d}+24 \tau^{2}\left(X_{-} W_{1} y_{a b}+X_{+} W_{1} z_{a b}\right) x_{c d} \\
& +\left(24-24 \tau^{2}-12 \tau^{4}\right)\left\{X_{+} W_{1} \epsilon^{1}{ }_{a b c d}-X_{-} W_{1} \epsilon^{3}{ }_{a b c d}\right\} \\
\chi_{(a b) c d}^{2}= & \left\{m^{2}\left(24 \tau-8 \tau^{3}+4 \tau^{5}-\frac{4}{21} \tau^{7}\right)\right. \\
& \left.+W_{1}\left(-144 \tau+72 \tau^{3}-\frac{108}{5} \tau^{5}\right)\right\} \epsilon^{2}{ }_{a b c d} \\
& +m^{2}\left(-\frac{20}{3} \tau^{3}+\frac{8}{3} \tau^{5}-\frac{20}{63} \tau^{7}\right) h_{a b c d} \\
& -8 \tau^{3} x_{a b}\left(X-W_{1} y_{c d}+X_{+} W_{1} z_{c d}\right) \\
& +\left(-48 \tau+8 \tau^{3}+\frac{36}{5} \tau^{5}\right)\left(X_{+} W_{1} \epsilon_{a b c d}-X_{-} W_{1} \epsilon_{a b c d}^{3}\right) \\
f_{a b}^{2}= & \left\{m^{2}\left(-2 \tau^{2}+\frac{1}{3} \tau^{4}-\frac{4}{9} \tau^{6}+\frac{1}{7} \tau^{8}\right)+W_{1}\left(2 \tau^{4}-\frac{6}{5} \tau^{6}\right)\right\} x_{a b} \\
& +\left(3 \tau^{4}+\frac{3}{5} \tau^{6}\right)\left(X_{-} W_{1} y_{a b}+X_{+} W_{1} z_{a b}\right) \\
\xi_{a b c d}^{2}= & \sqrt{2}\left\{m^{2}\left(\frac{4}{3} \tau^{4}-\frac{2}{9} \tau^{6}-\frac{1}{28} \tau^{8}\right)+W_{1}\left(6 \tau^{2}-\frac{5}{2} \tau^{4}+\frac{9}{10} \tau^{6}\right)\right\} \\
& \times\left(\epsilon_{a c} x_{b d}+\epsilon_{b d} x_{a c}\right)+\sqrt{2}\left(-\frac{3}{2} \tau^{4}-\frac{3}{10} \tau^{6}\right)
\end{aligned}
$$




$$
\begin{aligned}
& \times\left\{\epsilon_{a c}\left(X_{-} W_{1} y_{b d}+X_{+} W_{1} z_{b d}\right)+\epsilon_{b d}\left(X_{-} W_{1} y_{a c}+X_{+} W_{1} z_{a c}\right)\right\} \\
& +\left(12 \tau^{2}+\tau^{4}-\frac{3}{5} \tau^{6}\right) x_{a b}\left(X_{-} W_{1} y_{c d}-X_{+} W_{1} z_{c d}\right) \\
& +\left(-24 \tau^{2}+12 \tau^{4}\right)\left(X_{+} W_{1} \epsilon_{a b c d}^{1}+X_{-} W_{1} \epsilon_{a b c d}^{3}\right) \\
\left(c^{-}{ }_{a b}\right)^{2}= & \left\{m^{2}\left(-2 \tau^{2}+3 \tau^{4}-\frac{8}{9} \tau^{6}+\frac{1}{14} \tau^{8}\right)+W_{1}\left(12 \tau^{2}-3 \tau^{4}+\frac{3}{5} \tau^{6}\right)\right\} y_{a b} \\
& +\left(-6 \tau^{2}-\frac{1}{2} \tau^{4}+\frac{3}{10} \tau^{6}\right) X_{+} W_{1} x_{a b}, \\
\left(c^{+}{ }_{a b}\right)^{2}= & \left\{m^{2}\left(-2 \tau^{2}+3 \tau^{4}-\frac{8}{9} \tau^{6}+\frac{1}{14} \tau^{8}\right)+W_{1}\left(12 \tau^{2}-3 \tau^{4}+\frac{3}{5} \tau^{6}\right)\right\} z_{a b} \\
& +\left(-6 \tau^{2}-\frac{1}{2} \tau^{4}+\frac{3}{10} \tau^{6}\right) X_{-} W_{1} x_{a b}, \\
\left(c^{1}{ }_{a b}\right)^{2}= & m\left(-4 \tau^{2}+\frac{2}{3} \tau^{4}\right) x_{a b}, \\
\left(c^{0}{ }_{a b}\right)^{2}= & \left\{m^{2}\left(-2 \tau^{3}-3 \tau^{5}+\frac{8}{7} \tau^{7}-\frac{1}{7} \tau^{9}\right)+W_{1}\left(16 \tau^{3}-\frac{26}{5} \tau^{5}+\frac{6}{5} \tau^{7}\right)\right\} x_{a b} \\
& +\left(8 \tau^{3}-\frac{7}{5} \tau^{5}-\frac{3}{5} \tau^{7}\right)\left\{X_{-} W_{1} y_{a b}+X_{+} W_{1} z_{a b}\right\} .
\end{aligned}
$$

The field $\phi_{a b c d}^{2}$ is given on $I$ as a sum of three terms. The solution for vanishing initial data on $I^{0}$ to the inhomogeneous equations satisfied by $\phi_{a b c d}^{2}$ (cf. Eqs. (8.10)-(8.14)) is of the form

$$
\begin{aligned}
& \phi_{(a b c d)_{0}}^{2 i h}=0, \quad \phi_{(a b c d)_{4}}^{2 i h}=0, \quad \phi_{(a b c d)_{1}}^{2 i h}=a_{1}(\tau) m X_{+} W_{1}, \\
& \phi_{(a b c d)_{2}}^{2 i h}=a_{2}(\tau) m W_{1}+c_{2}(\tau) m^{3}, \quad \phi_{(a b c d)_{3}}^{2 i h}=a_{3}(\tau) m X_{-} W_{1},
\end{aligned}
$$

with

$$
\begin{aligned}
& a_{1}(\tau)=-18 \tau^{2}+46 \tau^{3}-\frac{41}{2} \tau^{4}-\frac{41}{5} \tau^{5}+\frac{37}{10} \tau^{6}=-a_{3}(-\tau), \\
& a_{2}=-72 \tau^{2}+62 \tau^{4}-\frac{46}{5} \tau^{6}, \quad c_{2}=6 \tau^{2}-15 \tau^{4}+3 \tau^{6}-\frac{8}{21} \tau^{8} .
\end{aligned}
$$

It extends smoothly to $I^{ \pm}$.

The solution to the homogeneous equation which takes on $I^{0}$ the initial data induced by $\phi_{a b c d}^{W}(\mathrm{cf} .(4.49))$ is given by

$$
\check{\phi}_{(a b c d)_{j}}^{W 2}=-4 \sqrt{6\left(\begin{array}{l}
4 \\
j
\end{array}\right)}(1+\tau)^{j}(1-\tau)^{4-j} \sum_{k=0}^{4} W_{2 ; 4, k} T_{4}{ }^{k}{ }_{j} .
$$

It extends smoothly to $I^{ \pm}$.

The solution to the homogeneous equation which takes on $I^{0}$ the initial data induced by $\phi_{a b c d}^{\prime}(\mathrm{cf} .(4.50))$ is given by

$$
\breve{\phi}_{(a b c d)_{j}}^{2}=a_{j}(\tau) \frac{1}{3} \sum_{k=0}^{4} \sqrt{2\left(\begin{array}{l}
4 \\
k
\end{array}\right)} b_{(a b c d)_{k}}^{*} T_{4}^{k}
$$

with

$$
\begin{aligned}
& a_{0}(\tau)=2(1-\tau)^{4} K(-\tau)=-a_{4}(-\tau), \\
& a_{1}(\tau)=4(1-\tau)^{3}(1+\tau) K(-\tau)-\frac{3}{1-\tau}=-a_{3}(-\tau),
\end{aligned}
$$




$$
a_{2}(\tau)=\sqrt{6}\left\{\frac{2-\tau}{(1+\tau)^{2}}-2(1-\tau)^{2}(1+\tau)^{2} K(\tau)\right\}=-a_{2}(-\tau),
$$

where

$$
K(\tau)=1-3 \int_{0}^{\tau} \frac{\mathrm{d} s}{(1-s)(1+s)^{5}}
$$

This part of $\phi_{a b c d}^{2}$ has logarithmic singularities at $I^{ \pm}$unless $b_{a b c d}=0$ at $i$.

The transport equations on $I$ are invariably connected with the propagation equations. Singularities of solutions to the transport equations will most likely affect the nature of the solution in $M_{a}$. To gain control on the smoothness of the solution near space-like infinity we therefore need to study the behaviour of the solutions to the transport equations on $I$.

In the following section we shall study the occurrence of the type of logarithmic singularity observed above in a systematic way.

\section{The transport equations on $I$}

The role of condition (4.7) is illustrated by the following simple situation.

Theorem 8.1. Suppose that $\phi_{\text {und }}^{W}=0$ near $i$. Then the solution to the initial value problem near space-like infinity extends as an analytic solution to $\mathcal{I}_{a}^{ \pm}$for some a $>0$ if condition (4.7) is satisfied.

This follows from the discussion in [10] and Theorem 4.2. Since by our assumptions the rescaled conformal Weyl tensor is analytic at $i$, we can pose near space-like infinity a regular Cauchy problem for the conformal field equations in the form in which they have been used in [11]. This will give an analytic solution near $i$. The cone generated by the null geodesics through $i$ provides then the sets $\mathcal{I}_{a}^{ \pm}$.

The assumption of analyticity is made here mainly for convenience. If $\phi_{a b c d}^{W}=0$ near $i$ and condition (4.7) is satisfied only to some (sufficiently high) order $p$, we could still show the existence of a solutions near space-like infinity with a differentiable structure at null infinity, the differentiability of the latter depending on $p$. It follows that condition (4.7), possibly only to a certain order, is a necessary regularity condition. The proof and the precise analysis of the differentiability properties require in this case complicated recursion arguments.

The formalism developed here is designed to allow a discussion of the questions of smoothness also in the presence of a non-vanishing massive part of the rescaled Weyl spinor. The analysis of the transport equations on $I$ allows us to recognize again the role of (4.7) as a necessary regularity condition.

Theorem 8.2. The solution to the regular finite initial value problem is smooth through $I^{ \pm}$only if condition (4.7) is satisfied by the free initial data. If (4.7) is violated at some order $q$, the solution will develop logarithmic singularities at $I^{ \pm}$. 
Remarks. It is important to note that our gauge conditions are defined entirely in terms of the conformal structure of the solution. Thus also the logarithmic singularities observed here are associated directly with the conformal structure. They are not due to obscure gauge conditions. Furthermore, it is remarkable that the source of the logarithmic singularities can be identified in the structure of the data.

Our result is a statement about the solution to the initial value problem posed in Section 5 . A priori the smoothness of the solution along $I$ is not our main concern. We could live with a situation where a singularity develops at $I^{ \pm}$which does not affect the smoothness of null infinity. However, most likely any singularity at $I^{ \pm}$will "spread along null infinity".

It is well known that for static solutions having sufficiently fast fall-off behaviour on a slice of time symmetry, null infinity admits a smooth structure. As shown in [10], the data on the slice of time symmetry do satisfy condition (4.2) as a consequence of the static field equations. We expect this to be the structural reason for the smoothness of scri in the case of static solutions.

The results of the following discussion allow us to control more properties of the solutions to the transport equations than those asserted in Theorem 8.2. Apart from the calculational complexities our results will show how to determine explicitly the formal expansion $\sum_{p=0}^{\infty}(1 / p !) u^{p} \rho^{p}$ on $I$ to any given order. Moreover, we consider part (i) of Lemma 8.6 as an indication that condition (4.7) may be the only regularity requirement which needs to be observed. Part (iii) of Lemma 8.6 indicates one of the difficulties to complete the analysis.

Spin weights are preserved by performing analytic lifts of spinor fields and their transport to $C_{a, k}$. Furthermore, they are preserved by the propagation equations. Analytic functions of well-defined spin weights given in some neighbourhood of $I^{n}$ in $M_{a}$ can be expanded in the form (3.39) and (3.42) with coefficients which are analytic functions of $\tau$. To these functions the notion of expansion type introduced in Section 2 extends in an obvious way.

For the functions which enter the initial conditions we find:

Lemma 8.3. Assume, as above, that $\kappa=\rho \kappa^{\prime}$. Then the functions $\Theta, \partial_{\tau}\left(\Theta, d_{a b}\right.$ are of expansion tvpe $p-1$ on $M_{a}$. Assume furthermore that the data are given in cn-gauge. Then the analytic extensions of the functions

$$
(U+\rho W)^{k}-1, \quad \frac{1}{\rho} d_{a b}-2 x_{a b} . \quad\left(\frac{\rho}{\omega}\right)^{2}-1, \quad \frac{\Omega}{\rho^{2}}-1
$$

are of expansion type $p-1$. In particular, if in addition $\kappa^{\prime}=1$, the functions

$$
\frac{\Theta}{\rho}-\left(1-\tau^{2}\right), \quad \frac{\partial_{\tau} \Theta}{\rho}+2 \tau
$$

are of expansion type $p-1$ on $M_{a}$.

This follows from (5.36), (5.37), and Lemma 4.7. For the initial data we get: 
Lemma 8.4. Suppose the initial data are given in cn-gauge and $\kappa^{\prime}=1$. Then the analytic lifts to $C_{a, k}$ of the fields

$$
\begin{aligned}
& c_{a b}^{0}, \quad c_{a b}^{1}, \quad c_{a b}^{+}-z_{a b}, \quad c_{a b}^{-}-y_{a b}, \\
& \chi_{(a b) c d}, \quad f_{a b}-x_{a b}, \quad \xi_{a b c d}, \quad \Gamma_{a b c d}+\frac{1}{2} \epsilon_{a b} x_{c d} \\
& \Theta_{a b c d}=-\frac{\Omega}{\kappa} \phi_{a b c d}+\kappa^{2}\left(s_{a b c d}+\frac{1}{12} r h_{a b c d}\right)
\end{aligned}
$$

are of expansion type $p-1$.

This follows from Lemmas 4.7, 8.3 and Theorem 4.1.

We shall now derive the transport equations on $I$ in general form and make a few general observations about their solutions. Introducing the notation

$$
v=\left(c_{a b}^{0}, c_{a b}^{\alpha}, \Gamma_{a b c d}, \Theta_{a b c d}\right),
$$

we can write Eqs. (5.39)-(5.45) in the form

$$
\partial_{\tau} v=K v+Q(v, v)+L \phi
$$

where $K, Q$ denote linear and quadratic functions respectively, which have constant coefficients, and $L$ denotes a linear function with coefficients which depend on the coordinates such that $L^{0}=0$. We thus get on $I$ transport equations

$$
\begin{aligned}
\partial_{\tau} v^{p}-K & v^{p}+Q\left(v^{0}, v^{p}\right)+Q\left(v^{p}, v^{0}\right) \\
& +\sum_{j=1}^{p-1}\left(\begin{array}{l}
p \\
j
\end{array}\right)\left\{Q\left(v^{j}, v^{p-j}\right)+L^{j} \phi^{p-j}\right\}+L^{p} \phi^{0}, \quad p=1,2,3, \ldots
\end{aligned}
$$

where the values of $v^{0}, \phi^{0}$ given by (7.2), (7.3) and (7.5) are assumed.

By taking formal derivatives of (5.48), restricting to $I$, and observing the values determined above for $v^{0}, v^{1}, \phi^{0}$ we get transport equations

$$
\mathcal{A}_{p} \phi^{p}=R^{p}, \quad p=1,2,3, \ldots
$$

with a linear differential operator

$$
\mathcal{A}_{p} \phi^{p}=\left\{\left(E-\tau A^{01}\right) \partial_{\tau}+(p-2) A^{01}+D+\frac{1}{2}\left(A^{00} X_{+}-A^{11} X_{-}\right)\right\} \phi^{p},
$$

where $D=\operatorname{diag}(0,1,0,-1,0)$, and a "column vector" $R^{p}$ given by

$$
\begin{aligned}
\sqrt{2} R^{p}= & -\sum_{j=2}^{p}\left(\begin{array}{l}
p \\
j
\end{array}\right) A^{a b}\left(c^{1} a b\right)^{j} \phi^{p-j+1} \\
& +\sum_{j=1}^{p}\left(\begin{array}{l}
p \\
j
\end{array}\right)\left\{B\left(\Gamma^{j}\right)-A^{a b}\left(c^{0}{ }_{a b}\right)^{j} \partial_{\tau}-A^{a b}\left(c^{+}{ }_{a b}\right)^{j} X_{+}\right. \\
& \left.-A^{a b}\left(c^{-}{ }_{a b}\right)^{j} X_{-}\right\} \phi^{p-j} .
\end{aligned}
$$


We note that the form of the operator $\mathcal{A}_{p}$ is independent of the solution. In the case where the Bianchi equation is considered as linear equation on Minkowski space the functions $R^{p}$ vanish for all $p$.

We shall also use the constraint equations (5.49). They imply conditions

$$
\mathcal{B}_{p} \phi^{p}=S^{p}, \quad p=2,3, \ldots
$$

with a linear differential operator, given by

$$
\mathcal{B}_{p} \phi^{p}=\left\{-\tau F^{01} \partial_{\tau}+p F^{01}+\frac{1}{2}\left(F^{00} X_{+}-F^{11} X_{-}\right)\right\} \phi^{p}
$$

and a right-hand side, given by

$$
\begin{aligned}
\sqrt{2} S^{p}= & -\sum_{j=2}^{p}\left(\begin{array}{c}
p \\
j
\end{array}\right) F^{a b}\left(c^{1}{ }_{a b}\right)^{j} \phi^{p-i+1} \\
+ & +\sum_{j=1}^{p}\left(\begin{array}{l}
p \\
j
\end{array}\right)\left\{H\left(\Gamma^{j}\right)-F^{a b}\left(c^{0}{ }_{a b}\right)^{j} \partial_{\tau}-F^{a b}\left(c^{+}{ }_{a b}\right)^{j} X_{+}\right. \\
& \left.-F^{a b}\left(c^{-}{ }_{a b}\right)^{j} X_{-}\right\} \phi^{p-j} .
\end{aligned}
$$

Again we note that the form of the operator $\mathcal{B}_{p}$ is independent of the solution.

It follows that given $v^{0}, \ldots, v^{p-1}, \phi^{0}, \ldots, \phi^{p-1}$ for some $p \geq 1$, the linear ordinary differential equation (8.2) allows to solve for $v^{p}$ and after this the linear symmetric hyperbolic system (8.3) determines $\phi^{p}$ if the data (5.57)-(5.56) are assumed.

\section{Lemma 8.5.}

(i) The functions $\left(c_{a b}^{1}-\rho x_{a b}\right)^{p}, v^{p}, \phi^{p}, p=1,2, \ldots$, on I are of expansion type $p-2$, $p-1, p$, respectively.

(ii) The functions $R^{p}, S^{p}$ are of expansion type $p-1$ for $p=1,2, \ldots$

(iii) If for a given integer $p \geq 1$ the data for $\phi^{p}$ on $C_{a, x}$ are of type $p-1$, then the function $\phi^{p}$ on $I$ is of type $p-1$.

By the explicit form of the initial data and by Lemma 8.4, assertion (i) is consistent with the structure of the intial data. That the statements (i) are true for $p=1$ follows from (7.2)-(7.12). Next we observe that $\left(c^{\rho} a b-\rho x_{a b}\right)^{p}=0, p=0,1$, and that Eq. (5.40) together with (7.2) and (7.3) imply equations

$$
\partial_{\tau}\left(c^{1}{ }_{a b}-\rho x_{a b}\right)^{q}=-\sum_{j=1}^{q-1}\left(\begin{array}{l}
q \\
j
\end{array}\right) \chi_{(a b)}^{j}{ }^{e f}\left(c^{1}{ }_{e f}-\rho x_{e f}\right)^{q-j}-q \chi_{(a b)}^{q-1}{ }^{f f} x_{e f}
$$

on $I$ for $q=0,1, \ldots$ Let $k \geq 1$ be an integer and assume as induction hypothesis assertions (i) for $p=1,2, \ldots, k$. Eq. (8.9) implies then that $\partial_{\tau}\left(c^{1} a b-\rho x_{a b}\right)^{k+1}$ is of expansion type $k-1$. The structure of the initial data implies that $\left(c^{1}{ }_{a b}-\rho x_{a b}\right)^{k+1}$ is of expansion type $k-1$. By Lemma $8.3 L^{j}$ has expansion type $j-1$. Eqs. (8.2) together with the induction 
hypothesis therefore allows to conclude that $v^{k+1}$ is of expansion type $k$. Finally, observing that $\left(c^{1}{ }_{a b}-\rho x_{a b}\right)^{q}=\left(c^{\rho}{ }_{a b}\right)^{q}$ for $q \geq 2$, the induction hypothesis implies that $R^{k+1}$ is of type $k$. Eqs. (8.3) and the structure of the data then allow to conclude that $\phi^{k+1}$ is of type $k+1$. From the given arguments (ii), (iii) follow now immediately.

To see the consequences of the degeneracy of the matrix (7.6) we analyse the system of transport equations in more detail. Eqs. (8.3) read explicitly

$$
\begin{aligned}
& (1+\tau) \partial_{\tau} \phi_{0}^{p}+X_{+} \phi_{1}^{p}-(p-2) \phi_{0}^{p}=R_{0}^{p}, \\
& \partial_{\tau} \phi_{1}^{p}+\frac{1}{2}\left(X_{+} \phi_{2}^{p}+X_{-} \phi_{0}^{p}\right)+\phi_{1}^{p}=R_{1}^{p}, \\
& \partial_{\tau} \phi_{2}^{p}+\frac{1}{2}\left(X_{+} \phi_{3}^{p}+X_{-} \phi_{1}^{p}\right)=R_{2}^{p}, \\
& \partial_{\tau} \phi_{3}^{p}+\frac{1}{2}\left(X_{+} \phi_{4}^{p}+X_{-} \phi_{2}^{p}\right)-\phi_{3}^{p}=R_{3}^{p}, \\
& (1-\tau) \partial_{\tau} \phi_{4}^{p}+X_{-} \phi_{3}^{p}+(p-2) \phi_{4}^{p}=R_{4}^{p},
\end{aligned}
$$

while Eqs. (8.6) read

$$
\begin{aligned}
\tau \partial_{\tau} \phi_{1}^{p}+\frac{1}{2}\left(X_{+} \phi_{2}^{p}-X_{-} \phi_{0}^{p}\right)-p \phi_{1}^{p} & =S_{1}^{p} \\
\tau \partial_{\tau} \phi_{2}^{p}+\frac{1}{2}\left(X_{+} \phi_{3}^{p}-X_{-} \phi_{1}^{p}\right)-p \phi_{2}^{p} & =S_{2}^{p} \\
\tau \partial_{\tau} \phi_{3}^{p}+\frac{1}{2}\left(X_{+} \phi_{4}^{p}-X_{-} \phi_{2}^{p}\right)-p \phi_{3}^{p} & =S_{3}^{p}
\end{aligned}
$$

The two systems entail

$$
\begin{aligned}
& (p+\tau) \phi_{1}^{p}=\frac{1}{2}\left((1-\tau) X_{+} \phi_{2}^{p}-(1+\tau) X_{-} \phi_{0}^{p}\right)-S_{1}^{p}+\tau R_{1}^{p}, \\
& p \phi_{2}^{p}=\frac{1}{2}\left((1-\tau) X_{+} \phi_{3}^{p}-(1+\tau) X_{-} \phi_{1}^{p}\right)-S_{2}^{p}+\tau R_{2}^{p}, \\
& (p-\tau) \phi_{3}^{p}=\frac{1}{2}\left((1-\tau) X_{+} \phi_{4}^{p}-(1+\tau) X_{-} \phi_{2}^{p}\right)-S_{3}^{p}+\tau R_{3}^{p},
\end{aligned}
$$

from which we get

$$
\begin{aligned}
& 4 p(p+\tau) \phi_{1}^{p}+\left(1-\tau^{2}\right) X_{+} X_{-} \phi_{1}^{p}-(1-\tau)^{2} X_{+}^{2} \phi_{3}^{p}+2 p(1+\tau) X_{-} \phi_{0}^{p} \\
& =-2(1-\tau) X_{+}\left(S_{2}^{p}-\tau R_{2}^{p}\right)-4 p\left(S_{1}^{p}-\tau R_{1}^{p}\right), \\
& 4 p(p-\tau) \phi_{3}^{p}+\left(1-\tau^{2}\right) X_{-} X_{+} \phi_{3}^{p}-(1+\tau)^{2} X_{-}^{2} \phi_{1}^{p}-2 p(1-\tau) X_{+} \phi_{4}^{p} \\
& =2(1+\tau) X_{-}\left(S_{2}^{p}-\tau R_{2}^{p}\right)-4 p\left(S_{3}^{p}-\tau R_{3}^{p}\right) \text {. }
\end{aligned}
$$

Observing Theorem 4.1, we expand now

$$
\phi_{j}^{p}=\sum_{q=|2-j|}^{p} \sum_{k=0}^{2 q} a_{j, p ; q, k} T_{2 q}{ }^{k}{ }_{q-2+j}
$$

with complex coefficients $a_{j, p ; q, k}$. Notice that this expansion differs from expansions (3.39) and (3.40) by factors $p$ !.

In the case of the system (8.18)-(8.20) expansion (8.23) leads to an equation of the form 


$$
\begin{aligned}
& \left(\begin{array}{ccc}
p+\tau & -\frac{1-\tau}{2} \beta_{2 q, q} & 0 \\
-\frac{1+\tau}{2} \beta_{2 q, q} & p & -\frac{1-\tau}{2} \beta_{2 q, q+1} \\
0 & -\frac{1+\tau}{2} \beta_{2 q, q+1} & p-\tau
\end{array}\right)\left(\begin{array}{l}
a_{1, p: q, k} \\
a_{2, p: q, k} \\
a_{3, p ; q, k}
\end{array}\right) \\
& =\left(\begin{array}{l}
A_{1, p: q, k} \\
A_{2, p: q, k} \\
A_{3, p ; q, k}
\end{array}\right) .
\end{aligned}
$$

where for given values of the indices $p, q, k$ with $p \geq 0,|2-j| \leq q \leq p .0 \leq k \leq 2 q$. the quantities $A_{j, p ; q, k}, j=1,2,3$, are functionals of the coefficients $a_{j, p^{\prime}: q^{\prime}, k^{\prime}}, 0 \leq j \leq 4$, $p^{\prime}<p$, and $a_{0, p: q, k}, a_{4, p: q, k}$. The determinant of the matrix on the left-hand side, given by

$$
D \equiv \frac{1}{2} p\left\{2 p^{2}-q(q+1)+\tau^{2}(q(q+1)-2)\right\},
$$

satisfies $D \geq 2$ for $p \geq 2,0 \leq q \leq p,|\tau| \leq 1$. For $p \geq 2 \mathrm{Eq}$. (8.24) can therefore be solved for the expansion coefficients $a_{1, p ; q, k}, a_{2, p ; q, k}, a_{2, p ; q, k}$. This allows us to reduce the problem of solving the system (8.10)-(8.14) for five unknowns to solving a system for two unknowns.

Assuming the values of indices $p, q, k$ of $a_{j, p ; q . k}$ as fixed, we shall suppress them in the following equations. Using Eqs. (3.35) we obtain from Eqs. (8.21) and (8.22) the algebraic conditions

$$
\begin{aligned}
& \left\{4 p(p+\tau)-\left(1-\tau^{2}\right) \beta_{2 q . q}^{2}\right\} a_{1}-(1-\tau)^{2} \beta_{2 q . q+1} \beta_{2 q . q} a_{3} \\
& \quad=2 p(1+\tau) \beta_{2 q, q-1} a_{0}+U_{1}, \\
& \left\{4 p(p-\tau)-\left(1-\tau^{2}\right) \beta_{2 q \cdot q+1}^{2}\right\} a_{3}-(1+\tau)^{2} \beta_{2 q, q} \beta_{2 q \cdot q+1} a_{1} \\
& \quad=2 p(1-\tau) \beta_{2 q, q+2} a_{4}+U_{3} .
\end{aligned}
$$

We define for $p \geq 2$ functions

$$
\begin{aligned}
f(\tau)= & 2(p+1)(p-1)-(q-1)(q+2)\left(1-\tau^{2}\right), \\
g(\tau)= & -(p-2) f(\tau)+(q-1)(q+2) \\
& \times\left((p+1)(1+\tau)-(1+\tau)^{2}-\frac{q(q+1)}{4 p}(1-\tau)(1+\tau)^{2}\right) . \\
h(\tau)= & \frac{(q-1) q(q+1)(q+2)}{4 p}(1-\tau)^{3},
\end{aligned}
$$

such that

$$
f \geq 1 \text { for } p \geq 2, \quad 0 \leq q \leq p,|\tau| \leq 1 .
$$

For a given function $l=l(\tau)$ we defined the function $l_{s}$ by $l_{s}(\tau)=l(-\tau)$. From the equations above we get

$$
f a_{1}=\left((p+1)(1+\tau)-(1+\tau)^{2}-\frac{(1-\tau)(1+\tau)^{2}}{4 p} \beta_{2 q, q+1}^{2}\right) \beta_{2 q, q-1} a_{0}
$$




$$
\begin{aligned}
& +\frac{(1-\tau)^{3}}{4 p} \beta_{2 q, q} \beta_{2 q, q+1} \beta_{2 q, q+2} a_{4}+\hat{U}_{1}, \\
f a_{3}= & \left((p+1)(1-\tau)-(1-\tau)^{2}-\frac{(1-\tau)^{2}(1+\tau)}{4 p} \beta_{2 q, q}^{2}\right) \beta_{2 q, q+2} a_{4} \\
& +\frac{(1+\tau)^{3}}{4 p} \beta_{2 q, q-1} \beta_{2 q, q} \beta_{2 q, q+1} a_{0}+\hat{U}_{3} .
\end{aligned}
$$

Rewriting Eqs. (8.10) and (8.14) in terms of the expansion coefficients and using Eqs. (3.35), we get

$$
\begin{aligned}
& (1+\tau) f a_{0}^{\prime}+g a_{0}+h a_{4}=T_{0}, \\
& -(1-\tau) f_{s} a_{4}^{\prime}+g_{s} a_{4}+h_{s} a_{0}=T_{4},
\end{aligned}
$$

where the prime denotes the derivative with respect to $\tau$.

It is important here to observe that the quantities $U_{1}, U_{3}, \hat{U}_{1}, \hat{U}_{3}, T_{0}, T_{4}$ depend for $0 \leq q \leq p-1$ on the coefficients $a_{j^{\prime}, p^{\prime} ; q^{\prime}, k^{\prime}}$ with $p^{\prime}<p$ and that they vanish identically for $q=p$.

We see that Eqs. (8.18)-(8.20) allow to express the coefficients $a_{1}, a_{2}, a_{3}$ in terms of $a_{0}$, $a_{4}$ and lower order terms. Thus we find that the problem of solving Eqs. (8.10)-(8.14) is reduced to solving the system (8.25) and (8.26). The remaining components of the solution are determined by algebraic operations which do not lead to singular terms. Of course in each order we have to solve the equations arising from (5.40)-(5.45).

Lemma 8.6. For the homogeneous system arising from (8.25) and (8.26) by setting $T_{0}=0$, $T_{4}=0$ the following holds true:

(i) If $0 \leq q \leq p-1$, the system has an analytic fundamental matrix on $|\tau|<1$ which exterds analytically through $\tau= \pm 1$.

(ii) If $q=p$, the solutions satisfying $a_{0}(0)=a_{4}(0)$ extend analytically through $\tau= \pm 1$ while any other solution has logarithmic singularities at $\tau= \pm 1$.

(iii) For $0 \leq q \leq p$ the Wronskian of any fundamental matrix is of the form $w=c f(\tau)(1-$ $\left.\tau^{2}\right)^{p-2}$ with some constant $c \neq 0$.

If the $2 \times 2$ matrix $X(\tau)$ is a fundamental matrix of the system, the Wronskian $w=\operatorname{det}(X)$ satisfies $w^{\prime}=k(\tau) w$ with

$$
k(\tau)=-\frac{g(\tau)}{(1 \mid \tau) f(\tau)}+\frac{g(-\tau)}{(1-\tau) f(-\tau)}=\frac{p-2}{1+\tau}-\frac{p-2}{1-\tau}+\frac{f^{\prime}(\tau)}{f(\tau)}
$$

This implies $w=(w(0) / f(0)) f(\tau)\left(1-\tau^{2}\right)^{p-2}$, which shows (iii).

For $q=0,1$ we have $h=0$ and Eqs. (8.25) and (8.26) decouple. The general solution is given in the case $q=0$ by $a_{0}=c_{0}(1+\tau)^{p-2}(p+\tau), a_{4}=c_{4}(1-\tau)^{p-2}(p-\tau)$, and in the case $q=1$ by $a_{0}=c_{0}^{\prime}(1+\tau)^{p-2}, a_{4}=c_{4}^{\prime}(1-\tau)^{p-2}$, with constant coefficients $c_{0}$, $c_{4}, c_{0}^{\prime}, c_{4}^{\prime}$. 
For $q \geq 2$ we study second order equations satisfied by $a_{0}, a_{4}$. Considering $T_{0}, T_{4}$ in Eqs. (8.25) and (8.26) for a moment as name for the quantities on the left-hand sides, we find

$$
\begin{aligned}
& (1-\tau) h f T_{0}^{\prime}+h^{2} T_{4}-\left\{(1-\tau) f h^{\prime}+h g_{s}\right\} T_{0} \\
& \quad=h f^{2}\left\{\left(1-\tau^{2}\right) a_{0}^{\prime \prime}+\{4+2(p-1) \tau\} a_{0}^{\prime}+(q+p)(q-p+1) a_{0}\right\} \\
& (1+\tau) h_{s} f T_{4}^{\prime}-h_{s}^{2} T_{0}-\left\{(1+\tau) f h_{s}^{\prime}+h_{s} g\right\} T_{4} \\
& \quad=-h_{s} f^{2}\left\{\left(1-\tau^{2}\right) a_{4}^{\prime \prime}+\{-4+2(p-1) \tau\} a_{4}^{\prime}+(q+p)(q-p+1) a_{4}\right\} .
\end{aligned}
$$

Thus, for $2 \leq p, 2 \leq q \leq p$, the coefficients $a_{0}, a_{4}$ satisfy

$$
\begin{aligned}
& \left(1-\tau^{2}\right) a_{0}^{\prime \prime}+\{4+2(p-1) \tau\} a_{0}^{\prime}+(q+p)(q-p+1) a_{0}=0, \\
& \left(1-\tau^{2}\right) a_{4}^{\prime \prime}+\{-4+2(p-1) \tau\} a_{4}^{\prime}+(q+p)(q-p+1) a_{4}=0 .
\end{aligned}
$$

i.e. Jacobi equations

$$
\begin{aligned}
0 & =D_{(n . \alpha . \beta)} a \\
& \equiv\left(1-\tau^{2}\right) a^{\prime \prime}+\{\beta-\alpha-(\alpha+\beta+2) \tau\} a^{\prime}+n(n+\alpha+\beta+1) a,
\end{aligned}
$$

where

$$
\begin{aligned}
& \alpha=-p-2, \quad \beta=-p+2 \text { and } \\
& n=n_{1}=p+q \quad \text { or } \quad n=n_{2}=p-q-1,
\end{aligned}
$$

in the case of (8.27) and the values of $\alpha$ and $\beta$ are swapped in the case of (8.28). Eq. (8.29) is well known from the theory of Jacobi polynomials.

We note that $a_{0}$ solves (8.27) if and only if $a_{0 s}$ solves (8.28). Thus it will be sufficient to study Eq. (8.27).

In the following discussion of the solutions to (8.29) for the values $(8.30)$ of the parameters we shall make use and quote some of the results of [23] without referring to this monograph in each case.

For integers $n \geq 0$ and arbitrary complex parameters $\alpha, \beta$ a class of solutions to (8.29) is given by the generalized Jacobi polynomials which are of the form

$$
P_{n}^{(\alpha, \beta)}(\tau)=\frac{1}{n !} \sum_{i=0}^{n} c_{\nu}\left(\frac{\tau-1}{2}\right)^{v}
$$

with coefficients

$$
\begin{aligned}
c_{0}= & (\alpha+1)(\alpha+2) \cdots(\alpha+n), \\
c_{v}= & \frac{n !}{\nu !(n-v) !}(\alpha+v+1)(\alpha+v+2) \\
& \times \cdots(\alpha+n)(n+1+\alpha+\beta)(n+2+\alpha+\beta) \cdots(n+v+\alpha+\beta)
\end{aligned}
$$

for $1 \leq v \leq n-1$,

$$
c_{n}=(n+1+\alpha+\beta)(n+2+\alpha+\beta) \cdots(2 n+\alpha+\beta) .
$$


They satisfy $P_{0}^{(\alpha, \beta)}(\tau) \equiv 1$ and $P_{n}^{(\alpha, \beta)}(-\tau)=(-1)^{n} P_{n}^{(\beta, \alpha)}(\tau)$.

From the coefficients above it follows that the polynomials $P_{n_{1}}^{(-p-2,-p+2)}$ vanish identically while the polynomials $Q_{2}=P_{n_{2}}^{(-p-2,-p+2)}, p \geq 3,2 \leq q \leq p-1$ are of degree $n_{2}$.

To find further non-trivial solutions we use the identities

$$
\begin{aligned}
& D_{(n, \alpha, \beta)}\left(\left(\frac{1-\tau}{2}\right)^{-\alpha} a(\tau)\right)=\left(\frac{1-\tau}{2}\right)^{-\alpha} D_{(n+\alpha,-\alpha, \beta)} a(\tau), \\
& D_{(n, \alpha, \beta)}\left(\left(\frac{1+\tau}{2}\right)^{-\beta} a(\tau)\right)=\left(\frac{1+\tau}{2}\right)^{-\beta} D_{(n+\beta, \alpha,-\beta)} a(\tau), \\
& D_{(n, \alpha, \beta)}\left(\left(\frac{1-\tau}{2}\right)^{-\alpha}\left(\frac{1+\tau}{2}\right)^{-\beta} a(\tau)\right) \\
& =\left(\frac{1-\tau}{2}\right)^{-\alpha}\left(\frac{1+\tau}{2}\right)^{-\beta} D_{(n+\alpha+\beta,-\alpha,-\beta)} a(\tau),
\end{aligned}
$$

which hold for $|\tau|<1$, arbitrary $C^{2}$-functions $a$, and arbitrary values of the parameters $\alpha$, $\beta, n$.

Using (8.31) with $n=n_{1}, \alpha=-2-p, \beta=-p+2$ we find the polynomial solutions

$$
Q_{1}=\left(\frac{1-\tau}{2}\right)^{p+2} P_{q-2}^{(p+2 .-p+2)}(\tau), \quad p \geq 2, \quad 2 \leq q \leq p,
$$

of degree $n_{1}$. Since $n_{2}<n_{1}$ the solutions $Q_{1}, Q_{2}$ are independent of each other and define a fundamental system of Eq. (8.27) for $p \geq 3,2 \leq q \leq p-1$.

For later use we note that by using (8.32) with $n=n_{1}, \alpha=-2-p, \beta=-p+2$ we obtain the solution

$$
Q_{3}=\left(\frac{1+\tau}{2}\right)^{p-2} P_{q+2}^{(-p-2 . p-2)}(\tau)
$$

which is a polynomial of degree $n_{1}$. For $p \geq 3,2 \leq q \leq p-1$ it can be expanded in terms of the solutions considered above with coefficients which are determined by evaluating at the points $\tau= \pm 1$. We get

$$
\begin{aligned}
& \left(\frac{1+\tau}{2}\right)^{p-2} P_{q+2}^{(-p-2, p-2)} \\
& =(-1)^{p}\left(\left(\frac{1-\tau}{2}\right)^{p+2} P_{q-2}^{(p+2,-p+2)}-P_{n_{2}}^{(-p-2,-p+2)}\right) .
\end{aligned}
$$

Having found the general solutions to (8.27) and (8.28) for $q \leq p-1$, we determine the general solution to (8.25) and (8.26). It has been given already in the case $p \geq 2, q=0,1$. We consider the case $p \geq 3,2 \leq q \leq p-1$.

We write solutions to Eqs. (8.25) and (8.26) as column vectors $u_{1}=\left(\begin{array}{l}a_{0} \\ a_{4}\end{array}\right)$. There exists a fundamental matrix of the system (8.25) and (8.26) with $T_{0}=0, T_{4}=0$ which has column vectors 


$$
u_{1}=\left(\begin{array}{c}
c_{1}^{11} Q_{1}+c_{1}^{12} Q_{2} \\
c_{1}^{21} Q_{1 s}+c_{1}^{22} Q_{2 s}
\end{array}\right), \quad u_{2}=\left(\begin{array}{c}
c_{2}^{11} Q_{1}+c_{2}^{12} Q_{2} \\
c_{2}^{21} Q_{1 s}+c_{2}^{22} Q_{2 s}
\end{array}\right)
$$

with real coeffcients $c_{i}^{j k}$. By taking suitable linear combinations of these solutions if necessary, we can assume that $c_{2}^{11}=0, c_{2}^{12}=1$. Observing that $n_{2}<n_{1}$, we conclude from (8.25) that $c_{2}^{21} Q_{1 s}+c_{2}^{22} Q_{2 s}$ must be a polynomial of degree $n_{2}$ and thus $c_{2}^{21}=0$. Adding a suitable multible of $u_{2}$ to $u_{1}$ and normalizing we find a fundamental matrix with column vectors

$$
u_{1}=\left(\begin{array}{c}
Q_{1} \\
y Q_{1 s}+z Q_{2 s}
\end{array}\right), \quad u_{2}=\left(\begin{array}{c}
Q_{2} \\
x Q_{2 s}
\end{array}\right)
$$

with real numbers $x, y, z$ such that $x \neq 0, y \neq 0$.

To determine the coefficients we insert $u_{1}$ and $u_{2}$ into (8.25) and (8.26) and evaluate at $\tau= \pm 1$. Using the values

$$
\begin{aligned}
& Q_{1}(1)=0, \quad Q_{1}(-1)=Q_{2}(-1)=\frac{(p-3) !}{(q-2) ! n_{2} !}, \\
& Q_{2}(1)=(-1)^{n_{2}} \frac{(p+1) !}{(q+2) ! n_{2} !},
\end{aligned}
$$

we get $x=z=-y=(-1)^{n_{2}}$. Taking linear combinations of the solutions so obtained and observing (8.34) we get a fundamental matrix

$$
X=\left(\begin{array}{cc}
Q_{1} & (-1)^{q} Q_{3} \\
(-1)^{q} Q_{3 s} & Q_{1 s}
\end{array}\right) .
$$

This ends the proof of assertion (i) of Lemma 8.6.

We write $a_{0}=((1-\tau) / 2)^{p+2}((1+\tau) / 2)^{p-2} a(\tau)$ to discuss the solutions of $(8.27)$ for $p \geq 2, q=p$. Using (8.33) with $n=n_{1}, \alpha=-p-2, \beta=-p+2$ we rewrite (8.27) in the form

$$
\begin{aligned}
& D_{\left(n_{1},-p-2,-p+2\right)}\left(\left(\frac{1-\tau}{2}\right)^{p+2}\left(\frac{1+\tau}{2}\right)^{p-2} a(\tau)\right) \\
& =\left(\frac{1-\tau}{2}\right)^{p+2}\left(\frac{1+\tau}{2}\right)^{p-2} D_{(0, p+2, p-2)} a(\tau)=0 .
\end{aligned}
$$

The equation $D_{(0, p+2, p-2)} a=0$ can be solved explicitly. We get

$$
a_{0}=\left(\frac{1-\tau}{2}\right)^{p+2}\left(\frac{1+\tau}{2}\right)^{p-2}\left(d_{0}+d_{1} \int_{0}^{\tau} \frac{\mathrm{d} \sigma}{(1+\sigma)^{p-1}(1-\sigma)^{p+3}}\right)
$$

with arbitrary coefficients $d_{0}, d_{1}$. Notice that for $d_{1} \neq 0$ these solutions have logarithmic singularities at $\tau= \pm 1$.

We verify by direct calculation that a polynomial solution of (8.25) and (8.26) with $T_{0}=0, T_{4}=0$ is given by 


$$
u=\left(\begin{array}{c}
(1-\tau)^{p+2}(1+\tau)^{p-2} \\
(1+\tau)^{p+2}(1-\tau)^{p-2}
\end{array}\right)
$$

It follows that any solution with $a_{0}(0) \neq a_{4}(0)$ is not proportional to this one and has logarithmic singularities at $\tau= \pm 1$. This proves (ii).

We can now prove Theorem 8.2. For given $p, q$ we write Eqs. (8.25) and (8.26) in the form $y^{\prime}=A(\tau) y+b$ with $b$ the column vector defined by $T_{0}, T_{4}$, and assume that $X(\tau)$ is a fundamental matrix of the associated homogeneous system, i.e. $X^{\prime}=A(\tau) X, X(0)=X_{0}$, $\operatorname{det}\left(X_{0}\right) \neq 0$. The solution to the inhomogeneous problem with $y\left(\tau_{0}\right)=y_{0}$ is given by

$$
y(\tau)=X(\tau)\left(X_{0}^{-1} y_{0}+\int_{0}^{\tau} X(\sigma)^{-1} b(\sigma) \mathrm{d} \sigma\right) .
$$

The considerations which led to Lemma 8.5 show that for $q=p$ we have $b \equiv 0$ whence $y(\tau)=X(\tau) X_{0}^{-1} y_{0}$. From Theorem 4.1 and from the discussion above it follows that this solution is smooth through $I^{ \pm}$if and only if condition (4.7) is satisfied.

\section{Concluding remarks}

We have obtained in this article a setting which enables us for the first time to discuss in detail the consequences of the conformal Einstein evolution near space-like infinity. The analysis has shown that, in a sense, we can gain complete control on the solutions to the transport equations along the total characteristic at space-like infinity. These equations allow us to relate the behaviour of solutions to Einstein's vacuum field equation near null infinity to properties of the free initial data.

The following problems would have to be solved for a complete understanding of the situation. The solutions to the transport equations should be further investigated to see whether condition (4.7) is sufficient for the regularity of their solutions or if more restrictive regularity conditions need to be imposed. Given a reasonable set of regularity conditions, the existence of solutions to the problem formulated in Section 5 should be shown and the continuous dependence of the solution on the data should be discussed.

This proof would not only be of interest for theoretical reasons. If in the complete analysis the equations turn out to be as good-natured as suggested by the discussion in this article, they should be of enormous practical use in the numerical construction of space-times.

The discussion should be extended to include non-trivial second fundamental forms. In this case certainly further regularity requirements will arise. Following the argument which led in [10] to condition (4.7), we would arrive at generalized regularity conditions in the following way. Observing (2.38), (2.36), and (2.34), where we can assume for the following purpose that $\hat{\nabla}=\nabla$, we get

$$
\Omega^{2} d_{i j}=D_{i} D_{j} \Omega+\Omega f_{i j}-\bar{s} h_{i j}
$$

where we set 


$$
\begin{aligned}
& \bar{s}=\frac{1}{3} D_{k} D^{k} \Omega+\Omega h^{j k} A_{j k}, \\
& f_{j k}=l_{j k}+\chi_{l j} \chi_{k}{ }^{l}-\chi_{l}^{l} \chi_{j k}+\frac{1}{12}\left\{\left(\chi_{l}{ }^{l}\right)^{2}-\chi_{i l} \chi^{i l}\right\} h_{j k}
\end{aligned}
$$

and all indices are assumed to take values 1,2,3. With (2.34) and the assumption $\Sigma=0$. Eq. (3.10) of [12] can be written

$$
D_{j} \bar{s}=-A_{j k} D^{k} \Omega \text {. }
$$

Applying $D_{k}$ to both sides of (9.1), antisymmetrizing in the indices $i$ and $k$, contracting with $D^{j} \Omega$, and using the equation above as well as (2.38), (2.36), and (2.34) to simplify, we get

$$
D_{[k} f_{i \mid j} D^{j} \Omega=D_{[k} \Omega d_{i] j} D^{j} \Omega+\Omega D_{[k} d_{i] j} D^{j} \Omega .
$$

This entails

$$
B_{j k} D^{j} \Omega D^{k} \Omega=\Omega D_{k} d_{i j} \epsilon^{k i}{ }_{l} D^{l} \Omega D^{j} \Omega .
$$

where we set $B_{k l}=D_{i} f_{j(k} \epsilon^{i j}{ }_{l)}$. Setting furthermore $C_{k l}=D_{i} \chi_{j(k} \epsilon^{i j}{ }_{l)}$, Eq. (2.39) reads

$$
C_{k l}=-\Omega d_{k l}^{*} \text {. }
$$

We note here that the symmetric trace-free tensor fields $B_{k l}, C_{k l}$ have been used by R. Beig and L. Szabados to characterize initial data for conformally flat solutions. ${ }^{2}$ Making now as in [10] the assumption that the fields $\Omega, h_{i j}, \chi_{i j}, d_{i j}, d_{i j}^{*}$, extend to real analytic fields at the point $i$ of our initial hypersurface $S$, we obtain for the complex analytic extensions of the fields $B_{k l}, C_{k l}$ the following generalization of condition (ii) of Theorem 4.2

$$
B_{j k} D^{j} \Omega D^{k} \Omega=0, \quad C_{k l}=0 \quad \text { on } N .
$$

In terms of the space-spinor representations of $B_{k l}, C_{k l}$ these equations translate into the generalization

$$
\begin{aligned}
D_{\left(a_{q} b_{q}\right.} & \cdots D_{a_{1} b_{1}} B_{a b c d)}(i)=0, \quad D_{\left(a_{p} b_{p}\right.} \cdots D_{\left.a_{1} b_{1}\right)} C_{a b c d}(i)=0, \\
q . p & =0,1,2, \ldots
\end{aligned}
$$

of (4.7).

Of course, in the case of non-vanishing ADM-mass one would have to go through the analogue of the analysis performed in Sections 2, 4, 7 and 8, before the relevance of these condition for the initial value problem can be properly assessed. Furthermore, one would like to understand whether the conditions above can be expressed in terms of the data which can be given freely in the construction of constrained initial data.

These questions, as well as why our setting seems to work so well and what the geometrical meaning of the regularity conditions could be, will be pursued elsewhere.

\footnotetext{
${ }^{2}$ I am grateful to L. Szabados for a discussion on this point.
} 


\section{Definitions and general identities}

The following quantities are used in many calculations.

$$
\begin{aligned}
& x_{a b}=\sqrt{2} \epsilon_{(a}{ }^{0} \epsilon_{b)}{ }^{1}, \quad y_{a b} \equiv-\frac{1}{\sqrt{2}} \epsilon_{a}{ }^{1} \epsilon_{b}{ }^{1}, \quad z_{a b} \equiv \frac{1}{\sqrt{2}} \epsilon_{a}{ }^{0} \epsilon_{b}{ }^{0}, \\
& \epsilon_{a b c d}^{k} \equiv \epsilon_{(a}{ }^{(e} \epsilon_{b}{ }^{f} \epsilon_{c}{ }^{g} \epsilon_{d)}{ }^{h)_{k}}, \quad \gamma^{*}{ }_{a b c d} \equiv \frac{1}{2}\left(\epsilon_{a c} x_{b d}+\epsilon_{b d} x_{a c}\right) .
\end{aligned}
$$

They satisfy the following relations:

$$
\begin{aligned}
& x_{a b} x^{a b}=-1, \quad x_{a b} y^{a b}=x_{a b} z^{a b}=0 \text {, } \\
& y_{a b} y^{a b}=0, \quad y_{a b} z^{a b}=-\frac{1}{2}, \quad z_{a b} z^{a b}=0 \text {, } \\
& y_{a} f_{x_{b f}}=\frac{1}{\sqrt{2}} y_{a b}, \quad z_{a}{ }^{f} x_{b f}=-\frac{1}{\sqrt{2}} z_{a b}, \\
& h_{a b(c}{ }^{f} x_{d) f}=\frac{1}{2}\left(\epsilon_{a c} x_{b d}+\epsilon_{b d} x_{a c}\right) \text {, } \\
& \left(\epsilon_{a b c d}^{k}\right)^{+}=(-1)^{k} \epsilon_{a b c d}^{4-k}, \quad \epsilon_{(a b c d) ;}^{k}=\delta_{j}^{k}\left(\begin{array}{l}
4 \\
k
\end{array}\right)^{-1}, \\
& \epsilon^{2} a b c d \epsilon^{2 a b c d}=\frac{1}{6}, \quad \epsilon^{2} a b{ }^{e f} \epsilon^{2} \text { def }=-\frac{1}{6} \epsilon^{2} a b c d+\frac{1}{18} h_{a b c d}, \\
& \epsilon_{a b c d}^{1} x^{c d}=-\frac{1}{2} z_{a b}, \quad \epsilon_{a b c d}^{2} x^{c d}=-\frac{1}{3} x_{a b}, \quad \epsilon_{a b c d}^{3} x^{c d}=\frac{1}{2} y_{a b}, \\
& \epsilon_{a b c d}^{1} y^{c d}=-\frac{1}{4} x_{a b}, \quad \epsilon_{a b c d}^{2} y^{c d}=\frac{1}{6} y_{a b}, \quad \epsilon_{a b c d}^{3} y^{c d}=0 \\
& \epsilon_{a b c d}^{1} z^{c d}=0, \quad \epsilon_{a b c d}^{2} z^{c d}=\frac{1}{6} z_{a b}, \quad \epsilon_{a b c d}^{3} z^{c d}=\frac{1}{4} x_{a b} . \\
& x_{(a} f_{\left.\epsilon^{1}{ }^{1}\right) c d f}=\frac{1}{2 \sqrt{2}} z_{a b} x_{c d}, \quad x_{(a} f_{\left.\epsilon^{3} b\right) c d f}=\frac{1}{2 \sqrt{2}} y_{a b} x_{c d}, \\
& x_{(a}^{f} \epsilon_{b) c d f}^{2}=\frac{1}{12}\left(\epsilon_{a c} x_{b d}+\epsilon_{b d} x_{a c}\right) .
\end{aligned}
$$

\section{References}

[1] L. Bérard Bergery, Scalar curvature and symmetry group, in: Spectra of Riemannian Manifolds, eds. M. Berger et al. (Kagai, Tokyo, 1983).

[2] M. Cantor and D. Brill, The Laplacian on asymptotically flat manifolds and the specification of scalar curvature, Compositio Math. 43 (1981) 317-330.

[3] A. Chaljub-Simon, Decomposition of the space of covariant two-tensors on $\mathbb{R}^{3}$, Gen. Rel. Grav. 14 (1982) 743-749.

[4] A. Chaljub-Simon and Y. Choquet-Bruhat, Global solutions of the Lichnerowicz equation in general relativity on an asymptotically Euclidean manifold, Gen. Rel. Grav. 12 (1980) 175-185.

[5] Y. Choquet-Bruhat and J.W. York, Jr., The Cauchy problem, in: General Relativity and Gravitation, ed. A. Held, Vol. I (Plenum Press, New York, 1980).

[6] D. Christodoulou and N. O'Murchadha, The boost problem in general relativity, Comm. Math. Phys. 80 (1981) 271-300. 
[7] H. Friedrich, On the regular and the asymptotic characteristic initial value problem for Einstein's vacuum field equations, Proc. Roy. Soc. London A 375 (1981) 169-184.

[8] H. Friedrich, The asymptotic characteristic initial value problem for Einstein's vacuum field equations as an initial value problem for a first-order quasilinear symmetric hyperbolic system. Proc. Roy. Soc. London A 378 (1981) 401-421.

[9] H. Friedrich, On purely radiative space-times, Comm. Math. Phys. 103 (1986) 35-65.

[10] H. Friedrich, On static and radiative space-times, Comm. Math. Phys. 119 (1988) 51-73.

[11] H. Friedrich, On the global existence and the asymptotic behaviour of solutions to the Einstein-MaxwellYang-Mills equations, J. Diff. Geom. 34 (1991) 275-345.

[12] H. Friedrich, Einstein equations and conformal structure: Existence of Anti-de Sitter-type space-times, J. Geom. Phys. 17 (1995) 125-184.

[13] H. Friedrich, Einstein equations and conformal structure, in: Geometric Issues in the Foundations of Science, eds. S. Huggett et al. (Oxford University Press, Oxford, 1997).

[14] P.R. Garabedian, Partial Differential Equations (Wiley, New York, 1964).

[15] D. Gilbarg and N.S. Trudinger, Elliptic Partial Differential Equations of Second Order (Springer. Berlin. 1977).

[16] T. Kato, The Cauchy problem for quasi-linear symmetric hyperbolic systems, Arch. Rational Mech. Anal. 58 (1975) 181-205.

[17] K. Kodaira, Complex Manifolds and Deformation of Complex Structure (Springer, Berlin, 1986).

[18] J.M. Lee and T.H. Parker, The Yamabe problem, Bull. Ame. Math. Soc. 17 (1987) 37-91.

[19] R. Penrose. Asymptotic properties of fields and space-time. Phys. Rev. Lett. 10 (1963) 66-68.

[20] R. Penrose, Zero rest-mass fields including gravitation: Asymptotic behaviour, Proc. Roy. Soc. London A 284 (1965) 159-203.

[21] R. Schoen. Conformal deformation of a Riemannian metric to constant scalar curvature, J. Diff. Geom. 20 (1984) 479-495.

[22] R. Schoen and S.-T. Yau, Proof of the positive mass theorem II. Comm. Math. Phys. 79 (1981) 231-260.

123] G. Szegö, Orthogonal Polynomials, 4th Ed., AMS Colloq. Publ., Vol. 23 (AMS, Providence, RI. 1978).

[24] M.E. Taylor. Pseudodifferential Operators and Nonlinear PDE (Birkhäuser, Boston, 1991).

[25] E. Witten, A new proof of the positive energy theorem, Comm. Math. Phys. 80 (1981) 381-402.

[26] J.W. York. Conformally invariant orthogonal decomposition of symmetric tensors on Riemannian manifolds and the initial-value problem of general relativity, J. Math. Phys. 14 (1973) 456-464. 